Check for updates

Cite this: Mater. Adv., 2020, 1,525

Received 18th March 2020

Accepted 30th April 2020

DOI: $10.1039 / \mathrm{d} 0 \mathrm{ma00108b}$

rsc.li/materials-advances

\section{Recent advances in crystalline carbon dots for superior application potential}

\author{
Ayan Pal, (D) ${ }^{a}$ Md Palashuddin Sk (D) ${ }^{b}$ and Arun Chattopadhyay (D) *ac
}

\begin{abstract}
Over the past decade, the field of carbon dots (Cdots) has opened up a completely new area of research in nanoscience and technology because of their extraordinary optical properties and corresponding multifaceted applications. In this review we aim to highlight the green synthesis of Cdots along with recent speculations, demonstrating the origin of their exciting photoluminescence properties. Further, structural engineering, such as surface state modifications, generating crystallinity by heteroatom doping and controlling size, has induced new characteristics in Cdot structures, indispensable for energy conversion and storage applications. This article covers recent findings on generating long-range ordered structures in Cdots and corresponding advancements in energy related applications such as photovoltaic cells, photocatalysis, optoelectronics, batteries and supercapacitors and photothermal energy conversion. Finally, important challenges such as production of crystalline Cdots and future directions of Cdot based research are discussed.
\end{abstract}

\section{Introduction}

Advanced materials, methods and technology may help meet the high demand for advanced optical, electronic and magnetic

\footnotetext{
${ }^{a}$ Department of Chemistry, Indian Institute of Technology Guwahati, Guwahati-781039, Assam, India. E-mail: arun@iitg:ac.in

${ }^{b}$ Department of Chemistry, Aligarh Muslim University, Aligarh 202002, Uttar Pradesh, India

${ }^{c}$ Centre for Nanotechnology, Indian Institute of Technology Guwahati,
} Guwahati-781039, Assam, India property-based devices so desired for a burgeoning global population. In addition to food, potable water, healthcare and communication, meeting the requirements of energy technologically - while keeping the environment reasonably safe - is an important challenge to be met. In this regard, advancements in chemical catalysts are helping to improve the efficiency and ease of conversion of natural resources to useful energy materials. Thus, the primary challenge comes in the form of twin requirements: energy resources and energy utilizing devices where one is a source of energy and the other one is a sink.

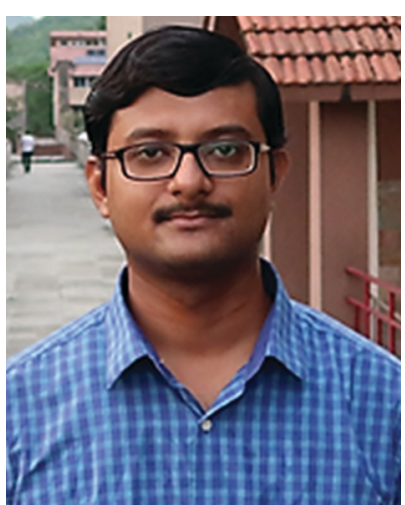

Ayan Pal
Ayan Pal is currently working as a senior research fellow in the Department of Chemistry, Indian Institute of Technology Guwahati, India. He obtained his BSc degree in chemistry from Midnapore College (Vidyasagar University, 2012). Subsequently, he received his master's degree in chemistry from the Indian Institute of Technology Guwahati, Assam, India (2014). His research interest includes synthesis and characterization of carbon-based nanomaterials and their composites for energy storage and conversion applications.

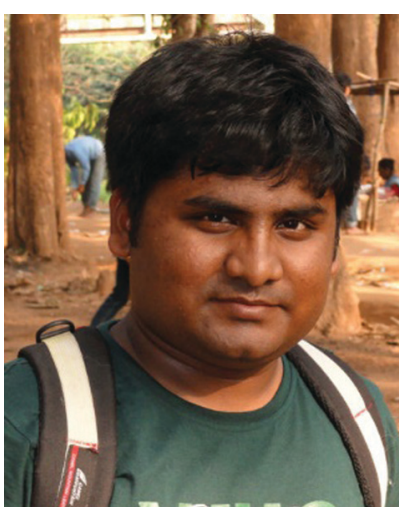

Md Palashuddin Sk
Dr Md Palashuddin Sk has been working as an Assistant Professor in the Department of Chemistry, Aligarh Muslim University, Aligarh, India, since 2015 (November). He received his Master of Science degree (in Chemistry) in 2009 from the Department of Chemistry, Aligarh Muslim University, Aligarh, India. Then, he completed his PhD degree in Chemistry from the Indian Institute of Technology Guwahati, India, under the supervision of Prof. Arun Chattopadhyay in the year of 2015. He also worked as an Associate Project Scientist at the Centre for Nanotechnology, Indian Institute of Technology, Guwahati (2015). His current research interests are primarily focused on the chemistry of luminescent nanomaterials and their applications. 
Both need to be optimized based on advanced scientific developments. In this regard, recent developments in nanoscale science and technology hold promise that may go the beyond laboratory level. That the nanoscale materials exhibit dimensiondependent special properties is particularly encouraging with regard to their application potential in the fields of healthcare, energy and the environment.

Although research on energy resources especially renewable resources is of paramount importance, works on energy efficient devices portend to offer superior options through lowering of energy requirements. In addition, requirement of environmental friendliness provides new opportunities for the advancement of the field. ${ }^{1,2}$ In the context of developing novel and cost-effective devices, utilization of semiconductor quantum dots (QDs), ${ }^{3}$ perovskites, ${ }^{4}$ organic dyes, ${ }^{5}$ and metal or covalent organic frameworks is noteworthy. ${ }^{6,7}$ Recent publications suggest that they offer ease of fabrication in conjunction with energy efficiency. Thus, their application potential in the fields of healthcare, energy and the environment has been greatly demonstrated. However, some of these materials either use resources that are limited or they lack environmental friendliness. On the other hand, natural abundance and environmental friendliness have made carbonaceous nanomaterials an important choice in versatile fields. In addition, the discovery that carbon nanoparticles are present in common food items like bread, corn flakes, biscuits and jaggery, some of which have been consumed by humans for millennia, supports their candidature as safe nanomaterials. Thanks to the unique ability of carbon (C) to form covalent bonds with different hybridization states, there are several nanoscale structures that are supported by carbon. Over the past few decades, several new structures have been identified in the carbon family, e.g., fullerene, carbon nanotubes and graphene and in addition to carbon dots (Cdots), the structures of which are not necessarily well-defined. The application potential of the first three species mentioned above has been extensively demonstrated. On the other hand, that of Cdots is yet to be fully explored. Cdots are luminescent zero-dimensional nanomaterials and have drawn tremendous attention because of their potential use in the fields of biology, ${ }^{8-12}$ sensing, ${ }^{13-16}$

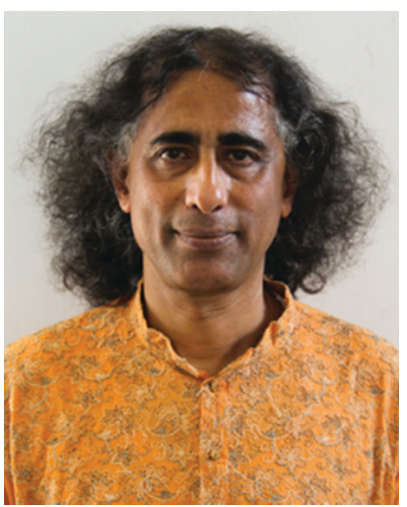

Arun Chattopadhyay
Dr Arun Chattopadhyay is a Professor of Chemistry at the Indian Institute of Technology Guwahati. $\mathrm{He}$ is also associated with the Centre for Nanotechnology, Indian Institute of Technology Guwahati. He obtained his PhD in chemical physics from Columbia University (1992) and completed his postdoctoral research at Stanford University (1995). He is interested, among others, in the programmed assembly of nanoscale particles. catalysis, ${ }^{17-20}$ and energy storage and conversion. ${ }^{21-24}$ Their ease of synthesis, structural flexibility, high photoluminescence quantum yield (PLQY), optical stability and low toxicity have made these nanomaterials superior to other conventional fluorescent materials such as inorganic QDs and organic dyes. It is important mentioning here that of late graphene QDs have been considered as a class of Cdots due to partial similarities in their fundamental properties. ${ }^{20}$ Since their discovery in $2004,{ }^{25}$ although Cdots have been used in the fields of healthcare and sensors, their utilization for energy storage and conversion, and optoelectronic devices has been reported recently (since 2011). The reports suggest that Cdots are not only outstanding optical materials but they are also equally efficient in energy related applications.

Cdots, the synthesis, characterization and applications of which have been reported in the literature, are primarily amorphous in nature. The importance of these Cdots has been well documented. ${ }^{26-31}$ On the other hand, they are far from well-defined materials like bucky balls, carbon nanotubes and graphene. Also, since the structures of Cdots are widely different from each other, it is generally difficult to have a generalized idea about their properties and thus application potential. A part of the problem could be the very amorphous nature of the materials as compared to the other forms of carbon reported above. In addition, since the Cdots are made from materials with complex structures and they have bonding involving heteroatoms, each structure can be unique, depending on the source and the synthesis process. It is no wonder that a general formula representing the common structure of Cdots and thus relating that to their properties may not be possible.

A way out of this uncertainty could be to introduce crystallinity to the Cdot structures. Although this may not lead to one unique structure, the Cdots so generated could have well defined structures. This may help in establishing a better structure-function relationship.

In addition, crystallinity in Cdots may help in their wider application potential as that may improve their redox properties and make electron and energy transfer facile and well-defined. Fortunately, there are several reports indicating the possibility of synthesizing crystalline Cdots, which helps improve their application potential. Importantly, more research work is needed for establishing structure-function relationships of crystalline Cdots and their potential for applications in fabricating devices. ${ }^{20,32-34}$

In this review article, we emphasize the emergence of crystalline Cdots. We start with the already available literature on amorphous Cdots and their application potential. Since synthesis is an important part of generating Cdots with specific properties, we put a stress on that. In addition, we discuss their luminescence and other properties which form the basis of their applications. We then delve into recent reports on generating heteroatom induced non-graphitic long-range ordered structures in Cdots. The potentiality of Cdots being used in organic electronics, such as organic light-emitting devices (OLEDs), electrical sensing, and energy storage and conversion applications is highlighted. The introduction of crystallinity in Cdots 
and the future of such materials for practical applications are also discussed.

\section{Synthesis of Cdots}

Cdots were first discovered at the time of purification of singlewalled carbon nanotubes (SWCNTs) in 2004 and were described as fluorescent carbon impurities by $\mathrm{Xu}$ et $a{ }^{25}$ After that in 2006, quantum-sized nanoscale carbon particles were synthesized using the laser ablation technique by Sun et al. and the obtained particles were termed "carbon dots". ${ }^{35}$ Later in 2007, Liu et al. reported the synthesis of fluorescent "carbon nanoparticles" (size $<2 \mathrm{~nm}$ ) by using a combustion oxidation method. ${ }^{36}$ Since then several physical and chemical methods have been developed to prepare easy-to-synthesize and cost effective Cdots, starting with different precursors. Synthesis of Cdots is categorized into two general approaches: "top-down" and "bottom-up".

In general, a top-down approach of synthesis refers to breaking down or decomposition of a larger entity into smaller components with novel characteristics, whereas a bottom-up approach is described as building-up complex systems starting with small molecular precursors. In the case of Cdots, the top-down approach involves use of larger carbon structures such as activated carbon, carbon nanotubes (CNTs) and graphite by using the arc discharge, ${ }^{25}$ laser ablation, ${ }^{35,37-39}$ ultrasonic wave, ${ }^{40}$ and chemical and electrochemical oxidation techniques. ${ }^{41-45}$ The bottom-up approach, on the other hand, corresponds to the synthesis of Cdots from small molecular precursors through microwave treatment, ${ }^{46-48}$ hydrothermal synthesis, ${ }^{49-53}$ heating, ${ }^{12,16,54,55}$ and plasma treatment. ${ }^{56-58}$ Fig. 1 depicts different synthesis methods for Cdots.

\subsection{Synthesis of Cdots using the top-down approach}

First, Xu et al. introduced synthesis of Cdots using an arc discharge method of single-walled carbon nanotubes (SWCNTs). ${ }^{25}$ During the course of the purification of SWCNTs, two different kinds of impurities were isolated. One of the components was short "tubular carbon" and the other component was fluorescent carbonaceous nanoparticles. Sun and co-workers developed the synthesis of Cdots using laser ablation of a carbon target, prepared following hot-pressing a mixture of graphite powder and cement in the presence of a flow of argon gas carrying water vapour at $900{ }^{\circ} \mathrm{C}$ and $75 \mathrm{kPa} .{ }^{35} \mathrm{Li}$ et al. used a similar technique, i.e., laser passivation of carbon nanoparticles in an ordinary organic solvent for the synthesis of Cdots. ${ }^{37}$ Besides, the energy of ultrasonic waves was used to break macroscopic carbon structures such as an activated carbon $^{40}$ food waste/ethanol mixture. ${ }^{59}$ Also, chemical and electrochemical oxidations are effective top-down approaches for largescale synthesis of Cdots. Chemical oxidation of commercially available activated carbon was first introduced by Qiao et al. in order to synthesize multicolour photoluminescent Cdots. ${ }^{41}$ Cdots were prepared by electrochemical oxidation of multiwalled carbon nanotubes (MWCNTs) in the presence of an acetonitrile solution with $0.1 \mathrm{M}$ tetrabutylammonium perchlorate (TBAP) as the supporting electrolyte. ${ }^{42}$ Researchers have also used different forms of carbon sources such as graphite rods, carbon paste, carbon fibre, carbon nanotubes in order to prepare electrode materials for electrochemical generation of Cdots. ${ }^{42-44,60}$

\subsection{Synthesis of Cdots using the bottom-up approach}

As described in the preceding section, the bottom-up technique involves the synthesis of larger size Cdot particles from molecular carbon precursors. This technique allows condensation polymerization of the starting materials followed by carbonization in order to form a carbonaceous core inside the Cdot system. Presently, among all the bottom-up techniques, microwave treatment of the starting materials is the most cost- and time-effective technique for preparing high quantum yield Cdots with novel properties. Zhu et al. first introduced microwave pyrolysis of molecular precursors such as poly(ethylene glycol) (PEG-200) and saccharide (such as glucose, fructose) to synthesize carbon nanoparticles using $500 \mathrm{~W}$ microwave irradiation for 2-10 min. ${ }^{46}$ Later, Jaiswal et al. from our group reported the synthesis of optically stable and biocompatible Cdots from poly(ethylene glycol) (PEG) following microwave treatment. ${ }^{47}$ The microwave technique was used to prepare white light emitting Cdots at the single particle level (Fig. 2). ${ }^{48}$ Hydrothermal treatment of the carbonaceous precursors is another facile route for preparing Cdots. In this process, solutions of organic precursors are sealed inside hydrothermal chambers and allowed to react at high temperature and pressure. ${ }^{49-52}$

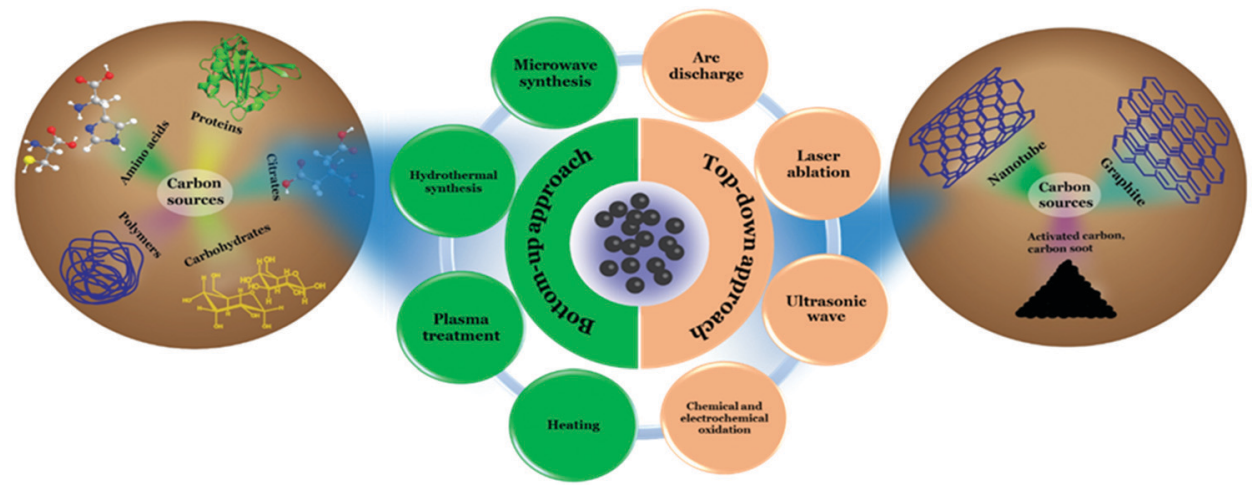

Fig. 1 Schematic illustration corresponding to different synthetic approaches used for Cdot preparation. 


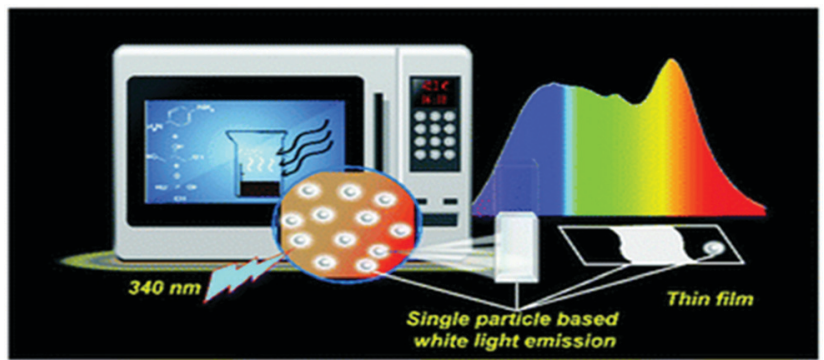

Fig. 2 Schematic representation illustrating microwave assisted synthesis of white light emitting Cdots at the single particle level. Reprinted with permission from ref. 48. Copyright 2018, Royal Society of Chemistry.

Additionally, an induction coil heating method was introduced for facile synthesis of high quantum yield Cdots. ${ }^{16}$ Heating of the aqueous solution of citric acid and ethylene diamine at $100{ }^{\circ} \mathrm{C}$ for $12-15$ min led to the formation of Cdots with $73.5 \%$ quantum yield. ${ }^{16}$ Carbonaceous nanomaterials could also be isolated from food caramels produced following direct heating. ${ }^{54}$ Also, researchers have used atmospheric plasma treatment of carbonaceous sources in order to prepare Cdots and for surface modifications following synthesis. ${ }^{56-58}$

Besides, benefits and drawbacks for all the synthesis procedures applied for Cdot preparation are represented in Table 1.

\subsection{Synthesis of crystalline Cdots}

Although initial reports indicated amorphous characteristics of Cdots, efficient modifications in the synthesis techniques, along with appropriate starting materials, have led to the generation of sufficiently graphitic crystalline nature inside carbon nanostructures. Table 2 includes mostly used synthesis techniques along with suitable starting materials to generate graphitic facets inside carbon nanostructures. Among them, microwave and hydrothermal treatment, heating, oxidation, and plasma treatment are popular techniques. For example, one-step microwave irradiation of a mixture of glycerol, $p$-phenylenediamine and $o$-phosphoric acid for $4 \mathrm{~min}$ at $450 \mathrm{~W}$ resulted in a graphitic core inside the carbon nanodot system. A distinct lattice spacing of $0.23 \mathrm{~nm}$ due to the (100) graphitic facet was identified. ${ }^{48} \mathrm{~A}$ lattice distance of $0.34 \mathrm{~nm}$ was identified during high-resolution TEM analysis for the Cdots synthesized from ethylenediaminetetraacetic acid (EDTA), 2-amino-2hydroxymethyl-propane-1,3-diol (TRIS), glycine and cadaverine by using the hydrothermal technique $\left(300{ }^{\circ} \mathrm{C}\right.$ for $\left.2 \mathrm{~h}\right) .{ }^{52}$ Powder XRD confirmed broad peaks at $18.2^{\circ}$ and $23.8^{\circ}$. Here, the second peak was attributed to the (002) plane of graphite. ${ }^{52}$ The existence of similar graphitic characteristics was confirmed during the electrochemical synthesis of Cdots from graphite rods. ${ }^{43}$ Egg-derived fluorescent Cdots showed well-resolved lattice fringes corresponding to the (100) facet of graphite (obtained using plasma treatment). ${ }^{56}$ Apart from single-crystalline graphitic Cdots, recent reports on the synthesis of polycrystalline Cdots are worth mentioning. The microwave assisted carbonization technique is found to be efficacious in generating highly crystalline domains in carbon nanostructures. Sun et al. introduced microwave radiation to aniline, ethylene diamine, and phosphoric acid to generate such polycrystalline domains in Cdots. Microwave assisted high temperature carbonization of a mixture of citric acid monohydrate and thiourea with the help of orthophosphoric acid generated Cdots with highly crystalline order. Furthermore, the degree of their crystallinity was improved by using a metal ion assisted assembly method. Addition of zinc acetate dihydrate significantly enhanced Cdot crystallinity. Detailed characteristics of such Cdot systems along with their structural insights are given in Section 4.3.2.

Table 1 Comparative representation corresponding to benefits and drawbacks for all the synthesis procedures applied for Cdot preparation

\begin{tabular}{|c|c|c|}
\hline \multicolumn{2}{|l|}{ Methods } & \multirow{2}{*}{$\begin{array}{l}\text { Carbon sources used } \\
\text { Graphite },{ }^{35,38,39} \text { biomass },{ }^{61} \text { carbon powder. }{ }^{62}\end{array}$} \\
\hline \multirow{4}{*}{$\begin{array}{l}\text { Top- } \\
\text { down } \\
\text { approach }\end{array}$} & Laser ablation & \\
\hline & Ultrasonic wave & Activated carbon. ${ }^{40}$ \\
\hline & $\begin{array}{l}\text { Chemical oxida- } \\
\text { tion techniques }\end{array}$ & $\begin{array}{l}\text { Coal activated carbon, }{ }^{41} \text { wood activated } \\
\text { carbon, }{ }^{41} \text { carbon black, }{ }^{63} \text { hair fibers, }{ }^{64} \\
\text { carbon soot. }\end{array}$ \\
\hline & $\begin{array}{l}\text { Electrochemical } \\
\text { oxidation } \\
\text { techniques }\end{array}$ & $\begin{array}{l}\text { Carbon nanotubes (CNTs), }{ }^{42} \text { graphite, }{ }^{43} \\
\text { carbon paste, }{ }^{44} \text { ethanol (EtOH). }{ }^{45}\end{array}$ \\
\hline \multirow[t]{4}{*}{$\begin{array}{l}\text { Bottom- } \\
\text { up } \\
\text { approach }\end{array}$} & $\begin{array}{l}\text { Microwave } \\
\text { treatment }\end{array}$ & $\begin{array}{l}\text { Carbohydrates, }{ }^{46} \text { polymers, }{ }^{47} \text { amino acids, }{ }^{66} \\
\text { protein molecules, }{ }^{67} \text { citric acid, }{ }^{14} \text { food grade } \\
\text { substances. }{ }^{68}\end{array}$ \\
\hline & $\begin{array}{l}\text { Hydrothermal } \\
\text { synthesis }\end{array}$ & $\begin{array}{l}\text { Carbohydrates, }{ }^{69} \text { polymers, }{ }^{70} \text { amino acids, }{ }^{71} \\
\text { protein molecules }{ }^{72} \text { citric acid, }{ }^{49} \text { biomass, }{ }^{73} \\
\text { food grade substances, }{ }^{74} \text { waste peels. }{ }^{75}\end{array}$ \\
\hline & Heating & $\begin{array}{l}\text { Citric acid, }{ }^{16} \text { food caramels, }{ }^{54} \text { glycerol, }{ }^{76} \\
{\text { polymers },{ }^{77} \text { sucrose. }}^{55}\end{array}$ \\
\hline & $\begin{array}{l}\text { Plasma } \\
\text { treatment }\end{array}$ & Egg cells, ${ }^{56}$ benzene, ${ }^{57}$ PEG. ${ }^{58}$ \\
\hline
\end{tabular}

Benefits

Fast synthesis, tunable surface states.

Easy-to-synthesize.

Large-scale synthesis, no elaborate equipment.

Size controllable, one-step synthesis, easy post-treatments.

Rapid synthesis, easy-to-synthesize, cost effective, controlled growth, eco-friendly, high quantum yield, excellent graphitic/ non-graphitic crystallinity.

High quantum yield, non-toxicity

High quantum yield, low cost, green synthesis procedure.

Rapid synthesis technique, no additives required for surface functionalization.

\section{Drawbacks}

Low quantum yield, less cost-effective and tedious size separation. High synthesis cost. Low uniformity in size distribution.

Low quantum yield, limited precursors.

Low control over size during the large-scale synthesis.

Time consuming, low product yield.

Low control over size.

Low efficiency, unpredictability over surface functionalization. 
Table 2 Synthesis procedure and corresponding observed structural characteristics of the as-prepared Cdot systems having different crystalline characteristics

\begin{tabular}{lll}
\hline Cdot systems & Synthesis procedure/carbon sources & Characteristics \\
\hline Amorphous & $\begin{array}{l}\text { (1) Laser ablation (graphite, carbon powder are the typically used } \\
\text { Cdots }\end{array}$ & $\begin{array}{l}\text { (1) No clear lattice fringes in high-resolution TEM } \\
\text { analysis. }\end{array}$ \\
& $\begin{array}{l}\text { (2) Ultrasonic wave (dextrose, activated carbon). } \\
\text { (3) Plasma treatment (PEG, benzene). }\end{array}$ & $\begin{array}{l}\text { (3) No sharp per spacing in SAED analysis (clean SAED). } \\
\text { the powder XRD pattern. }\end{array}$ \\
& (4) Chemical oxidation (candle soot, activated carbon, sucrose). & (4) Broad D and G bands $\sim 1350$ and 1560 cm ${ }^{-1}$ in Raman \\
& & spectroscopy. High $I_{\mathrm{D}} / I_{\mathrm{G}}$ ratios $(>1)$.
\end{tabular}

(5) Hydrothermal treatment (polysaccharides, proteins, citrates, biomass, food ingredients, amino acids, surfactants).

(6) Microwave treatment (biomass, citric acid, primary amine, saccharides, amino acid).

(7) Heating (food caramels, carbohydrates, long-chain amines, polymers).

Single crystalline (1) Chemical and electrochemical oxidation (carbohydrates, graphitic Cdots MWCNTs, graphite rods are the typically used carbon sources).

(1) Lattice fringes corresponding to the $d$ spacing of 0.24 , $0.28,0.32$ or $0.34 \mathrm{~nm}(1120,020,002,112$ graphitic planes respectively) are observed in high-resolution TEM analysis.

(2) Plasma treatment (egg cells).

(2) Bright spots in SAED analysis. Patterns can be either hexagonal or circular.

(3) Hydrothermal treatment (citric acid, amino acids, carbohydrates, (3) Mostly broad peak due to limited graphitization. proteins, folic acid, polymers, biomass and food grade substances). Sometimes sharp peak can be observed $\sim 2 \theta=24^{\circ}$ corresponding to the 002 plane in the powder XRD pattern.

(4) Microwave treatment (citric acid, carbohydrates, polymers, amino (4) Relatively sharp D and G bands $\sim 1350$ and $1560 \mathrm{~cm}^{-1}$ acids, proteins, biomass). in Raman spectroscopy. Broad, less intense 2D band $\sim 2700-2800 \mathrm{~cm}^{-1}$. Relatively lower $I_{\mathrm{D}} / I_{\mathrm{G}}$ ratios (mostly $\leq 1)$.

(5) Heating (citric acid, carbohydrates).

(5) Height profiles between 0.4 and $2 \mathrm{~nm}$ are observed in AFM analyses corresponding to multiple graphene layers.

Polycrystalline (1) Hydrothermal treatment (citric acid).

non-graphitic

Cdots
(2) Microwave treatment (citric acid, meta phenylene diamine-ethylene diamine mixture).

(3) Heating (glucose and ethylenediamine mixture).
(1) Coexistence of multiple well-resolved lattice fringes can be identified in high-resolution TEM analysis. (2) Bright spots are observed during SAED analysis. Mostly circular patterns corresponding to polycrystalline arrangements.

(3) Multiple sharp peaks corresponding to crystalline planes could be observed over the complete diffraction pattern.

(4) Intense and sharp G band; relatively less intense D band in Raman spectroscopy. Lower $I_{\mathrm{D}} / I_{\mathrm{G}}$ ratios $(<1)$.

(5) Thermally more stable. Degradation rate is less as compared to amorphous or partially graphitic Cdots during TGA analysis.

\section{Structures}

\subsection{Chemical structure of Cdots}

Cdots are 0D nanomaterials having size of less than $10 \mathrm{~nm}$. Typically, Cdots contain an amorphous carbonaceous core. ${ }^{78-80}$ However, depending upon starting materials and the applied reaction conditions $\mathrm{sp}^{2}$ crystalline domains may be generated, resulting in partial graphitic crystallinity in the carbon nanostructure. ${ }^{81-83}$ So far, researchers have tried to demonstrate various structural models corresponding to the carbon core such as an amorphous carbonaceous structure, ${ }^{80}$ graphitic structure, ${ }^{83}$ and diamond like structure. ${ }^{84}$ For instance, ultrasonic treatment of dextrose in $\mathrm{HCl}$ resulted in the formation of amorphous Cdots with broad diffraction characteristics in powder X-ray diffraction (powder XRD) patterns. ${ }^{78}$ A solvothermal reaction of urea and citric acid in the presence of formamide led to the formation of nitrogen doped carbonaceous $\mathrm{sp}^{2}$ domains leading to graphitic characteristics inside the structures. The high-resolution TEM results demonstrated the presence of the (100) graphitic plane corresponding to $0.22 \mathrm{~nm} d$-spacing. ${ }^{81}$ Generation of the graphitic core is achieved by using several other techniques starting with different precursors such as hydrothermal treatment of a urea and $p$-phenylenediamine mixture, ${ }^{82}$ carbonizations of glucose by the reverse micelle technique, ${ }^{83}$ hydrothermal treatment of a citric acid and ethylene diamine mixture, ${ }^{85}$ microwave assisted modification of citric acid, ${ }^{86}$ thermal oxidation of gallic acid derivatives, ${ }^{87}$ and soft-template synthesis of oleylamine-capped Cdots. ${ }^{88}$ Besides, laser irradiation to graphite powder suspension in poly(ethylene glycol) $\left(\mathrm{PEG}_{200 \mathrm{~N}}\right)$ resulted in the formation of a diamond like structure inside the carbonaceous core. ${ }^{84}$ Interestingly, following the formation of the carbonaceous core, Cdots were functionalized with various surface functional groups such as the hydroxyl, carboxyl, amide, epoxy, and amine 
controlling solubility, optical properties, and chemical reactivity of the system. ${ }^{78-88}$

\subsection{Electronic structure of Cdots}

Research groups have tried to demonstrate the electronic structure of Cdots based on the molecular orbital theory (MO theory). ${ }^{89,90}$ It has been observed that the photophysical behaviour of Cdots is highly dependent on the energy gap between the highest occupied molecular orbital (HOMO) and the lowest unoccupied molecular orbital (LUMO). Based on the dimension of the as-prepared particle, the HOMO-LUMO energy gap changes resulting in changes in emission characteristics. Further incorporation of heteroatoms (such as nitrogen, oxygen, sulphur, boron and phosphorus) ${ }^{91-93}$ or various surface functional groups (such as amines, carbonyls, amides, thiols and hydroxyls) ${ }^{89}$ in the carbon nanostructure introduces additional trap states between the HOMO and LUMO, affecting excitonic transitions and hence the photophysical properties of Cdots.

Thus, the unique chemical and electronic structure of Cdots, corresponding to size, surface functional groups and heteroatom doping, led to the generation of novel fundamental properties such as unique optical properties, good biocompatibility, excellent crystallinity and electrical conductivity, which are discussed in the following sections.

\section{Properties}

\subsection{Optical properties}

4.1.1 Absorption characteristics. Typically, Cdots exhibit strong absorbance in the UV-vis region between 240-350 $\mathrm{nm}$. The peak appearing around the $240-280 \mathrm{~nm}$ region is generally assigned to a $\pi-\pi^{*}$ transition due to the presence of the aromatic $\mathrm{C}=\mathrm{C}$ framework. Additionally, a broad peak is observed around $350 \mathrm{~nm}$ corresponding to $\mathrm{n}-\pi^{*}$ transitions due to the presence of various surface functional groups. ${ }^{82,94}$ Apart from these fundamental transition bands, additional absorption bands could be observed in some Cdot systems in the longer wavelength region due to the presence of multiple surface functional groups resulting in smaller electronic band gaps. ${ }^{14,95}$

4.1.2 Emission characteristics. Cdots are considered as an emerging class of "nanolights" because of their fascinating optical properties, high quantum yield, wavelength tunable emission and good photostability. Therefore, studying the photoluminescence (PL) properties of Cdots and corresponding utilization for multiple applications has now become a fascinating area of research.

High quantum yield. Excellent PL properties are characterized by a high quantum yield (QY), which is dependent on the synthesis procedure and starting materials. For example, 99\% QY for a Cdot system has been reported, where the Cdots were synthesized following microwave treatment of citric acid monohydrate and tris(hydroxymethyl)aminomethane. ${ }^{96}$ Hydrothermal treatment of citric acid monohydrate and $\mathrm{KH}-792$ (a silane coupling agent) results in the formation of nitrogen and silicon co-doped fluorescent Cdots with $97.32 \%$ of PL QY ${ }^{97}$ Liu et al. used folic acid to prepare nitrogen doped Cdots with a maximum QY of $94.5 \% .{ }^{50}$ Also, hydrothermal treatment of citric acid monohydrate and ethylene diamine (QY ca. 80\%), ${ }^{79}$ solvothermal treatment of citric acid and diaminonaphthalene (QY 75\%), ${ }^{98}$ induction coil heating of citric acid monohydrate and ethylene diamine (QY 73.5\%), ${ }^{16}$ microwave treatment of histidine in the presence of orthophosphoric acid (QY $44.9 \%),{ }^{66}$ and hydrothermal treatment of chitosan (QY $\left.43 \%\right)^{70}$ are prominent methods of preparing exceptionally high QY Cdots.

Excitation dependent tunable emission. Excitation dependent tunable nature in PL is one of the most important characteristics of Cdots which makes them distinct from other carbon allotropes. For example, hydrothermal treatment of urea and $p$-phenylenediamine resulted in the formation of high QY Cdots, separation of which through column chromatography yielded multiple colour emitting Cdots. Indeed, the PL mechanism was directly related to the surface states. ${ }^{82}$ Bao et al. showed that emission characteristics of Cdots can be red shifted by modifying the reaction conditions. ${ }^{99}$ Interestingly, the tunable emission characteristic is not only dependent on the applied reaction conditions but also on the precursors used for synthesis. Hu et al. proposed that Cdot emission wavelength could be tuned over the entire visible region simply by varying the reagents. ${ }^{100}$ Similarly, RGB Cdots were prepared by Lin's group using hydrothermal treatment of $p$-phenylenediamine, $o$-phenylenediamine and $m$-phenylenediamine. ${ }^{11}$ Apart from the aforementioned crucial references, a great number of reports have been published evidencing tunable emission as the foremost specification for defining Cdots. Also, PL up-conversion, ${ }^{101,102}$ chemiluminescence, ${ }^{103,104}$ and electrochemiluminescence ${ }^{105}$ are commonly observed optical properties of Cdots.

Photoluminescence (PL) mechanism of Cdots. Despite having important optical properties, which have been studied rigorously over the past decade for multiple applications, the underlying mechanisms remain to be explored because of structural heterogeneity and diverging properties depending upon reaction techniques, starting materials and applied reaction conditions. However, in the recent past, great advancement has been made in understanding the photophysical properties of Cdots, though more research is urgently needed to pinpoint the exact origin of PL properties (precisely, the excitation dependent tunable property) and corresponding mechanism. Literature reports suggest two popular models corresponding to Cdot emission. One is based on the size of particles and the other one is related to the defect states. ${ }^{43,79,99,100,106}$ For example, a few reports suggest that emission characteristics in Cdot systems typically originate from graphitic carbon cores. ${ }^{107}$ In those cases, the quantum size effect is found to be the major factor for tunable emission; also, the emission characteristics in those cases are termed intrinsic emission originating from a perfect graphitic core. Density functional theory (DFT) calculations suggest that the energy gap between the HOMO and LUMO for a single benzene ring is $\sim 7 \mathrm{eV}$, which reduces to $\sim 2 \mathrm{eV}$ for a conjugation of 20 aromatic rings. ${ }^{106}$ Similarly, Sk et al. used 
DFT and time-dependent DFT calculations to conclude that the quantum confinement effect in graphitic $\mathrm{sp}^{2}$ domains can result in tunable emission through the entire visible region. Precisely, as the size increases, the emission maximum shifts towards longer wavelength. ${ }^{107}$ The result suggests that higher the extent of graphitization inside the carbon core the lower will be the energy gap, resulting in the red-shift of the emission maximum. However, most of the reports suggest that emission characteristics in Cdot systems are controlled by multiple trap states caused by heteroatom doping or surface functional groups attached to the carbon core. The corresponding emission mechanism is known as extrinsic emission. It has been found that surface defects are mainly generated due to surface oxidation, which serves as an excitonic capture centre, giving rise to defect state emission. ${ }^{108,109}$ Increasing degree of surface oxidation introduces more trap states inside the structure resulting in the red-shift in Cdot emission. Apart from oxygen containing surface functional groups, nitrogen doping (resulting in $-\mathrm{NH}_{2},-\mathrm{CONH}_{2},-\mathrm{CHNR}$ surface functional groups) and doping with sulphur, boron, and phosphorus result in significant changes in PL QY along with tuning of the emission wavelength. ${ }^{14,47,100}$ Fig. 3 illustrates the corresponding surface state controlled extrinsic emission in Cdots.

Although the aforementioned reports demonstrate carbonization of the precursors during the course of the reaction resulting in photoluminescence (PL), several other reports published in recent times indicate formation of highly fluorescent molecular fluorophores during the bottom-up synthesis of Cdots. ${ }^{110-113}$

For example, formation of such fluorophores is identified when citric acid and aromatic amine are used as the precursors. ${ }^{110-113}$ So generated fluorophores can be present separately with the carbogenic core or can be attached to the carbogenic core generating multiple emitting sites. In such cases, the emission characteristics are neither solely controlled by the Cdot core (resulting in intrinsic emission) nor by the surface functional groups (resulting in extrinsic emission). Rather, tunable broad emission could be observed because of the ensemble of multiple emission centres (resulting in superposition of emissions) caused by carbogenic cores along with organic fluorophores and their aggregates. In 2011, Giannelis and co-workers pursued temperature dependent studies in order to understand the formation mechanism of carbogenic particles from citric acid and ethanolamine. ${ }^{110}$ In the work, they proposed that PL properties in Cdots are determined by both the carbogenic core and molecular fluorophores. In the Cdots, obtained by low temperature pyrolysis, molecular fluorophores dominate the emission characteristics, whereas during high temperature pyrolysis the carbogenic core starts forming,
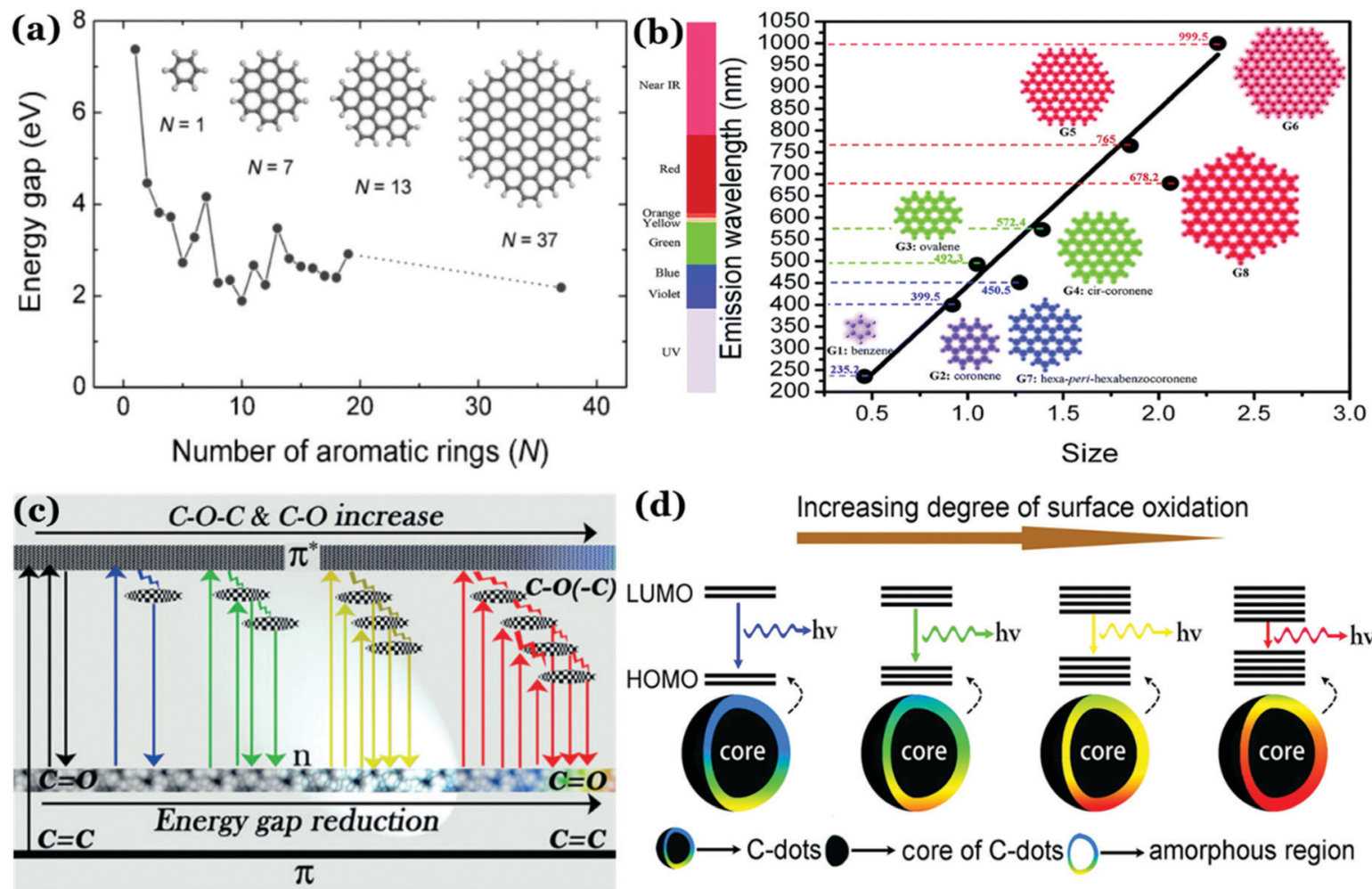

(d)

Increasing degree of surface oxidation

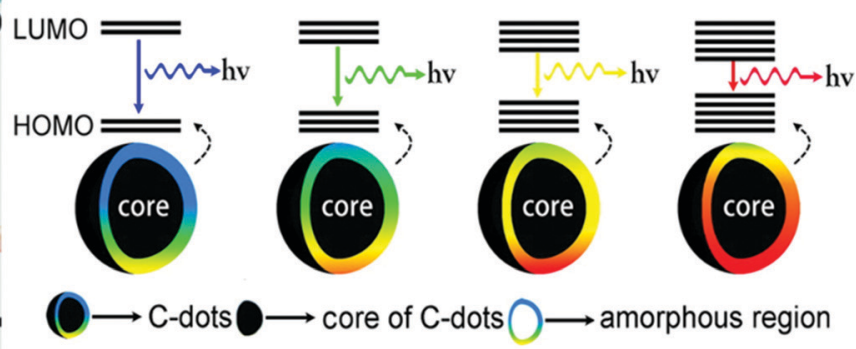

Fig. 3 (a) Variation in the energy gap corresponding to $\pi-\pi^{*}$ transitions as a function of the number of aromatic rings, calculated using a DFT study. Reprinted with permission from ref. 106. Copyright 2010 John Wiley \& Sons. (b) Change in emission wavelength as a function of diameter, calculated using the TDDFT method. Reprinted with permission from ref. 107. Copyright 2014 Royal Society of Chemistry. (c) Representation of the tunable emission in Cdots controlled by different oxygen containing surface functional groups. Reprinted with permission from ref. 100. Copyright John Wiley and Sons 2015. (d) Proposed model for the surface group controlled tunable emission in Cdots. Reprinted with permission from ref. 82 . Copyright 2016 American Chemical Society. 


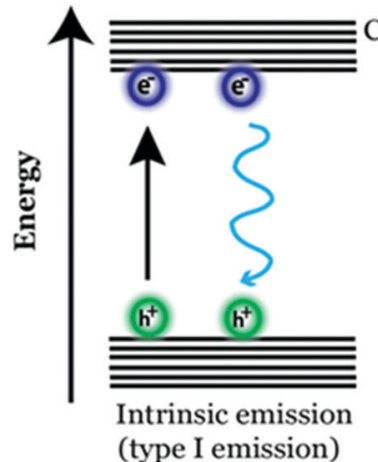

(type I emission)

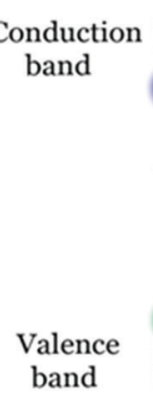

Intrinsic emission extrinsic emission (due to surface defects) (type II emission)

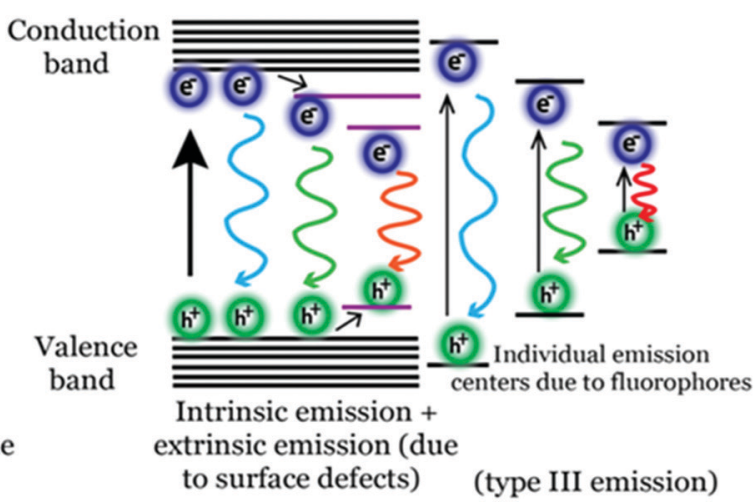

Fig. 4 Schematic representation corresponding to the emission mechanism in Cdot systems.

which dominates the emission characteristics. Later in 2015 , Song et al. showed the formation of a molecular fluorophore termed IPCA (imidazo[1,2- $a$ ]pyridine-7-carboxylic acid) along with its derivatives during the hydrothermal reaction between citric acid and ethylene diamine. ${ }^{111}$

The exact fluorophore structure was confirmed by mass spectra, NMR spectra and elemental analysis following successful separation of the product by column chromatography. Also, molecular fluorophores have been identified during Cdot synthesis using aromatic amines. Zhang et al. synthesized red emitting Cdots from $p$-phenylenediamine using the solvothermal method. ${ }^{112}$ Based on spectroscopic studies, they proposed the generation of different molecular states due to the formation of nitrogen containing organic fluorophores and thus the origin of the longer wavelength emission band in Cdot systems. Fig. 4 illustrates three possible mechanisms corresponding to tunable emission characteristics in Cdots.

It is important to note that the formation of such organic molecular fluorophores and their effect over Cdot emission are observed for relatively lower temperature $\left(<200{ }^{\circ} \mathrm{C}\right)$ synthesis processes. Thus, we believe that incomplete carbonization of the starting materials would result in the formation of such fluorescent organic molecular species with carbogenic cores resulting in relatively complicated type 3 emission. Now, whether those fluorophores can be completely separated from carbogenic cores or what happens to the emission characteristics of Cdots after complete separation needs to be addressed.

4.1.3 Charge transfer properties. Apart from being a good optical material, Cdots are conducive to efficient charge transfer. They can act as both electron donors and acceptors depending upon the surface functional moieties. Wang et al. first reported that photoexcited Cdots can be quenched by both electron donors (such as the $N, N$-diethylaniline) and electron acceptors (such as 4-nitrotoluene and 2,4-dinitrotoluene) suggesting Cdots as excellent electron acceptors and electron donors. ${ }^{114}$ Indeed, findings of such photoinduced redox characteristics in Cdots created new opportunities for their potential applications in efficient light-to-energy conversion. Zhang et al. introduced Cdots in silver orthophosphate $\left(\mathrm{Ag}_{3} \mathrm{PO}_{4}\right)$ to enhance photocatalytic activity (Fig. 5). ${ }^{115}$ Results show that excellent PL up-conversion and charge storage abilities of Cdots synergistically enhance the photocatalytic activity of the complex. A rapid charge transfer phenomenon was also observed from Cdots to graphene oxide using steady state and ultrafast spectroscopy, which resulted in strong PL quenching in Cdots. ${ }^{116}$ It is important mentioning here that, although the charge transfer phenomenon in Cdots has been extensively reported in the recent past, direct evidence corresponding to efficient photoinduced charge separation is yet to be reported and established. In addition, structural inhomogeneity, i.e., lack of proper order inside the Cdot structure results in strong electron-phonon scattering, thus hindering efficient charge transfer through $\pi$-stacks. Therefore, it is believed that generation of large crystalline domains in the Cdot nanostructure can facilitate efficient charge separation and delocalization, and thus improve device performances.

\subsection{Electrical conductivity}

Electrical conductivity in Cdot systems is another important property, which has been investigated in recent times. Zhang et al. reported the conducting nature of Cdots, synthesized from electrochemical ablation of graphite. ${ }^{117}$ Later, poly(4styrene sulphonate)-stabilized Cdots (PSS-Cdots) were synthesized, which showed excellent conducting nature. ${ }^{118}$ The conducting nature was characterized through current-voltage $(I-V)$ characteristic studies using conductive atomic force microscopy (C-AFM). The $I-V$ plots for the PSS-Cdot system

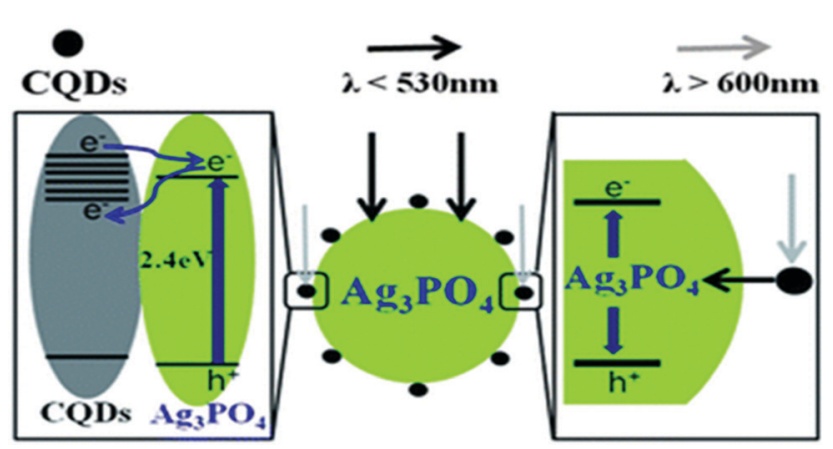

Fig. 5 Schematic representation corresponding to Cdot assisted improved photocatalytic activity of a $\mathrm{Cdot} / \mathrm{Ag}_{3} \mathrm{PO}_{4}$ complex. Reprinted with permission from ref. 115. Copyright 2012 Royal Society of Chemistry. 
(Fig. 6a) showed a sharp change between $-0.1 \mathrm{~V}$ to $0.1 \mathrm{~V}$ followed by saturation in the measured current, demonstrating the as-synthesized PSS-Cdot as a natural conductor. Further, the cyclic voltammetry (CV) technique was used to probe such conducting properties in the Cdot system (Fig. 6b). Bhattacharjee et al. proposed that surface modification with PSS generated several defect states on the Cdot surface, which allowed facile electron transfer regardless of the work function, resulting in symmetric $I-V$ characteristics in both the directions. They surmised that application of high electric fields during the measurements allowed removal of the electrons from the lower energy level of PSS-Cdots, sandwiched between gold and ITO work functions (Fig. 6c) resulting in conductivity along with exhibition of metallic characteristics.

Recently, our group has synthesized nitrogen doped Cdots from citric acid monohydrate and ethylene diamine using the induction coil heating method. ${ }^{119}$ Interestingly, apart from being good optical materials, they were found to be electrically conducting in nature. $I-V$ characteristics of the Cdot film, demonstrating conducting nature, was attributed to the presence of $\mathrm{sp}^{2} \mathrm{C}-\mathrm{C}$ domains inside the carbon nanostructure, allowing conjugation of the adjacent $\pi$-bonds to form the $\pi$-and $\pi^{*}$-bands. However, when such conducting Cdots were doped inside a polymer (polypyrrole; PPy in this work), the conductivity of the resultant composite (termed as Cdot-PPy) was found to be increased significantly (Fig. 7a-d). According to the study, the PPy film showed a conductivity of $0.23 \mathrm{mS} \mathrm{m}^{-1}$ whereas the same for the Cdot-PPy film was measured to be $2.60 \mathrm{mS} \mathrm{m}^{-1}$
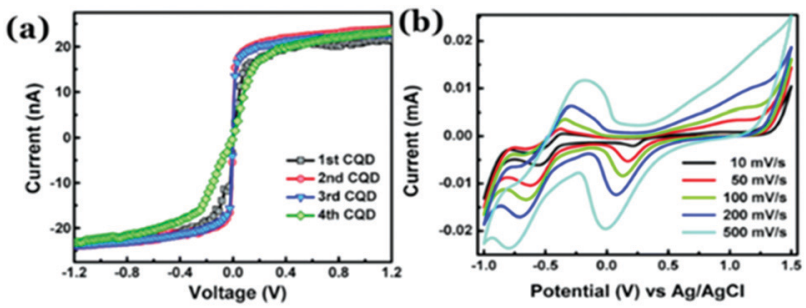

(c) trap

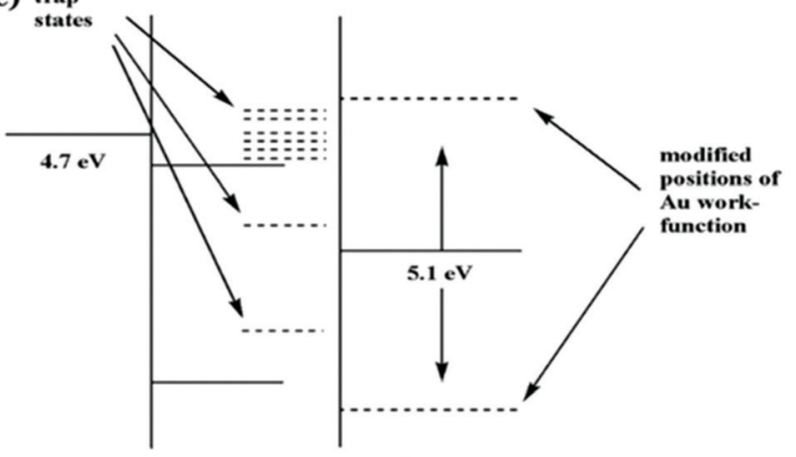

ITO

CQD

Au

Fig. 6 (a) $I-V$ characteristic plots of PSS-Cdots recorded from different spots. (b) Cyclic voltammetry (CV) plot for aqueous PSS-Cdot dispersion at different scan rates. (c) Schematic representation corresponding to the band diagram of PSS-Cdot sandwiched between an indium tin oxide coated glass substrate (ITO) and a gold coated AFM tip (Au). Reprinted with permission from ref. 118. Copyright 2015 Royal Society of Chemistry.
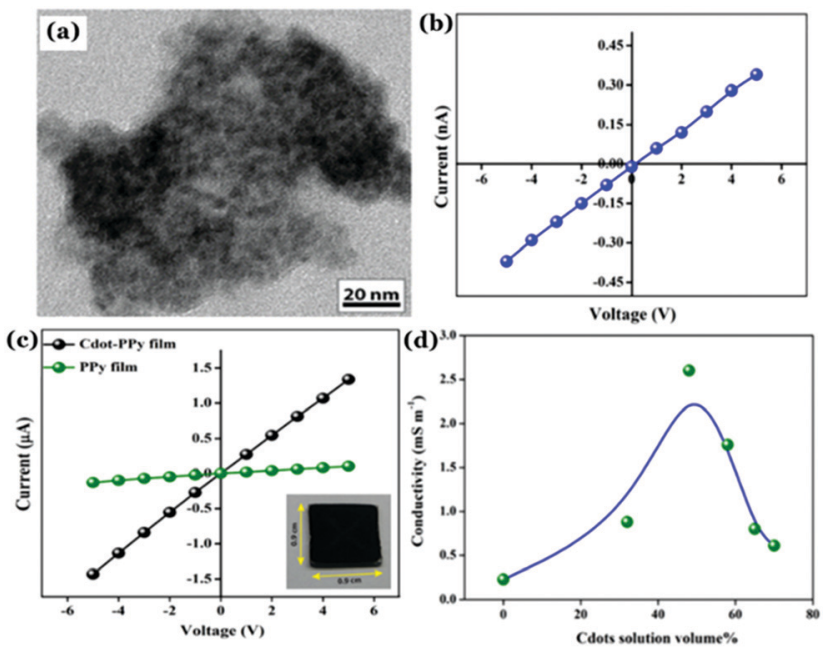

Fig. 7 (a) TEM image of the Cdot-PPy nanocomposite material. Plots of $I-V$ characteristics corresponding to (b) Cdot film and (b) Cdot-PPy and PPy films. (d) Change in the conductivity of the composite due to the variation of Cdot content in the composite film. Reprinted with permission from ref. 119. Copyright 2016 American Chemical Society.

under identical measurement conditions. Concentration dependent changes in conductivity values of the composite have also been studied and are shown in Fig. 7d. As represented in Fig. 7d, the conductivity of the composite film increased significantly with increasing Cdot loading percentage. Improved conductivity in the polymer following Cdot doping was attributed to the efficient electron withdrawing tendency by Cdots.

PPy is a p-type semiconductor where holes are the prime charge carriers. It was surmised that doping with Cdots helped increase the positive charge density in the polymeric backbone resulting in the enhancement of the composite conductivity. It is worth mentioning that the discovery of conducting nature in Cdot systems is remarkable, substantiating their potentials for being used in green flexible nanoelectronics. However, a poor crystalline arrangement inside the Cdot structure is a limitation, which is yet to be addressed for achieving high conductivity and fabricating efficient electronic devices.

\subsection{Crystalline properties}

Unlike other allotropes of carbon, Cdots were initially considered as the amorphous allotrope. Initial reports indicate the presence of a large extent of $\mathrm{sp}^{3}$ carbon atoms inside Cdot structures. $^{79,120-123}$ They exhibit a broad diffraction pattern in the powder XRD analysis with no characteristic lattice fringes corresponding to interlayer spacings. In addition, broad D- and G-bands could be observed in Raman spectrometry along with high intensity ratios between the two, i.e., $I_{\mathrm{D}} / I_{\mathrm{G}}$ ratios. Of late, researchers have brought novel ideas of generating crystalline nature in carbon nanostructures. The crystal qualities in Cdots can be broadly classified into two categories consisting of either a graphitic crystalline core or a high degree of non-graphitic crystallinity, which are elaborated in the following sections.

4.3.1 Graphitic Cdots. Formation of graphitic crystallinity inside the Cdot structure has been observed extensively. So far, 
multiple research groups have proposed generation of $\mathrm{sp}^{2}$ bonded $\mathrm{C}-\mathrm{C}$ domains, organized into a $2 \mathrm{D}$ honeycomb lattice inside the carbon core. Precisely, most of the graphitic Cdots exhibit a moderately sharp peak around $2 \theta=24^{\circ}$, corresponding to the (002) plane. Besides, interlayer spacings of $0.24,0.28,0.32$ or $0.34 \mathrm{~nm}$ are mostly observed during highresolution TEM and inverse fast Fourier transform (IFFT) analysis, identified as the graphitic planes. In selected area electron diffraction (SAED), observation of hexagonal spots further confirms their graphitic characteristics. In Raman spectroscopy analysis, the $I_{\mathrm{D}} / I_{\mathrm{G}}$ ratios are mostly found to be lower as compared to those of completely amorphous Cdot structures. For instance, Moon et al. reported one-step hydrothermal treatment of fumaronitrile (Fig. 8a), which led to the formation of a highly ordered graphitic structure in Cdots. ${ }^{124}$ Formation of the same was confirmed via high-resolution TEM analysis (with a measured $d$ spacing of $0.243 \mathrm{~nm}$ ) (Fig. 8b), Raman spectrometry (Fig. 8c) and near-edge X-ray absorption fine structure (NEXAFS) measurements (Fig. 8d and e). Detailed NEXAFS analyses suggest the presence of the $\mathrm{C} 1 \mathrm{~s}$ core level electronic transition into $\pi^{*}$ symmetry of the graphene Brillouin zone (termed $\mathrm{CK}_{1}$ ) and $\sigma^{*}$ symmetry states (termed $\mathrm{CK}_{6}$ ). Besides, the intensity ratios of $\mathrm{CK}_{3} / \mathrm{CK}_{1}\left(\mathrm{CK}_{3}\right.$ corresponding to $\mathrm{C}-\mathrm{O}$ transition appearing at $287.2 \mathrm{eV}$ ) and $\mathrm{CK}_{6} / \mathrm{CK}_{1}$ near the $\mathrm{C}$ K-edge were found to be much lower as compared to other non-graphitic Cdots, suggesting the formation of a higher degree of $\mathrm{sp}^{2}$-hybridized carbonaceous structure (Fig. 8e).
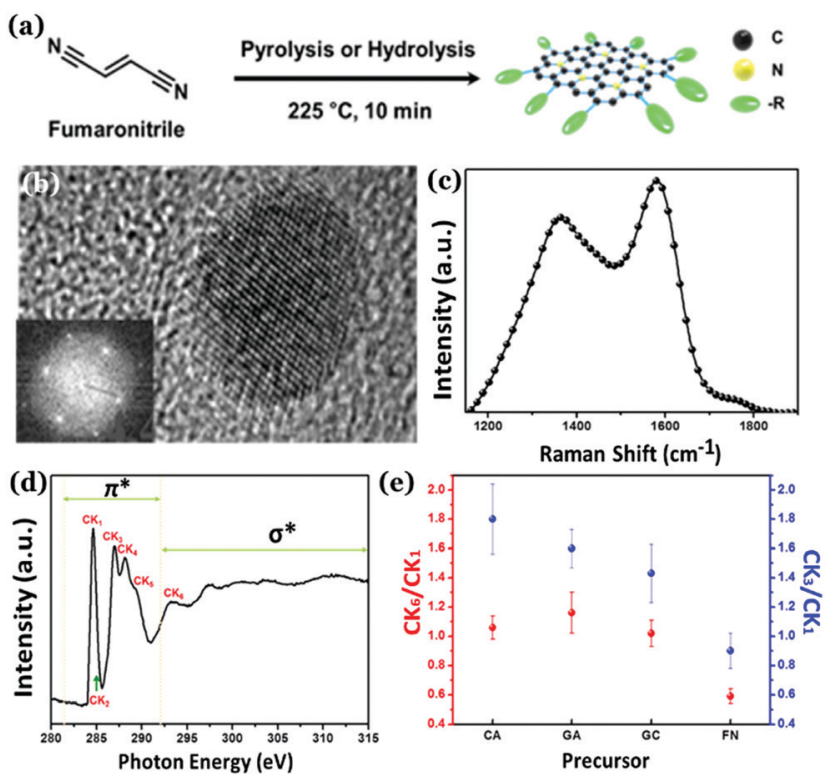

Fig. 8 (a) Synthesis of nitrogen doped highly graphitic Cdots. (b) Highresolution TEM image and the fast Fourier transform (FFT) image of the as-synthesized Cdots. (c) Raman spectra of the graphitic Cdots with a high intensity G-band. (d) $\mathrm{C}$ K-edge NEXAFS spectrum corresponding to as-synthesized graphitic Cdots. (e) The $\mathrm{CK}_{3} / \mathrm{CK}_{1}$ and $\mathrm{CK}_{6} / \mathrm{CK}_{1}$ intensity ratios for as-prepared highly graphitic Cdots and Cdots derived from other precursors. Reprinted with permission from ref. 124. Copyright 2016 American Chemical Society.
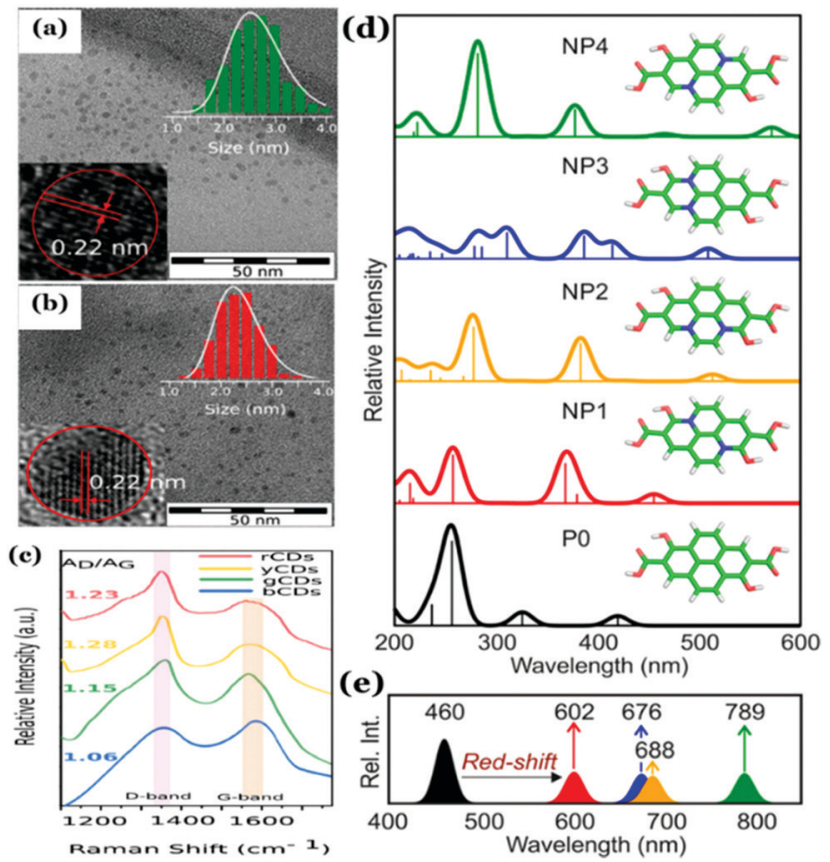

Fig. 9 ( $a$ and b) TEM images and high-resolution TEM images of assynthesized Cdots with a measured $d$-spacing of $0.22 \mathrm{~nm}$ corresponding to the (100) graphitic lattice. (c) Raman spectra with an intense G-band corresponding to Cdots collected from different fractions confirming the formation of the graphitic structure. ( $d$ and e) Proposed graphitic structure and corresponding changes in optical properties as a function of the extent of graphitization inside the carbonaceous core and the optical band gap. Reprinted with permission from ref. 81. Copyright 2017 American Chemical Society.

Also, Hola et al. proposed the formation of aromatic frameworks inside the carbonaceous core during Cdot synthesis (Fig. 9) ${ }^{81}$ Indeed, based on experimental and theoretical analyses, they proposed that such a graphitic network, generated inside the core, does have significant contribution in controlling the photophysical properties of Cdots. According to the report, with increasing nitrogen content along with the size of conjugated $\mathrm{sp}^{2}$ domains the HOMO-LUMO band gap reduces significantly resulting in the shifting of the emission maxima. Later, formation of a similar type of structure has been proposed by Shi et al. ${ }^{125}$ Though generation of such single crystalline graphitic domains results in novel optical properties such as the excitation dependent tunable emission and high QY, the extent of such domains is limited. Indeed, much research is still required to improve the graphitic crystalline quality in Cdots, which will be effective for further efficient energy and electron transfer and/or transportation.

4.3.2 Non-graphitic crystalline structures. In recent times, researchers have discovered new techniques of improving the crystal quality inside Cdot systems. Such types of Cdots consist of high degree of polycrystalline domains inside the structures, as characterized by high-resolution TEM, SAED, Raman spectroscopy, and powder XRD analyses. Unlike graphitic Cdots, the powder XRD patterns of such Cdot systems consist of several intense sharp peaks, demonstrating their polycrystalline characteristics. 

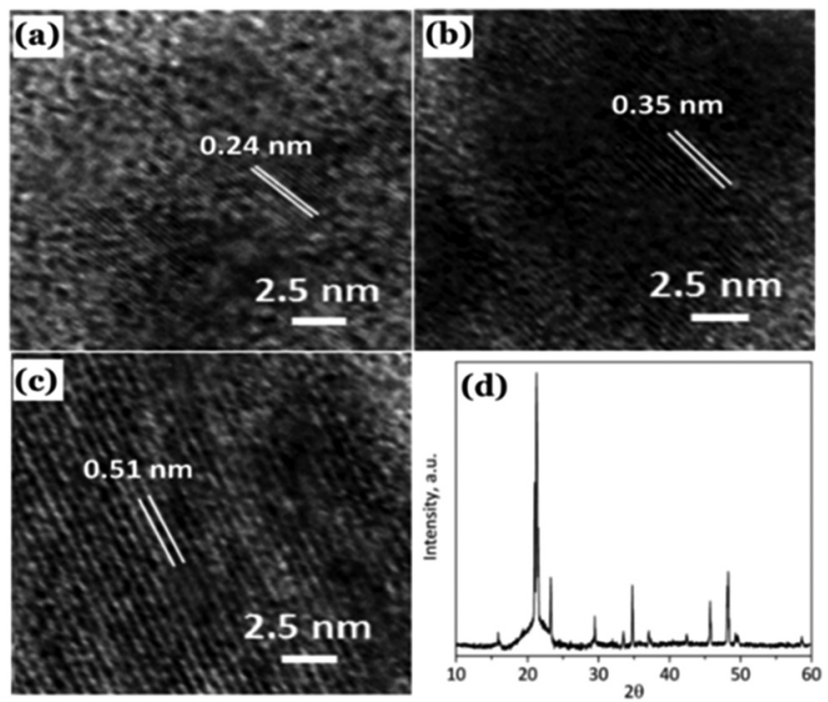

Fig. $10(a-c)$ Observed lattice planes in the as-prepared Cdots reported by Sun et al. (d) Powder XRD pattern of the Cdots. Reproduced with permission ref. 126. Copyright 2015 Royal Society of Chemistry.

Polycrystalline Cdots with bright fluorescence were synthesized by microwave treatment $(800 \mathrm{~W}, 40 \mathrm{~s})$ of aniline, ethylene diamine, and phosphoric acid by Sun et al. ${ }^{126}$ As illustrated in the report, the polycrystalline nature was characterized using high-resolution TEM and powder XRD analysis (Fig. 10) and the observed optical properties in the as-prepared Cdot system were attributed to co-doping with $\mathrm{N}$ and $\mathrm{P}$. Though a few of the lattice planes obtained during high-resolution TEM analysis matched well with the graphitic characteristics, additional non-graphitic peaks could be identified during powder XRD pattern analysis suggesting the presence of both characteristics in the as-prepared Cdots.

Recently, our group has demonstrated that incorporation of phosphorus inside the carbon nanostructure under controlled reaction conditions can generate excellent crystalline properties. ${ }^{127}$ Microwave treatment (600 W, $5 \mathrm{~min}$ ) of citric acid monohydrate and thiourea in the presence of orthophosphoric acid led to the formation of highly crystalline Cdots (Fig. 11a-c). Results confirmed amorphous characteristics when there was no phosphorus present in the Cdot structure. However, doping with phosphorus and increasing the input concentration of the same resulted in improved crystalline nature. A high degree of multicrystalline nature was observed during high-resolution TEM analysis as well. Theoretical refinements of the observed XRD pattern and other experimental evidence showed the formation of fused polycyclic fragments corresponding to citrazinic acid during the carbonization of starting materials. Multiple layers of such fragments connected via phosphate groups brought crystalline order in the Cdot system. The orthorhombic arrangement of such a phosphate ligated bi-layer structure along with unit cell parameters of $a=10.6211 \AA$, $b=5.3899 \AA, c=3.7471 \AA$ and $\alpha=\beta=$ $\gamma=90^{\circ}$ was found to be matching well with the obtained XRD pattern. In addition, the observed structural characteristics due to crystalline arrangements in the carbon nanodot systems are summarized in Table 2 .
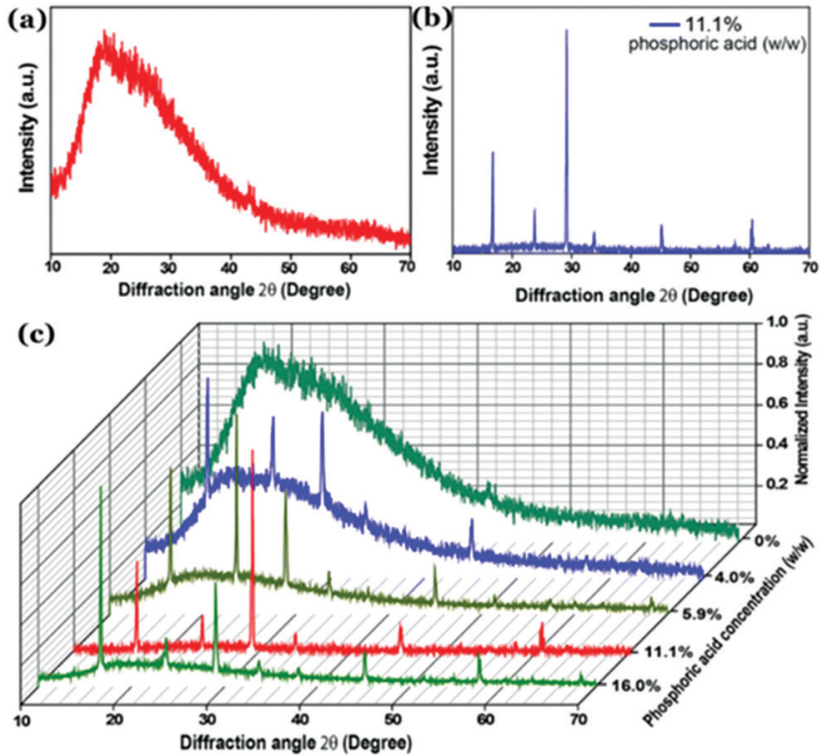

Fig. 11 Observed powder XRD pattern observed for (a) Cdots without phosphorus doping and (b) phosphorus doped Cdots (P-Cdots). (c) Powder XRD patterns of P-Cdots with different phosphoric acid input concentrations, added to the reaction mixture. Reprinted with permission ref. 127. Copyright 2018 Royal Society of Chemistry.

Self-assembly via surface complexation with metal ions is an important technique to further enhance crystallinity in Cdots. For example, $\mathrm{Zn}^{2+}$ ion induced assembly of Cdots was found to improve the extent of crystallinity in the Cdot structure. ${ }^{128}$ Cdots were synthesized from citric acid monohydrate and thiourea in the presence of orthophosphoric acid. Addition of orthophosphoric acid allowed exothermic, rapid polymerization and high temperature carbonization of the precursors. The as-prepared Cdots consisted of excess free $\mathrm{PO}_{4}{ }^{3-}$ groups over the surface, which resulted in selective binding with $\mathrm{Zn}^{2+}$ ions, thus forming highly crystalline Cdot assemblies. ${ }^{128}$ Interestingly, formation of such crystalline domains was found to assist in facile electrolyte transportation, which is detailed in the successive section.

Generation of well-ordered crystal structures in Cdot systems is important for realization of their application potential. A detailed literature study reveals that the noble characteristics in Cdots, reported so far, are based on amorphous Cdots or Cdots having limited graphitic crystallinity. Although they exhibit excellent optical properties along with desired structural flexibility, based on the synthesis procedure and starting materials, one of the biggest challenges is explaining the origin of their luminescence. Although, based on the observed phenomena, different research groups have depicted plausible luminescence mechanisms, a generalized PL mechanism still remains to be established. We believe that lack of structural insight is the other reason, which mostly restricts the establishment of a universal PL mechanism. It is also believed that generation of well-ordered crystalline domains in Cdot structures is desirable not only to have proper structural insight and in understanding the PL mechanism but also for improved 
application potentials, which are described in the following sections.

\section{Applications}

The crystalline nature of Cdots can be categorized into two classes: (1) single-crystalline graphitic Cdots and (2) polycrystalline Cdots. Recent advances on their application potentials are highlighted in the following sections.

\subsection{Application in photovoltaics}

Efficient harnessing of solar energy by using photovoltaic devices such as the solar cells is one of the most viable ways of solving world's ever-increasing energy demands. Presently, significant improvement in the power conversion efficiency (PCE) value has been achieved in all types of solar cells by means of novel materials synthesis, materials restructuring and technology optimization. ${ }^{129,130}$ However, it is still challenging to generate sufficient electricity by using these state-of-the-art solar cells in cloudy, rainy or any other kinds of low light conditions. ${ }^{129}$ Besides, the presently used photovoltaic devices are limited due to several drawbacks, inter alia, high cost, lower efficiency, and use of environmentally hazardous materials that further necessitate development of green alternatives. ${ }^{130}$ Currently, organic Cdots with graphitic crystallinity are emerging as a preferred sustainable alternative for light harvesting applications due to appropriate surface area, tunable bandgap and excellent electron accepting tendency and are being frequently used in organic dye sensitized, perovskite and silicon-based solar cells. ${ }^{32}$ Herein we discuss a few essential reports on the photovoltaic application of crystalline Cdots. So far, utilization of GQDs in fabricating solar cells has been extensive. ${ }^{131-137}$ For example, Li et al. prepared GQD-based bulk heterojunction (BHJ) polymer solar cells using an ITO/PEDOT:PSS/P3HT:GQDs/Al structure with an open-circuit voltage of $0.67 \mathrm{~V}$ with a measured PCE of $1.28 \% .^{137}$ Notably, the good electron accepting tendency of Cdots in the presence of poly(3-hexylthiophene) (P3HT) is the key to fabricate solar cells with efficiency $\sim 1$. Kim and coworkers used graphitic Cdot supported silver nanoparticles (Cdot-Ag) to improve the PCE of solar cells significantly. Clustering of silver nanoparticles on the Cdots has a substantial effect on the plasmonic phenomena and absorption of the nanoparticles, leading to increased PCE of PTB7: $\mathrm{PC}_{71} \mathrm{BM}$-based $\mathrm{BHJ}$ solar cells up to $8.31 \% .{ }^{138}$ The as-prepared Cdot-Ag nanoparticle system showed an internal quantum efficiency of $100 \%$ signifying that every absorbed photon resulted in a separated pair of charge carriers as well as all photogenerated carriers were collected at the electrodes without any further loss. ${ }^{138}$ Additionally, electrode interfacial buffer layers are crucial as they allow charge extraction and also prevent charge recombination at the interface. In this regard, Cdots can be utilized as effective charge transport materials to improve efficiencies in solar cells. ${ }^{32}$ Yan et al. prepared graphitic Cdots via the chemical vapour deposition (CVD) method, which was used as the electron transport layers to improve the thermal stability of polymer solar cells. ${ }^{139}$
Generally, zinc oxide $(\mathrm{ZnO})$ is used as an electron-transporting layer (ETL) in organic solar cells. However, due to surface defects and energy band mismatch with the photoactive layers, a lightsoaking process is usually required to achieve a high device performance. ${ }^{140}$ Ma et al. prepared graphitic N,S co-doped Cdots and used the same as a surface modifier for the $\mathrm{ZnO}$ layer. Results indicated higher PCE and removal of the light-soaking effect for ZnO:N,S-Cdot based inverted organic solar cells. The improvement was attributed to $\mathrm{ZnO}$ surface defect passivation by N,S-Cdots (Fig. 12a-c) along with their excellent photoelectric properties due to crystallinity. ${ }^{140} \mathrm{Cdot}$ modified $\mathrm{TiO}_{2}$ was used as a cathode interfacial layer in order to develop an inverted organic photovoltaic device. ${ }^{141}$ Carboxylic acid $(-\mathrm{COOH})$ groups present on the Cdot surface induced formation of a self-assembled monolayer with $\mathrm{TiO}_{2}$, which further allowed lowering the energy barrier for electronic transportation along with a reduction in the incompatibility between the metal oxide and organic active layers. ${ }^{141}$

Dye-sensitized solar cells typically consist of a photoelectrode, electrolyte, and counter electrode. Cdots can be utilized as the sensitizer or the component of the photoanode and counter electrodes. ${ }^{142}$ For example, Cdot induced poly aniline (PANI) nanocomposite films have been used as counter electrodes for dye-sensitized solar cells with $7.45 \%$ measured PCE, higher than that of Pt $(7.37 \%)$ or PANI (5.60\%) counter electrodes. ${ }^{143}$ Introduction of Cdots into PANI resulted in 3.5 times higher surface area (which was measured to be $43.6 \mathrm{~m}^{2} \mathrm{~g}^{-1}$ ) than pristine PANI $\left(12.43 \mathrm{~m}^{2} \mathrm{~g}^{-1}\right)$ and high electrical conductivity $\left(774 \mathrm{~S} \mathrm{~cm}^{-1}\right)$, leading to improved PCE. Results showed that the interior of Cdots holds a high degree of graphitic facets and integration of the same inside PANI resulted in higher linearity of the polymer chains (as confirmed from XRD results), which enhanced the overall crystallinity of the Cdot-PANI film. Integration of Cdots into transparent CoSe is an efficient way to improve the efficiency of bifacial dye-sensitized solar cells. ${ }^{144}$ Under illumination, photogenerated electrons from Cdots helped in increasing the electron density in CoSe, thereby promoting reduction of $\mathrm{I}^{3-}$ to $\mathrm{I}^{-}$. The rear efficiency was increased to $7.01 \%$ by using the as prepared Cdot-CoSe counter electrode in a dye-sensitized solar cell (Fig. 12d-f). ${ }^{144}$

On the other hand, perovskite solar cells have emerged as an important class of photovoltaic devices because of their high efficiency. ${ }^{145-149}$ Notably, introduction of hybrid organic-inorganic materials during solar cell device fabrication allowed researchers to achieve PCE $>30 \% .{ }^{150}$ Effects of Cdots in designing perovskite based solar cells are noteworthy. $\mathrm{N}$ doped graphitic Cdots were used to devise $\mathrm{CH}_{3} \mathrm{NH}_{3} \mathrm{PbI}_{3}$ perovskite film based solar cells. ${ }^{151}$ Introduction of Cdots remarkably enhanced the photocurrent and PCE. Addition of Cdots allowed effective passivation resulting in elimination of surface trap states. At the same time, their structural arrangement helped in improving charge transport through the layers. Measured PCEs for the cells were found to be $12.12 \pm 0.28 \%$ and $15.93 \pm 0.15 \%$ for a pristine device and the cell following Cdot introduction, respectively. ${ }^{151}$ In the case of heterojunction perovskite solar cells, ETLs play crucial roles in charge extraction. Using a Cdot/ $/ \mathrm{TiO}_{2} \mathrm{ETL}$ in conjugation 

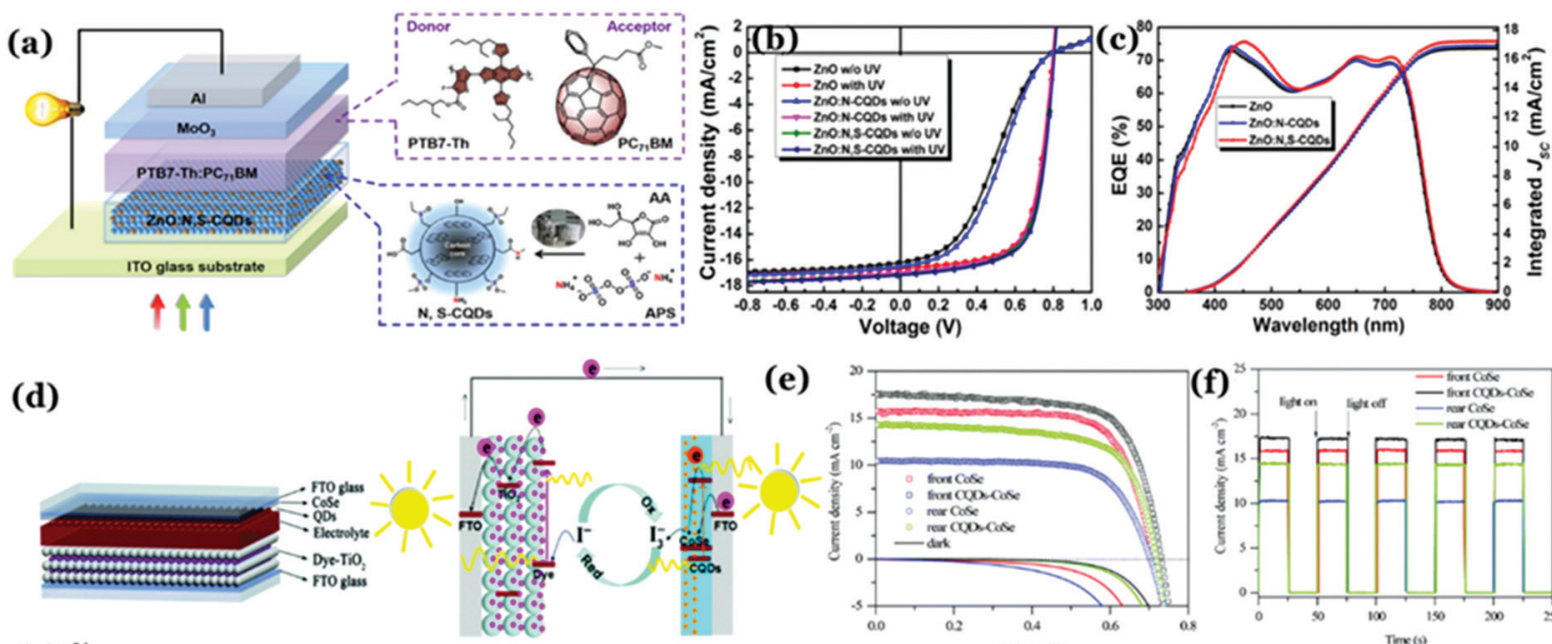

(e)
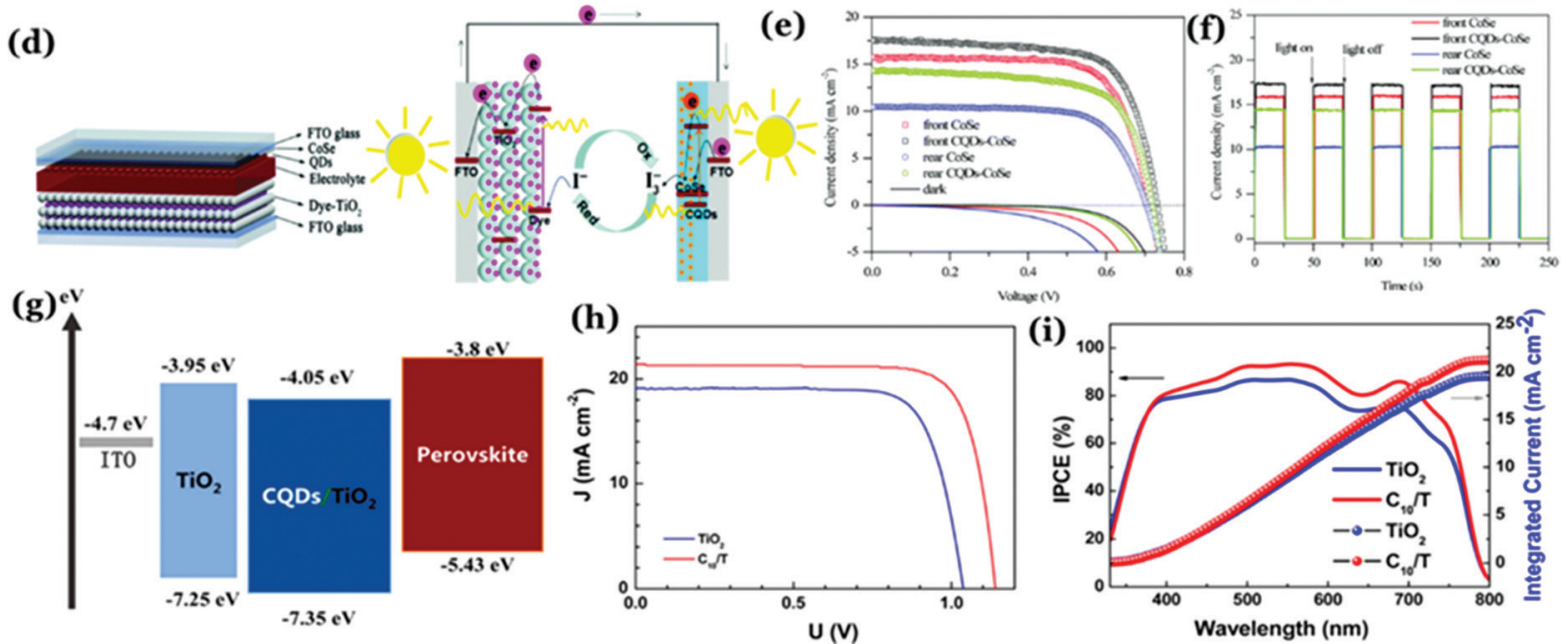

Fig. 12 (a) Schematic representation of a Cdot based organic solar cell device. (b) $J-V$ curves without and with UV treatment and (c) external quantum efficiency (EQE) spectra following UV treatment corresponding to $\mathrm{ZnO}-$, ZnO:N-Cdot-, and ZnO:N,S-Cdot based PTB7-Th:PC 71 BM solar cells. Reprinted with permission from ref. 140. Copyright 2019 American Chemical Society. (d) Charge transfer processes in Cdot based dye-sensitized solar cells. (e) $J-V$ curves and (f) on-off switching characteristics of the cells. Reprinted with permission from ref. 144. Copyright 2017 Royal Society of Chemistry. (g) Schematic representation of energy levels of a Cdot based perovskite solar cell. (h) J-V curves and (i) incident photon conversion efficiency (IPCE) curves corresponding to devices. Reprinted with permission from ref. 152. Copyright 2017 American Chemical Society.

with a planar n-i-p heterojunction 19\% PCE was achieved under standard illumination conditions. ${ }^{152}$ It was observed that the Cdot/ $\mathrm{TiO}_{2}$ composite helped increase both the open circuit voltage as well as short-circuit current density as compared to the pristine $\mathrm{TiO}_{2}$ film. Spectroscopic analyses revealed that the presence of Cdots with crystalline facets resulted in increasing electronic mobility and electron extraction ability, and appropriately matched energy levels leading to a remarkable increase in open-circuit voltages $\left(V_{\mathrm{oc}}\right)$ and short-circuit current density $\left(J_{\mathrm{sc}}\right)($ Fig. $12 \mathrm{~g}-\mathrm{i}){ }^{152}$

Crystalline silicon nanostructure based solar cells hold intense research interest as well, due to their considerably high efficiency and long lifetime. It was revealed that direct coating of single-crystalline Cdots over silicon nanowires (SiNWs) actually helps in improving efficiencies of photovoltaic cells. ${ }^{153}$ Electrical analysis corresponding to core-shell heteroconjugation revealed 9.1\% PCE along with a measured $V_{\text {oc }}$ and $J_{\mathrm{sc}}$ of $0.51 \mathrm{~V}$ and $30.09 \mathrm{~mA} \mathrm{~cm}{ }^{-2}$, respectively. High $J_{\mathrm{sc}}$ was attributed to strong light trapping of SiNW arrays and 3D core-shell geometry with increased interfacial area of the Cdot/SiNW device. Also, the device performance was highly dependent on Cdot layer thickness. Importantly, the applied Cdot layer not only acted as the hole transport medium but also functioned as the electron blocking layer in the device, thus reducing electronic recombination. ${ }^{153}$
Thus, previous reports suggest that Cdots with singlecrystalline graphitic nature can act as a good charge transport material in photovoltaic devices. In other words, their good electron accepting tendency and charge transport ability make them amenable towards fabricating photovoltaic devices with improved PCE. Besides, there are a few reports, which evinced that Cdots can act as an effective surface modifier, resulting in the lowering of energy barrier for electronic transportation. In those cases, we surmise that use of highly crystalline Cdots in place of Cdots with limited graphitic crystallinity might become helpful in increasing the PCE because of highly ordered geometry that is ideal for efficient charge transportation.

\subsection{Photocatalytic applications}

Solar energy conversion is not only an important way of meeting the demand of renewable energy but also an effective solution for reducing the use of polluting chemicals with the help of photocatalytic technology. Semiconducting nanomaterials such as $\mathrm{BiVO}_{4}, \mathrm{TiO}_{2}, \mathrm{ZnS}, \mathrm{ZnO}, \mathrm{Fe}_{2} \mathrm{O}_{3}$, CdS and GaP and Pt nanoparticles are traditionally used as catalysts for photocatalytic conversions. ${ }^{154-158}$ In addition, nowadays use of graphite, carbon nanotubes, $\mathrm{C}_{3} \mathrm{~N}_{4}$ and GQDs for efficient photocatalytic conversion is noteworthy. ${ }^{159}$ Of late, Cdots have been considered as a popular choice in the field of photocatalysis due to 
their strong absorption in the UV-vis region and PET properties. To date, plenty of synthetic strategies have been reported for the preparation of Cdot based efficient photocatalysts. In addition, semiconductor or metal based Cdot composites are found to have great potential to be used as the same.

Semiconductor materials are promising in the field of photocatalysis because of their broad light absorption. Cdots, being efficient in electron transfer and charge separation, are used with semiconductors in order to increase photocatalytic yields. Cdots integrated in $\mathrm{BiVO}_{4}$ Qdots performed as an excellent photocatalyst for water splitting. ${ }^{160} \mathrm{An} \mathrm{H}_{2}$ evolution rate of $0.92 \mu \mathrm{mol} \mathrm{h}^{-1}$ was achieved in the presence of $5 \% \mathrm{Cdot} /$ BiVO $_{4}$ Qdots, which was about 4 times that of only BiVO ${ }_{4}$ Qdots. The extended photon absorption range along with efficient charge separation following graphitic Cdot integration resulted in improved photocatalytic activity of the as-prepared composite system. ${ }^{160} \mathrm{TiO}_{2}$ based nanomaterials are extensively used semiconductors for solar energy conversion and photocatalytic applications. However, their wide bad gap $\left(E_{\mathrm{g}} \sim 3.2 \mathrm{eV}\right)$ with limited absorption in the UV region further restricts their potential applications. Various non-metal (such as B, C, N, and S) and transition metal (such as Fe, Pt, and Cr) dopants have been used therefore, to narrow the bandgap and achieve longer wavelength absorption. Wang and co-workers derived Cdots from L-ascorbic acid and introduced the same into $\mathrm{TiO}_{2}$ to improve visible light absorption. ${ }^{161}$ The as-prepared Cdot$\mathrm{TiO}_{2}$ displayed improved photocatalytic performance, which was 3.6 times higher than that of $\mathrm{TiO}_{2} \cdot{ }^{161}$ Similar to $\mathrm{TiO}_{2}$, the $\mathrm{ZnO}$ band gap is found to be $\sim 3.37 \mathrm{eV}$ and shows strong absorbance in the UV region. Therefore, in order to pursue visible light photocatalysis applications, Cdots with graphitic characteristics were used in the composite system to prepare an efficient photocatalyst. The photocatalytic efficiency for degradation of toxic gas was found to be $80 \% .{ }^{162}$ Unlike $\mathrm{TiO}_{2}$ or $\mathrm{ZnO}$, $\mathrm{Fe}_{2} \mathrm{O}_{3}$ has narrow band gap energy $(\sim 2.2 \mathrm{eV})$ and shows visible light absorption ( $<560 \mathrm{~nm})$. However, the observed catalytic efficiency for $\mathrm{Fe}_{2} \mathrm{O}_{3}$ remains low because of low charge carrier density and fast $\mathrm{e}^{-} / \mathrm{h}^{+}$pair recombination. Kang and co-workers fabricated an $\mathrm{Fe}_{2} \mathrm{O}_{3}$ /graphitic Cdot nanocomposite for improved photocatalytic degradation of toxic gases. ${ }^{163}$ Based on detailed analyses, they surmised that improvement in the efficiency was attributed to the presence of Cdots in the composite system. Graphitic Cdots have large electron-storage capacity, which therefore assists in shuttling of the photoexcited electrons from $\mathrm{Fe}_{2} \mathrm{O}_{3}$ particles through the conducting network inside carbon nanostructures. Next, electron-hole pairs react with $\mathrm{O}_{2}$ and $\mathrm{OH}^{-}$to produce active oxygen radicals (e.g. ${ }^{\bullet} \mathrm{O}^{2-}$ and ${ }^{\bullet} \mathrm{OH}$ ), which subsequently causes efficient degradation. ${ }^{163}$ Chai et al. reported a layer-by-layer fabrication method to prepare Cdot/CdS heterojunction films in order to control rapid $\mathrm{e}^{-} / \mathrm{h}^{+}$recombination. Cdots were prepared from L-cysteine via the pyrolysis method and were deposited over ITO substrates first. ${ }^{164}$ After that, plates were separately immersed in $0.05 \mathrm{M} \mathrm{CdCl}_{2}$ aqueous solution followed by $0.05 \mathrm{M} \mathrm{Na} \mathrm{Na}_{2} \mathrm{~S}$ aqueous solution for $30 \mathrm{~s}$ each. After repeating this cycle for 20 times, layered Cdot/CdS photoelectrodes were prepared.
Films showed strong photocatalytic reduction of nitro-aromatic compounds. Significant improvement in the photocatalytic activity was attributed to Cdots, which make intimate interfacial contact with CdS facilitating charge separation and transfer. The presence of Cdots in the composite allows visible light absorption leading to more photogenerated carriers. Further, Cdots act as electron sinks, accepting photoinduced electrons from CdS, thus improving the overall photocatalytic activity. ${ }^{164}$ Highly crystalline Cdot/Ag/ $/ \mathrm{Ag}_{3} \mathrm{PW}_{12} \mathrm{O}_{40}$ was prepared by Kang's group to improve visible light driven photocatalytic activities for water splitting and $\mathrm{H}_{2}$ evolution. ${ }^{165}$ An N doped Cdots $/ \mathrm{Bi}_{2} \mathrm{O}_{3}$ inverse opal structure (IOS) was prepared with improved photoelectrochemical and photocatalytic performance for rhodamine B degradation. ${ }^{166}$ The $\mathrm{N}$ doped $\mathrm{Cdot} / \mathrm{Bi}_{2} \mathrm{O}_{3}$ IOS exhibited a maximum photocurrent density of $0.75 \mathrm{~mA} \mathrm{~cm}^{-2}$, which was higher than those of Cdot $/ \mathrm{Bi}_{2} \mathrm{O}_{3}$ IOS $\left(0.63 \mathrm{~mA} \mathrm{~cm}^{-2}\right), \mathrm{Bi}_{2} \mathrm{O}_{3}$ IOS $\left(0.10 \mathrm{~mA} \mathrm{~cm}{ }^{-2}\right)$, and $\mathrm{Bi}_{2} \mathrm{O}_{3}$ NPs $\left(0.02 \mathrm{~mA} \mathrm{~cm}^{-2}\right) .{ }^{166}$ Observed enhancement of the photocurrent density was attributed to higher mobilization of photoinduced electrons from the photoanode to counter electrode under solar illumination. Here, excellent photoelectrochemical activity of $\mathrm{N}$ doped $\mathrm{Cdot} / \mathrm{Bi}_{2} \mathrm{O}_{3}$ IOS was because of sensitization of $\mathrm{N}$ doped Cdots and ordered porous structures. Co-catalysis plays a crucial role in enhancing the efficiency of semiconductor based photocatalysts during solar-light assisted water splitting. Gong's group reported the synergistic effect between well-crystallized graphitic Cdots and $\mathrm{Co}_{3} \mathrm{O}_{4}$, promoting photoelectrochemical water oxidation by an $\mathrm{Fe}_{2} \mathrm{O}_{3}$ photoanode. ${ }^{167}$ A photocurrent density of $1.48 \mathrm{~mA} \mathrm{~cm}{ }^{-2}$ at $1.23 \mathrm{~V}$ was achieved, which was found to be $78 \%$ higher than that of the $\mathrm{Fe}_{2} \mathrm{O}_{3}$ photoanode. Further, introduction of Cdots into ruthenium nanoparticles (Cdot@Ru composite), ${ }^{168}$ nickeliron layered double-hydroxide nanoplates (Cdot/NiFe-LDH) ${ }^{169}$ and $\mathrm{np}^{+}$-Si photocathodes ${ }^{170}$ for improving catalytic efficiency and $\mathrm{H}_{2}$ evolution rate is worth mentioning. Here, Cdots helped to improve the carrier transfer rate, electrochemical activity and flat band potential at the electrode-electrolyte interface. Also, due to their crystallinity and doping with heteroatoms such as nitrogen, electronic properties of neighbouring carbon atoms are modulated through intramolecular charge transfer, which is helpful in improving the hydrogen evolution rate.

In addition to Cdot/semiconductor nanocomposites, Cdots in combination with molecular catalysts are noteworthy. Martindale et al. used one-pot thermolysis of citric acid to prepare Cdots. ${ }^{1}$ The as-synthesized Cdots showed activity in the visible region beyond $\lambda>455 \mathrm{~nm}$ and was used as a photo-sensitizer in combination with $\mathrm{Ni}$ catalysts. Cdots directly transferred photoexcited electrons to molecular $\mathrm{Ni}$ catalysts, giving $398 \operatorname{mol}_{\mathrm{H}_{2}}\left(\mathrm{~g}_{\mathrm{Cdot}}\right)^{-1} \mathrm{~h}^{-1}$ activity along with a "per Ni catalyst" turnover frequency of $41 \mathrm{~h}^{-1} \cdot$ Also, functionalized carbon nanoparticles with gold or platinum were prepared for the photocatalytic conversion of $\mathrm{CO}_{2}$ to small organic acids. ${ }^{171,172}$

Preparation and introduction of metal free sustainable nanocomposite systems for efficient photocatalysis are worthy of mention. In the year 2015, Liu et al. reported a metal free Cdot-carbon nitride $\left(\mathrm{C}_{3} \mathrm{~N}_{4}\right)$ nanocomposite as an efficient catalyst for visible light driven water splitting. ${ }^{159}$ High-resolution 
TEM analysis showed the presence of a $0.202 \mathrm{~nm}$ interlayer spacing due to the (101) facet of Cdot crystallites. Linewidth analysis of powder XRD patterns corresponding to the Cdot$\mathrm{C}_{3} \mathrm{~N}_{4}$ composite suggested that the $\mathrm{C}_{3} \mathrm{~N}_{4}$ matrix contained nanocrystallites with $4 \mathrm{~nm}$ of average diameter. Measured quantum efficiencies were $16 \%$ for $\lambda=420 \pm 20 \mathrm{~nm}, 6.29 \%$ for $\lambda=580 \pm 15 \mathrm{~nm}$ and $4.42 \%$ for $\lambda=600 \pm 10 \mathrm{~nm}$, along with $2.0 \%$ overall solar energy conversion efficiency. In contrast to the established one-step four-electron pathway, the Cdot- $\mathrm{C}_{3} \mathrm{~N}_{4}$ system was found to catalyse water splitting via a two-electron two-step process. $\mathrm{C}_{3} \mathrm{~N}_{4}$ was responsible for photocatalysis in the first step, whereas Cdots were responsible for chemical catalysis in the second step. Also, Cdots increased the light absorbance of the composite. Moreover, Cdot $-\mathrm{C}_{3} \mathrm{~N}_{4}$ retained a significant hydrogen and oxygen evolution rate with stability up to 200 runs along with recycling use over 200 days. ${ }^{159}$

Apart from nanocomposite systems, there are also reports showing pristine Cdots as efficient photocatalysts. Li et al. prepared Cdots through an alkali-assisted electrochemical method by using graphite rods as both cathode and anode. ${ }^{173}$ The as-prepared Cdots had distinct crystal structures with graphitic fragments and showed size dependent upconversion PL. Using NIR light driven photo-induced electron transfer properties of crystalline Cdots, metal free photocatalytic oxidation of benzyl alcohol into benzaldehyde was demonstrated. Notably, the conversion efficiency was found to be $92 \%$ with $100 \%$ selectivity under NIR irradiation. ${ }^{173}$ They also prepared Cdots via electrochemical ablation of graphite, which were used as visible light-induced acid photocatalysts in aqueous solution. The catalytic activity was attributed to efficient visible light-induced proton generating ability due to the oxygen-containing surface functional groups attached to graphitic networks. ${ }^{174} \mathrm{Hu}$ et al. reported preparation of Cdots using chemical oxidation of activated carbon powder and soot, demonstrating that photocatalytic activities of Cdots can be improved significantly by tuning their surface states. ${ }^{175}$ Indeed, oxygen-containing surface functional groups affect the separation and recombination of the electron-hole pairs. Surface functional groups, such as the $-\mathrm{C}=\mathrm{O}$ and $-\mathrm{COOH}$, facilitate effective separation of electron-hole pairs increasing the photocatalytic activity. On the other hand, reduced surface states related to $-\mathrm{C}-\mathrm{OH}$ and $-\mathrm{CHNR}$ prevent the electron-hole pairs from surface recombination, decreasing the efficiency. ${ }^{175}$ Additional studies showed the effect of structure and morphologies of Cdots on photocatalytic activity, based on the photoreduction of methyl viologen $\left(\mathrm{MV}^{2+}\right) .{ }^{176}$ In this study they have proved that graphitic non-doped Cdots have high photocatalytic activity as compared to amorphous non-doped Cdots on the basis of $\mathrm{MV}^{2+}$ photoreduction. $\mathrm{N}$ doped Cdots were synthesized through a one-step hydrothermal method, which served as an excellent photocatalyst for methylene blue dye degradation under sunlight. Catalytic degradation, in this work, was proposed to follow pseudo-first order kinetics according to the Langmuir-Hinshelwood model. ${ }^{177}$

To summarize, efficient photocatalytic activities by Cdots are not only attributed to broad visible light absorption leading to photogenerated carriers but are also dependent on efficient electron transfer and charge separation ability and good electron accepting tendency from semiconductors and various surface functional groups. The reports, discussed above, are based on Cdots having short-range graphitic structures inside. Although reports on the presence of exceptionally high nongraphitic macro-crystallinity in Cdots are very recent, such high crystalline domains in Cdot structures could be valuable for efficient electron transfer and charge separation leading to efficient photocatalysis.

\subsection{Optoelectronic devices}

Light-emitting diodes (LEDs) are portable solid-state devices that generate light using semiconductors. LEDs are of substantial research interest in the present days because of their potential utilization in full-color display technology, fabricating liquidcrystal displays and white LEDs (WLEDs). In this regard, as an emerging class of "nanolights" Cdots appear to be useful alternatives to toxic, heavy metal based semiconducting quantum dots and rare earth metal-based high-cost phosphors. Large optical bandwidths along with tunable optical band gaps have made Cdots viable for their utilization in energy-saving technologies, i.e., fabricating portable devices having low power consumption, long functioning time and low maintenance cost.

The utilization of Cdots in fabricating LED devices can broadly be categorized into two classes. One is optical excitation, where Cdots with a graphitic core are used as the phosphor components resulting in full colour emission from LEDs. The other one corresponds to electrical excitation of such Cdots resulting in electroluminescence (EL) from the Cdot active emission layer.

As mentioned in the previous section, LEDs based on the PL properties of Cdots mainly consist of "UV-LED chips" along with Cdots as the phosphor material, which can be excited by the strong blue emission from the "chips". In the year 2012, Guo et al. reported synthesis of multi-colour fluorescent Cdots via chemical unzipping of polystyrene photonic crystals at different pyrolysis temperatures. ${ }^{178}$ Well-resolved crystalline planes such as (100), (020), and (002) corresponding to graphitic facets were detected. Finally, they applied the as-prepared multi-colour emitting solid Cdots (blue, orange and white) in fabricating LED devices using a $370 \mathrm{~nm}$ excitation source. Notably, warm white light emission with colour coordinates of $(0.34,0.37)$ was achieved by the group. Later in 2013 , Chen et al. synthesized Cdots having broad yellow fluorescence by following one-step pyrolysis of $N$-acetylcysteine. ${ }^{179}$ After that, they fabricated white-light emitting diodes by combining yellow emitting as-prepared Cdots and blue emitting GaN-based "LED chips". The LEDs exhibited warm white light emission with the calculated colour coordinates of $(0.34,0.35)$, which are close to perfect white light emission. However, remarkable PL quenching due to aggregation in solid states hampers Cdot applications in fabricating LED devices. It is believed that drying of Cdot samples following synthesis results in interactions among different surface states (caused by various surface functional groups), which facilitates solid state PL quenching in the end, 
similar to organic fluorescent dyes. In order to avoid such problems, a few research groups have come up with new ideas of embedding Cdots in different solid matrices such as starch,${ }^{180}$ polymers, ${ }^{48,181}$ cellulose nanofibers, ${ }^{182}$ silicone ${ }^{183}$ and borax, ${ }^{184}$ which prevents self-quenching of Cdot emission. For example, Sun et al. prepared Cdot/starch composites to fabricate high QY WLEDs (QY of $\sim 50 \%$ ). ${ }^{180}$ Rhee and co-workers used a polymethyl methacrylate (PMMA) matrix in order to embed nitrogen doped carbon nanodots and then excited the polymer encapsulated Cdot phosphor with InGaN UV light-emitting diodes. ${ }^{181}$ By supplying a forward current of $50 \mathrm{~mA}$ bright warm-white light emission was achieved with colour coordinates of $(0.37,0.41)$ and correlated colour temperature (CCT) of $4385 \mathrm{~K}$. In these cases, QY of the phosphor material is an important factor. The presence of a higher amount of $\mathrm{sp}^{2}$ clusters helps in delocalization of lone pair electrons of the heteroatoms resulting in effective reduction in energy gap between the HOMO and LUMO. Such a conjugation not only results in a red-shift in emission maxima but also increases QY. Radiative recombination rate constant values $\left(K_{\mathrm{r}}\right)$ are found to be higher than non-radiative recombination rate constant values $\left(K_{\mathrm{nr}}\right)$. Thus, non-radiative relaxation is minimized and phosphor emission is intensified.

It is important mentioning here that high luminescence efficiency along with an appropriate color rendering index (CRI) and correlated color temperatures (CCT) are the important parameters for fabricating quality WLEDs, which are still difficult to achieve in the case of Cdots due to low QY in the longer wavelength region. Also, the obtained efficiencies are found to be quite low as compared to well-developed semiconducting QD (such as CdSe, CdTe and PbTe) LEDs. Thus, new techniques are required for the large-scale synthesis of Cdots with high QY in the longer wavelength region. Besides, a recent report suggests that bright white-light emission from Cdots at the single particle level can be achieved by synthesizing them using one step microwave treatment of glycerol, $p$-phenylenediamine and $o$-phosphoric acid. ${ }^{48}$ The as-synthesized Cdots consisted of $0.23 \mathrm{~nm}$ lattice spacing due to graphitic carbon. They were found to be retaining pure bright white-light emission capability even when they were encapsulated inside a polyvinyl alcohol (PVA) solid matrix. Calculated CCT and CRI values were found to be $8442 \mathrm{~K}$ and 74 respectively (colour coordinates $(0.29,0.33)$ at $340 \mathrm{~nm}$ excitation), which are much higher as compared to previous reports. ${ }^{48}$ Such techniques of synthesizing Cdots could be effective during the fabrication of efficient phosphor based WLED devices.

In the case of EL based LEDs, Cdot emission layers are sandwiched between charge transport layers. In the year 2011, Gupta and co-workers first reported graphene QD (GQD) based organic LEDs (OLEDs) ${ }^{132}$ In this work, methylene blue functionalized GQDs were doped in poly(2-methoxy-5-(2-ethylhexyloxy)-1,4-phenylenevinylene) (MEH-PPV) with different concentrations and then they were tested as active emission layers for OLED application. It was observed that the light emission efficiency of MEH-PPV could be improved significantly following addition of methylene blue functionalized
GQDs to the same. ${ }^{132}$ The turn-on voltages were found to be $6 \mathrm{~V}$ with pure MEH-PPV and $4 \mathrm{~V}$ for $1 \%$ methylene blue functionalized GQDs in MEH-PPV. Addition of methylene blue functionalized GQDs allowed improved electrical transportation and hence better charge injection and higher carrier density. This resulted in lower turn-on voltage and improved efficiency. Later on, 1-hexadecylamine passivated Cdots were used for successful fabrication of WLEDs based on EL (Fig. 13a-e). ${ }^{185}$ Briefly, a Cdot active emission layer ( $20 \mathrm{~nm}$ thick) was spun-cast between a 1,3,5-tris( $N$-phenylbenzimidazol-2-yl) benzene (TPBI) layer (electron-transport layer; $40 \mathrm{~nm}$ thick) and a poly(3,4ethylenedioxythiophene):poly(styrene sulfonate) (PEDOT:PSS) layer (hole injection layer; $40 \mathrm{~nm}$ thick). Color coordinates of $(0.40,0.43)$ and a CRI of 82 were achieved along with a maximum brightness of $35 \mathrm{~cd} \mathrm{~m}^{-2}$ and $0.083 \%$ external quantum efficiency (EQE) in this work. ${ }^{185}$ Recently, a maximum luminescence $\left(L_{\max }\right)$ of $2050 \mathrm{~cd} \mathrm{~m}^{-2}$ was achieved at $3.9 \mathrm{~V}$ turn-on voltage for Cdot based WLEDs. ${ }^{98}$ In another report, carbon quantum dots having narrow bandwidth emission were prepared from solvothermal treatment of phloroglucinol by Yuan et al. with $L_{\max }$ ranging between $1882-4762 \mathrm{~cd} \mathrm{~m}^{-2}$ and a current efficiency of 1.22$5.11 \mathrm{~cd} \mathrm{~A}^{-1} \cdot{ }^{186}$ Unique crystalline triangular geometry along with electron-donating hydroxyl surface-groups resulted in highly delocalized charges and good structural stability, which was eventually found to assist in reduced electron-phonon coupling, liable for color-purity excitonic emission. Oleophilic graphitic Cdots with $41 \%$ QY were synthesized from citric acid and hexadecylamine via microwave treatment. ${ }^{187}$ Subsequently, Cdot electroluminescence was used to prepare bright LEDs (Fig. 13f). In this work, $\mathrm{Xu}$ et al. prepared a Cdot doped poly( $N$-vinylcarbazole) (PVK) based "host-guest" system, acting as the emitting layer. Bright white and yellow emissions were obtained from the as-prepared devices by tuning the structure and doping concentrations. The white LED exhibited high luminance $\left(455.2 \mathrm{~cd} \mathrm{~m}^{-2}\right)$ and CRI (83) along with obtained CIE coordinates of $(0.29,0.33)$. The yellow LED, on the other hand, showed a maximum brightness of $339.5 \mathrm{~cd} \mathrm{~m}^{-2} \cdot 187$

However, Cdot EL based LED fabrication is still at the initial stage and requires more in-depth research. Key challenges during device fabrication are the low QY of Cdots in the solid state and relatively weak carrier transport properties (hole and electron transportation) due to poor structural arrangement (i.e., poor crystalline nature or partial graphitization). Precisely, optimization of structural alignment is necessary to achieve sufficient charge carrier transport ability. Also, much attention is required for energy level matching with the electron transport layer and hole transport layer by tuning thickness, while fabricating commercially available devices.

\subsection{Energy storage applications}

In the context of ever-increasing energy demand and call for the development of green and sustainable energy resources, electrochemical energy storage devices have drawn tremendous attention because of their low-cost fabrication, high energy and power density, rapid charging-discharging ability, and low maintenance cost. ${ }^{188}$ Batteries and capacitors are two types 
(a)

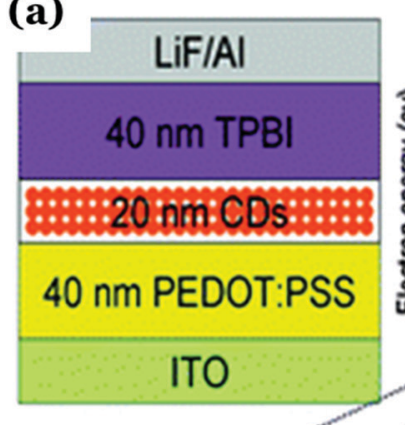

(c)

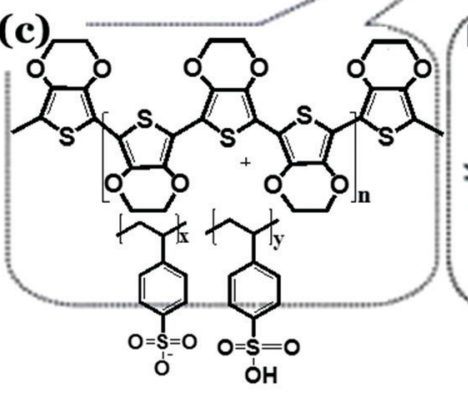

(b)

(f)
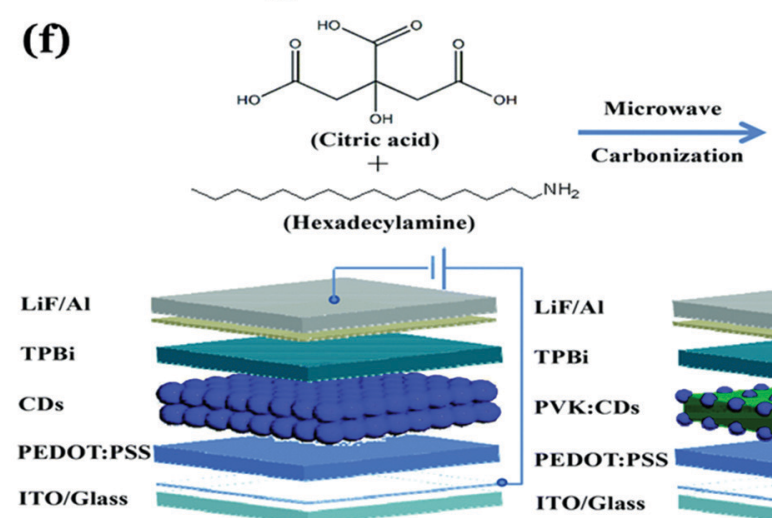

CD-LED (non-doped, Device-1)

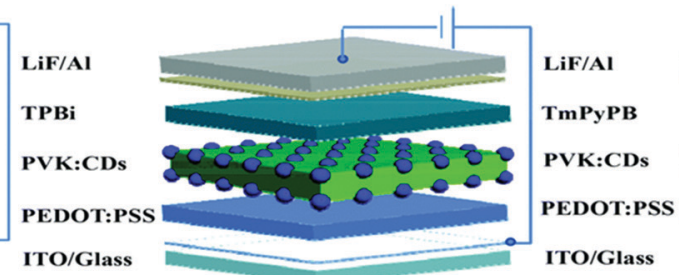

White CD-LEDs (doped, Device-2) (d)
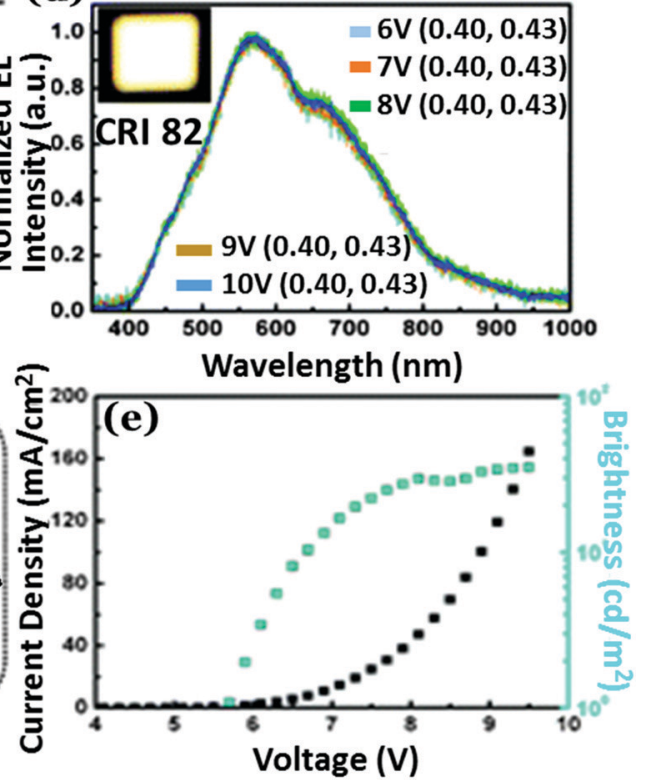

Yellow CD-LEDs (doped, Device-3)

Fig. 13 (a) Schematic representation of the Cdot based WLEDs. (b) Energy level diagram of WLEDs relative to ITO and LiF/Al work function and (c) the molecular structures of the layers. (d) Normalized electroluminescence spectra of WLEDs. (e) Current density, brightness vs. voltage characteristic plots recorded for WLEDs. Reprinted with permission ref. 185. Copyright 2011 Royal Society of Chemistry. (f) Illustration for the synthesis of oleophilic Cdots and the LED devices fabricated with the as-prepared Cdots. Reprinted with permission ref. 187. Copyright 2018 Royal Society of Chemistry.

of commonly used energy storage devices. ${ }^{189}$ In effect, batteries are found to have higher energy density but limited power density whereas capacitors can have higher power density and lower energy density. ${ }^{189}$ Of late, researchers have come up with the solution to overcome the aforementioned issues by building supercapacitors. Supercapacitors are electrochemical capacitors having reasonable energy density and high-power density along with remarkable charge storage and cycling stability. ${ }^{188,190}$ Apart from various semiconductor Qdots, ${ }^{191,192}$ metal oxide nanoparticles and composite systems, ${ }^{193,194}$ use of carbonaceous materials such as graphene, CNTs, activated carbon, carbon nanospheres, carbon foam, carbon nanosheets and fullerene is popular for electrode preparation for the same. ${ }^{195-201}$ To further optimize the efficiency of batteries and capacitors or supercapacitors, researchers have recently used Cdots to prepare low-cost, sustainable, high capacity and long lifecycle electrodes for energy harvesting. Use of a Cdot system in building energy storage devices and contribution of its crystalline nature in increasing the overall device performance are detailed in the following sections.

Batteries. Lithium-ion or sodium-ion batteries (LIBs or SIBs) correspond to rechargeable cells where lithium or sodium ions act as potential charge carriers. ${ }^{202}$ Use of carbonaceous materials derived from various resources is popular in fabricating efficient electrodes for LIBs or SIBs. However, one of the most critical issues for the construction of high-performance batteries is to find suitable electrode materials. Recent literature suggests that Cdots can be applied for structural alterations of the active electrode material resulting in improved performance. ${ }^{32}$ For example, Jing et al. reported synthesis of a Cdot coated $\mathrm{Mn}_{3} \mathrm{O}_{4}$ composite using an alternating voltage electrochemical approach. ${ }^{203}$ The as-prepared $\operatorname{Cdot} / \mathrm{Mn}_{3} \mathrm{O}_{4}$ nanocomposite was used as an anode material for LIBs, demonstrating efficient electrochemical performances, with a 
measured discharge capacity of $934 \mathrm{~mA} \mathrm{~h} \mathrm{~g}^{-1}$ after 50 cycles at $100 \mathrm{~mA} \mathrm{~g}^{-1}$ current density. Notably, the measured values were nearly five times higher as compared to the same for a $\mathrm{Mn}_{3} \mathrm{O}_{4}$ electrode. In the composite, generation of the Cdot induced octahedral structure of $\mathrm{Mn}_{3} \mathrm{O}_{4}$ was beneficial, which reduced charge transfer resistance due to alternating $\mathrm{Mn}$ and $\mathrm{O}$ atom layer structures, resulting in higher coulombic efficiency. Moreover, powder XRD of Cdot/ $\mathrm{Mn}_{3} \mathrm{O}_{4}$ confirmed narrower diffraction peaks due to lowering in lattice parameter values following Cdot incorporation into $\mathrm{Mn}_{3} \mathrm{O}_{4} \cdot{ }^{203}$ Use of hybrid phase nanocomposites such as $\mathrm{Li}_{2} \mathrm{NaV}_{2}\left(\mathrm{PO}_{4}\right)_{3} / \mathrm{Cdot}$ as the cathode material in low-cost and efficient LIBs is noteworthy. ${ }^{204}$ Wang et al. prepared a $\mathrm{Li}_{2} \mathrm{NaV}_{2}\left(\mathrm{PO}_{4}\right)_{3} / \mathrm{Cdot}$ (H-LNVP/Cdot) composite system using the sol-gel and thermal reduction technique. The graphitic nature of the Cdots over H-LNVP was confirmed via Raman spectroscopy. A relatively lower $I_{\mathrm{D}} / I_{\mathrm{G}}$ ratio $(0.61)$ indicated the presence of higher degree of graphitic carbon, which finally resulted in enhanced electrical conductivity. The composite cathode material showed a discharge capacity of $158 \mathrm{~mA} \mathrm{~h} \mathrm{~g}{ }^{-1}$ and could retain $97.6 \%$ of measured capacity after 50 cycles of use. Cdots have excellent charge transfer rates along with good electron transport efficiency due to quantum tunnelling, which results in improved efficiency. ${ }^{204}$ A new type of Cdot coated $\mathrm{VO}_{2}$ nanowire grown over $3 \mathrm{D}$ carbon cloth was prepared via a surface engineering process and was introduced as a cathode material to increase the performance of LIBs and SIBs. In this work, Cdots were synthesized from sucrose using the hydrothermal process and they played a crucial role in free electron diffusion. ${ }^{205}$ High-resolution TEM confirmed well resolved lattice distances of 0.21 and $0.20 \mathrm{~nm}$, corresponding to the $P 63 / \mathrm{mmc}$ hexagonal planes of carbon. $\mathrm{VO}_{2}$ nanowires coated with continuous layers of crystalline Cdots enhanced the overall conductivity. ${ }^{205}$ Cdots can be used to derive 3D porous carbon frameworks (PCFs) through the high temperature calcination technique. ${ }^{206}$ The as prepared PCFs consisted of thin carbon films with $0.42 \mathrm{~nm}$ interlayer spacing, providing more sodium storage sites and perfect sodium ion diffusion length. The initial charge capacity was found to be $104.1 \mathrm{~mA} \mathrm{~h} \mathrm{~g}^{-1}$ at $5 \mathrm{~A} \mathrm{~g}^{-1}$ current density and showed $99.8 \mathrm{~mA} \mathrm{~h} \mathrm{~g}^{-1}$ capacity after 10000 cycles of use. ${ }^{206}$ These values were much higher as compared to several other reports. $\mathrm{N}$ doped $\mathrm{TiO}_{2}$ nanorods decorated with Cdots were fabricated using hydrothermal treatment of $\mathrm{TiO}_{2}$ powders (P25) and $\mathrm{NaOH}$ in the presence of Cdots. ${ }^{207}$ The $\mathrm{N}-\mathrm{TiO}_{2} / \mathrm{Cdot}$ composite showed intense sharp peaks in its diffraction pattern. Ion exchange followed by calcination finally led to the formation of a nanocomposite system with enhanced electrical-conductivity and rapid chargetransfer properties. The as-prepared composite was successfully used as an anode material and showed a capacity of $185 \mathrm{~mA} \mathrm{~h} \mathrm{~g}^{-1}$ with $91.6 \%$ capacity retention for over 1000 cycles. The overall performance of the $\mathrm{N}-\mathrm{TiO}_{2} / \mathrm{Cdot}$ composite electrode was far better than that of $\mathrm{N}-\mathrm{TiO}_{2}$ in both the LIB and SIB. Conductivity of the $\mathrm{N}-\mathrm{TiO}_{2} / \mathrm{Cdot}$ composite film was found to be about ten times more than that of the $\mathrm{N}-\mathrm{TiO}_{2}$ film $\left(5.1 \times 10^{-7} \mathrm{~S} \mathrm{~cm}^{-1}\right.$ for composite and $5.31 \times 10^{-8} \mathrm{~S} \mathrm{~cm}^{-1}$ for pure $\mathrm{N}-\mathrm{TiO}_{2}$ film). Further, electrochemical impedance spectroscopy (EIS) studies confirmed lowering in charge transfer resistance in both LIB and SIB due to the presence of Cdots. ${ }^{207}$

Supercapacitors. Supercapacitors are defined as charge storage devices that store energy either electrostatically or electrochemically. Corresponding devices are known as electric double-layer capacitors (EDLCs) and pseudocapacitors, respectively. In fact, the charge storage capacity of pseudocapacitors is quite a lot higher than that of EDLCs since they follow the faradaic charge storage mechanism. The first reported electrochemical capacitor was made of a porous carbon-based electrode in the year $1957 . .^{208}$ Until now, various inorganic nanostructured materials such as QDs, perovskites, metal oxide nanoparticles, nanowires and metal-organic frameworks (MOFs) have been applied to fabricate electrodes for energy storage devices. ${ }^{209-213}$ However, as far as green and sustainable high-capacity energy storage devices are concerned fabrication of carbonaceous material-based electrodes for ultracapacitors are worth mentioning. So far, the major drawback for inorganic electrodes is poor cycle-life and for carbon-based electrodes, low capacitance is the limitation. In order to address these issues nanohybrid material-based electrodes have been prepared. Current research on energy storage devices is focused on developing novel electrodes having high energy and power densities, large specific capacitance and long cycle life. In this regard, Cdots have drawn attention towards improving electrochemical performance of electrodes due to their large surface area, good conductivity as well as structural flexibility. Cdotmetal oxide nanohybrids, porous materials and Cdot-polymer nanocomposites have been widely used for capacitors.

Introduction of Cdots into hybrid materials creates a conducting network facilitating rapid ion transportation during the charge-discharge process. ${ }^{214}$ Cdots, synthesized via chemical oxidation of graphite, was decorated with $\mathrm{RuO}_{2}$ to prepare a nanocomposite system. Remarkably, the composite material exhibited a specific capacitance of $460 \mathrm{~F} \mathrm{~g}^{-1}$ when the current density was $50 \mathrm{~A} \mathrm{~g}^{-1} \cdot{ }^{214}$ Introduction of Cdots greatly improved the charge-discharge ability of $\mathrm{RuO}_{2}$. Here, graphitic nature of Cdots played a crucial role for fast ionic motion and charge transportation by generating highly conducting channels through the hybrid network structure. Zhu et al. prepared a Cdot doped porous $\mathrm{NiCo}_{2} \mathrm{O}_{4}$ composite via a reflux synthesis route. ${ }^{215}$ The as prepared $\mathrm{Cdot} / \mathrm{NiCo}_{2} \mathrm{O}_{4}$ composite material with characteristic diffraction peaks was used to build an asymmetric supercapacitor with a high energy density of $27.8 \mathrm{~W} \mathrm{~h} \mathrm{~kg}{ }^{-1}$ at $128 \mathrm{~W} \mathrm{~kg}^{-1}$ power density. The composite electrode showed a high specific capacitance of $856 \mathrm{~F} \mathrm{~g}^{-1}$ at $1 \mathrm{~A} \mathrm{~g}^{-1}$ current density and could retain up to $98.75 \%$ of the initial capacitance after 10000 cycles of use at $5 \mathrm{~A} \mathrm{~g}^{-1} \cdot{ }^{215}$ The good electrochemical performance by the $\mathrm{Cdot} / \mathrm{NiCo}_{2} \mathrm{O}_{4}$ composite was ascribed to the large specific surface area along with high conductivity and porous structure, beneficial for facile electrolyte transportation. ${ }^{215}$ Huang's group demonstrated Cdot mediated synthesis of $\mathrm{MnO}_{x}$ and further preparation of a $\mathrm{MnO}_{x}$-graphene composite system as an efficient electrode material. ${ }^{216}$ The fabricated solid-state supercapacitor exhibited a specific capacitance of $\sim 280 \mathrm{~F} \mathrm{~g}^{-1}$ at $1 \mathrm{~A} \mathrm{~g}^{-1}$ current density 
along with cyclic stability $>10000$ cycles. ${ }^{216}$ Additionally, reports on Cdot based $\mathrm{Ni}-\mathrm{Al}$ layered double hydroxide, ${ }^{217} \mathrm{NiO}$ nanorod composites $^{218}$ and CuS/carbon nanospheres ${ }^{219}$ showed remarkable improvement in electrochemical performances of the capacitors induced by crystalline graphitic Cdots.

The porous morphology of the electrode materials assists in achieving good specific capacitance. Embedding crystalline Cdots in such porous structures is found to increase capacitor performance either by providing a conductive network for ion transportation or by accumulating higher concentrations of counter ions due to higher surface charge. Activated carbons are widely used as electrode materials because of their large surface area and high porosity. However, low conductivity and amorphous nature limit their performances. Porat and co-workers showed that the specific capacitance of activated carbon could be improved up to three times by embedding Cdots in the porous structure. The strong electronegative nature of Cdots assisted in effective charge separation and counter ion accumulation outside the pores, resulting in improved electrochemical performance. Their structural alignment assisted in facile ionic motion as well. ${ }^{20}$ A porous 3D Cdot embedded aerogel was prepared by assembling Cdots during the sol-gel polymerization of resorcinol with formaldehyde. A supercapacitor fabricated with the Cdot-aerogel exhibited a 20 -fold increase in specific capacitance as compared to the Cdot-free aerogel. ${ }^{221}$

Conducting polymers are commonly used as electrode materials in pseudocapacitors. It has been observed that introduction of Cdots into polymers results in better electrochemical performance by the electrodes. For example, a polyaniline/Cdots (PANI/Cdots) nanocomposite was prepared using an oxidative polymerization method, which showed high stability and enhanced specific capacitance due to Cdots. Charge transfer resistance was found to be reduced considerably following Cdot integration inside the composite structure. ${ }^{222}$ Indeed, the graphitized structure of Cdots helped in improved electron transfer among neighbouring polymer networks. Zhang et al. reported a ternary composite of graphene oxide/carbon dot/polypyrrole (GO/Cdot/PPy) as an active material for supercapacitors. ${ }^{223}$ In that work, ultra-small conductive Cdots were sandwiched between the GO film and PPy layer so that Cdots can facilitate electron transportation, thus reducing the internal resistance as well as charge transfer resistance of the as-prepared electrode. ${ }^{223}$

Instead of optimizing the surface area and pore structures of the carbonaceous materials, Xiong and co-workers focused on constructing electron-rich regions on the electrode surfaces in order to trap more cations and improve the charge transfer process. ${ }^{224}$ They prepared porous carbon materials via calcination of Cdothydrogel composites, having abundant electron-rich surface defects due to phosphate/nitrogen containing groups from Cdots. Results showed that due to the presence of such electron-rich pockets over the electrode surface the capacitor performance could be increased significantly (Fig. 14a-c). ${ }^{224}$ Such electron pockets efficiently adsorb more cations and stimulate the charge transport process.

Cdots have been applied to build micro-supercapacitors as well. For example, Cdot/graphene microfibers were used to fabricate high-performance micro-supercapacitors. ${ }^{225}$ Cdots serve as reinforcing nanofillers resulting in a higher mechanical strength of $109.93 \mathrm{MPa}$. Also, integration of Cdots resulted in enhanced specific surface area of the composite from $245.6 \mathrm{~m}^{2} \mathrm{~g}^{-1}$ to $435.1 \mathrm{~m}^{2} \mathrm{~g}^{-1}$. TEM images confirmed that Cdots assisted in anchoring adjacent graphene sheets and due to the formation of such dot-sheet structures restacking of sheets was prevented. The constructed device thus delivered a high capacitance of $607 \mathrm{mF} \mathrm{cm}^{-2}$, cycling stability up to 10000 cycles and long-term bending durability. ${ }^{225}$ Polycrystalline Cdots were self-assembled to a layer structure by freeze-drying. By packaging such Cdot assembly with carbon black in electrodes, a volumetric capacitance of $157.4 \mathrm{~F} \mathrm{~cm}^{-3}$ and an areal capacitance of $0.66 \mathrm{~F} \mathrm{~cm}^{-2}$ were achieved. $^{226}$ It is important to note that, in most of the previous studies, Cdots had very limited graphitic crystallinity. We believe that instead of using such partially graphitized carbon cores, if we are able to generate macro-crystallinity in carbon nanostructures and use them in supercapacitors then efficiencies can be improved significantly. Recent techniques used to prepare highly crystalline Cdots for EDL capacitors are significant in this regard. In fact, it has been found that enhanced crystallinity in Cdots can effectively improve the electrochemical performance of electrodes. A phosphorus doped crystalline Cdot (P-Cdots) embedded activated carbon composite was prepared to develop efficient EDL capacitors. It was found that the integration of such crystalline Cdots could result in good electrochemical performance of the electrodes. For the activated carbon (AC)/P-Cdot electrode, the measured specific capacitance was found to be $263.6 \mathrm{~F} \mathrm{~g} \mathrm{~g}^{-1}$ whereas the same for the activated carbon electrode was $144.3 \mathrm{~F} \mathrm{~g}^{-1} \cdot{ }^{128} \mathrm{Next}$, zinc ion induced assembly in crystalline Cdots (Zn, P-Cdots) was generated in order to further improve the crystal quality. Interestingly, the specific capacitance corresponding to the AC/Zn, P-Cdot electrode was found to be $743.2 \mathrm{~F} \mathrm{~g}^{-1}$ along with $87.5 \%$ retention even after 4000 cycles of use (Fig. 14d-h). Detailed analyses confirmed that, instead of large surface area or high porosity, electrochemical performances were dependent on the extent of crystallinity. High crystallinity in the assembled Cdot structure created conductive channels through the micropores of activated carbon, which assisted in facile electrolyte transportation, thus enhancing the capacitive behaviour. ${ }^{128}$

\subsection{Sensing application of Cdots}

Because of excellent optical properties, ${ }^{227}$ Cdots have been extensively investigated as sensors in the field of fluorometric detection. Addition of analytes leads to quenching/enhancement in the PL intensity following interaction with Cdots. In this regard, development of Cdot-FRET or PET based ratiometric sensors are noteworthy. ${ }^{228,229}$ Until now, tremendous efforts have been devoted to the large-scale synthesis of Cdots by following cost effective techniques. Low toxicity, good biocompatibility, high QY and stable optical properties make Cdots promising fluorescent probes as compared to organic dyes or semiconducting QDs. ${ }^{14,96}$ Fluorescence based sensing techniques using Cdots can be broadly categorized into two types such as the solution phase detection and solid phase 
(a)

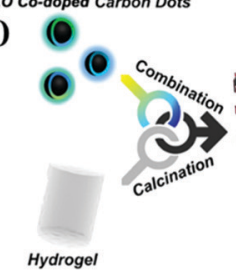

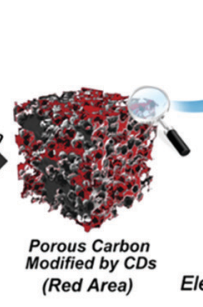
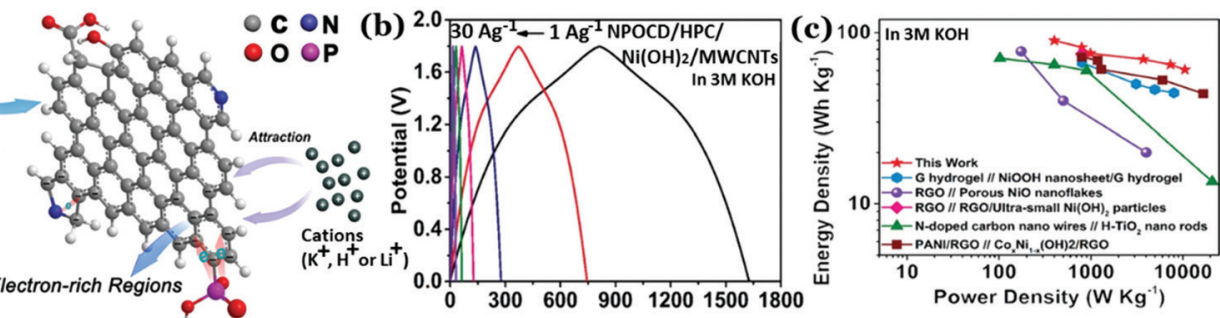

(d)
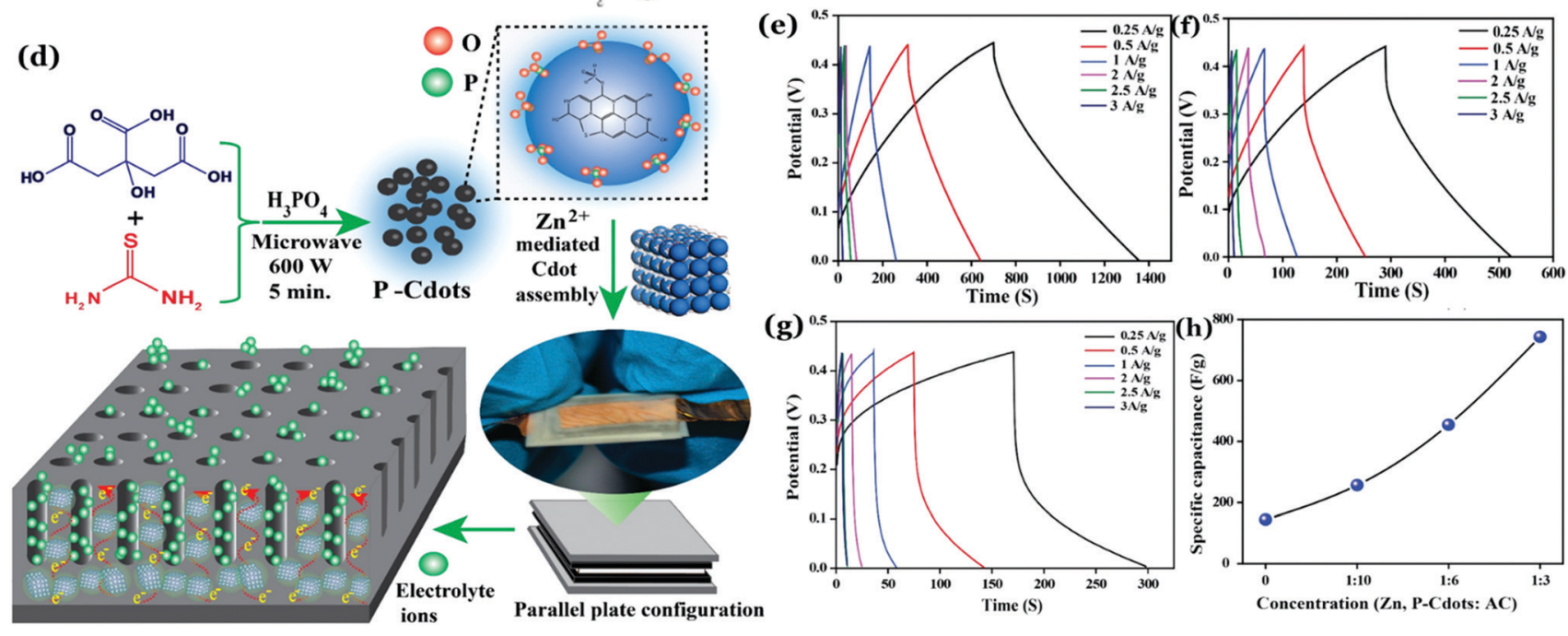

Fig. 14 (a) Porous carbon derived from a Cdot-hydrogel. (b) Galvanostatic charge-discharge (GCD) curves corresponding to hybrid supercapacitors at different current densities under alkaline conditions. (c) Ragone plots of the devices. Reprinted with permission from ref. 224. Copyright 2019 John Wiley and Sons. (d) Schematic corresponding to crystalline Cdot induced improved electrochemical performance by the electrodes. GCD plots for (e) Zn, $\mathrm{P}-\mathrm{Cdot} / \mathrm{AC}$, (f) P-Cdot/AC and (g) activated carbon electrode based EDLCs. (h) Increase in specific capacitance of the SC devices with increasing Zn, $\mathrm{P}-\mathrm{C}$ dot loading concentration into activated carbon. Reprinted with permission from ref. 128. Copyright 2019 American Chemical Society.

detection. The solution phase detection technique has been used to sense various analytes such as small molecules, intracellular $\mathrm{pH}$, macromolecules, cations, anions and moisture content. ${ }^{16,79,230-236}$ Solid phase detection, on the other hand, corresponds to utilization of fluorescent paper based or fingerprint-based sensing of various biomolecules, explosive compounds etc. ${ }^{16,237,238}$

Apart from colorimetric detection, recent reports on the use of Cdots as an electrochemical sensing platform are worthy of mention. In comparison with conventional fluorescence-based detection, electrochemical methods are more convenient because they are inexpensive, highly selective and sensitive, and easy to fabricate and miniaturize. In electrochemical sensors, electrodes are used to detect changes associated with electrochemical reactions due to interactions between analytes and the sensing surface. Organic polymers and nanocarbon systems are quite popular in fabricating working electrodes for such devices. Apart from the working electrode, reference electrodes and, often, counter electrodes are used during the measurements. Recently, Cdots have been applied to improve the sensitivity of working electrodes in electrochemical sensors. First, Cdots were synthesized from sodium citrate and $\mathrm{NH}_{4} \mathrm{HCO}_{3}$ via a hydrothermal process. ${ }^{239}$ After that, Cdots and $\mathrm{CoFe}$ layered double hydroxide (LDH) composite was immobilized with horse radish peroxidase (HRP) on a glassy carbon (GC) electrode. Sufficient crystallinity was detected in powder XRD analysis. Due to the synergistic effect among crystalline CoFe-LDHs, Cdots and HRP fast electron transfer behaviour was observed, resulting in electrocatalytic reduction of $\mathrm{H}_{2} \mathrm{O}_{2}$. For HRP/Cdots/LDHs/GCE, LOD for $\mathrm{H}_{2} \mathrm{O}_{2}$ was calculated to be $0.04 \mu \mathrm{M}$, much better than that of LDHs/GCE (LOD = $0.68 \mu \mathrm{M}){ }^{239}$ The Cdot infused $\mathrm{g}-\mathrm{C}_{3} \mathrm{~N}_{4}$ heterostructure showed sharp diffraction peaks in XRD along with well-resolved facets in high-resolution TEM images. ${ }^{240}$ It has been found that integration of Cdots into g- $\mathrm{C}_{3} \mathrm{~N}_{4}$ promotes efficient electron/ hole separation, resulting in a strong photocurrent in the heterostructure. ${ }^{20}$ Further addition of copper nanocluster (CuNC) functionalized prostate-specific antigen (PSA) to a sandwich-type immunoassay format resulted in trapping of the photo-excited electrons by $\mathrm{Cu}^{2+}$ ions, leading to a decreased photocurrent. Under optimal conditions, the $\operatorname{Cdot} / g-\mathrm{C}_{3} \mathrm{~N}_{4}$ heterostructure showed a dynamic linear range of $0.02-100 \mathrm{ng} \mathrm{mL}^{-1}$ and calculated LOD of $5.0 \mathrm{pg} \mathrm{mL}{ }^{-1}$ (Fig. 15a and b). ${ }^{240} \mathrm{~A}$ Cdot-chitosan composite film modified GC working electrode was fabricated for electrochemical sensing of dopamine. Detailed analysis showed that the Cdot-chitosan film had substantial surface area and high conductivity. Reduction in resistance of the sensing platform due to Cdot integration resulted in improvement of the electrochemical performance. A linear response in the oxidation peak current was observed in the range between $0.1 \mu \mathrm{M}$ and $30.0 \mu \mathrm{M}$ with a 
calculated LOD of $11.2 \mathrm{nM}^{241}$ Cdots synthesized via thermal carbonization of ethylene glycol bis-(2-aminoethylether)- $N, N, N^{\prime}, N^{\prime}$ tetraacetic acid (EGTA) were used to prepare gold electrodes of DNA sensing devices. ${ }^{242} \mathrm{Li}$ et al. reported synthesis of ionic liquidfunctionalized graphitic Cdots, which exhibited good electron transfer properties. A Cdot functionalized GC electrode was then prepared and facile electron transport property was utilized for three electrode based electrochemical glucose sensing. ${ }^{243} \mathrm{CV}$ analysis showed a continuous decrease in the reduction peak with increasing glucose concentration. ${ }^{243} \mathrm{~N}$-rich Cdots were used to prepare a GC electrode for electrochemical reduction of TNT. ${ }^{244}$ The peak current corresponding to differential pulse voltammograms (DPVs) increased continuously with increasing TNT concentration. Amino functionalization allowed Cdots to accumulate TNT molecules through charge transfer between amine groups over Cdots and nitro aromatic groups of TNT, thus resulting in an efficient sensor response in this study (Fig. 15c). ${ }^{244}$ The presence of nanocrystalline $\mathrm{sp}^{2}$ hybridized domains was confirmed by the $0.21 \mathrm{~nm}$ plane in high-resolution TEM analysis. Such crystalline domains helped to reduce charge-transfer resistance $\left(R_{\mathrm{ct}}\right)$ in the as-prepared Cdot modified GC electrode. Lower $R_{\mathrm{ct}}$ suggested more electron-flux and improved electron shuttle between the substrate and electrolyte. Conducting Cdots, synthesized from citric acid monohydrate and ethylene diamine, were embedded inside the polymeric matrix of polypyrrole (PPy), which showed higher conductivity as compared to the pristine polymer. ${ }^{119}$ Conductivity of Cdot films was attributed to $\mathrm{sp}^{2} \mathrm{C}-\mathrm{C}$ bonds. By using the changes in $I-V$ characteristics of the composite material, a sensing device for picric acid detection was fabricated (Fig. 15d and e). During analyses, changes in the conductivity of the composite material were attributed to increasing picric acid concentration, which resulted in protonation of the polymer backbone, thus leading to a higher charge carrier density. ${ }^{119}$

Therefore, the sensitivity of electrochemical and conductometric sensors is dependent upon the charge transfer resistance (affecting the electron or hole transport ability) of the devices. It is believed that such an effect could be significantly improved by introducing structural alignment in the substrates (Cdots here), which will eventually enhance the charge carrier density in the sensing substrate, hence achieving better sensitivity along with selectivity.

\subsection{Photothermal energy conversion}

Photothermal conversion corresponds to generation of thermal energy or heat using the photoexcitation of materials, which is being actively proposed nowadays for theranostic applications especially in cancer treatment. So far, several organic dyes, semiconductor oxides, and metal nanoparticles have been used for efficient photothermal energy conversion and photothermal therapy (PTT). ${ }^{245}$ In this regard, use of plasmonic nanoparticles is noteworthy because of high absorption coefficients and efficient photothermal conversions within the range of biological transparency (650-950 nm). ${ }^{246-250}$ However, organic dyes exhibit weak thermal and photostability, whereas elimination of metal nanoparticles or semiconductor oxides is difficult following
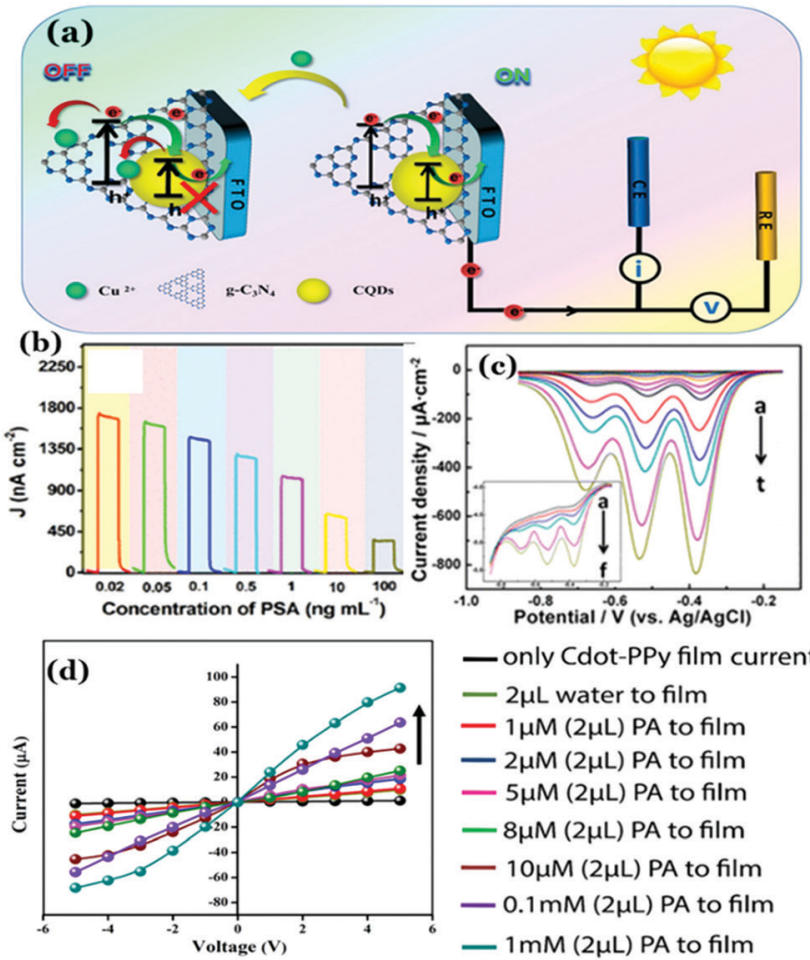

—only Cdot-PPy film current - $2 \mu \mathrm{L}$ water to film $-1 \mu \mathrm{M}(2 \mu \mathrm{L}) \mathrm{PA}$ to film

$-2 \mu \mathrm{M}(2 \mu \mathrm{L}) \mathrm{PA}$ to film - $5 \mu \mathrm{M}(2 \mu \mathrm{L}) \mathrm{PA}$ to film $-8 \mu \mathrm{M}(2 \mu \mathrm{L}) \mathrm{PA}$ to film

- $10 \mu \mathrm{M}(2 \mu \mathrm{L}) \mathrm{PA}$ to film - $0.1 \mathrm{mM}(2 \mu \mathrm{L})$ PA to film

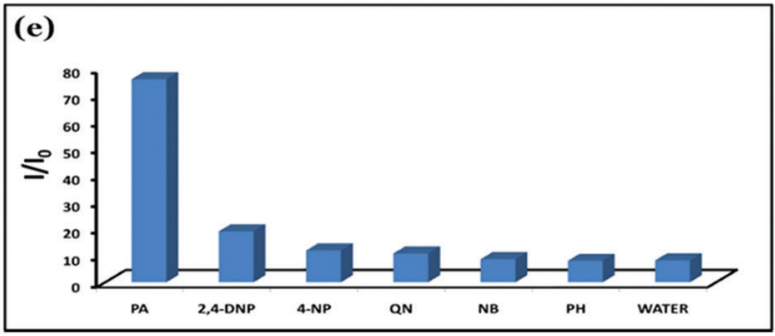

Fig. 15 (a) Mechanism of $\mathrm{Cu}^{2+}$-quenched photocurrent of Cdots $/ g-\mathrm{C}_{3} \mathrm{~N}_{4}$ heterostructures. (b) Photocurrent responses of the Cdots $/ g-\mathrm{C}_{3} \mathrm{~N}_{4}$ sensing platform towards prostate-specific antigen (PSA) with different concentrations. Reprinted with permission from ref. 240. Copyright 2017 American Chemical Society. (c) Differential pulse voltammograms (DPVs) responding to different concentrations of TNT at the CDs/GC electrode. Reprinted with permission from ref. 244. Copyright 2015 American Chemical Society. (d) I-V characteristics of the Cdot-PPy composite film in the presence of different concentrations of picric acid (PA) solutions. (e) The ratios of current flowing through the composite film following addition of $2.0 \mu \mathrm{L} 1.0 \mathrm{mM}$ aqueous solution of different analytes. In the figure $\mathrm{PA}=$ picric acid, 2,4-DNP = 2,4-dinitrophenol, 4-NP = 4-nitrophenol, NB = nitrobenzene, $\mathrm{PH}=$ phenol, $\mathrm{QN}=1$,4-benzoquinone, 4-MBA= 4-methoxybenzoic acid. The measurements were made at $+5 \mathrm{~V}$. Reprinted with permission from ref. 119. Copyright 2016 American Chemical Society.

treatment, thereby putting the human body at risk of heavy metal intoxication. ${ }^{245}$

On the other hand, because of their ease of synthesis, good biocompatibility and low toxicity Cdots are of keen research interest for PTT applications. Also, Cdots are found to have strong fluorescence, high absorption coefficient in the NIR region and high photothermal conversion efficiency. So far, graphitic crystalline Cdots have been utilized for efficient photothermal conversion and PTT. ${ }^{24,251-255}$ The strong absorbance of Cdots in the NIR region is attributed to various surface 
functional groups (resulting in lower energy transitions), Cdot assembly formation (due to strong electrostatic interactions among surface functional groups) and a large amount of pyrrolic-N (resulting in high electron density and extended delocalization). ${ }^{254}$ Additionally, graphitic arrangement inside the Cdot structure assists in smooth phonon transportation.

Wang and co-workers prepared red-emissive and graphitic Cdots from polythiophene phenylpropionic acid, which upon laser irradiation showed strong photothermal conversion. ${ }^{251}$ Cdot particles with a diameter of approximately $10 \mathrm{~nm}$ consisted of well-resolved crystal lattice consistent with the (100) plane of graphene. Wide-angle XRD showed a relatively broad peak around $23^{\circ}$ corresponding to the (002) facet. ${ }^{251}$ The conversion efficiency was found to be $\sim 38.5 \%$. This phenomenon was successfully applied as thermal theranostics against cancer (Fig. 16a-d). ${ }^{251}$ Recently, crystalline Cdots with a broad absorption range up to $1100 \mathrm{~nm}$ have been synthesized via hydrothermal treatment of 1,3,6-trinitropyrene and $\mathrm{Na}_{2} \mathrm{SO}_{3} \cdot{ }^{253}$ The photothermal conversion efficiency of the same was measured to be $\sim 52.3 \%$. Efficient photothermal heating of aqueous medium was attributed to their crystalline nature, which eventually assisted in good phonon transportation rather than phonon scattering loss. Thus, broad NIR absorption and significant photothermal conversion efficiency enabled the as-synthesized Cdots to be employed as a multifunctional platform for NIR imaging, photodynamic therapy (PDT) and PTT for cancer
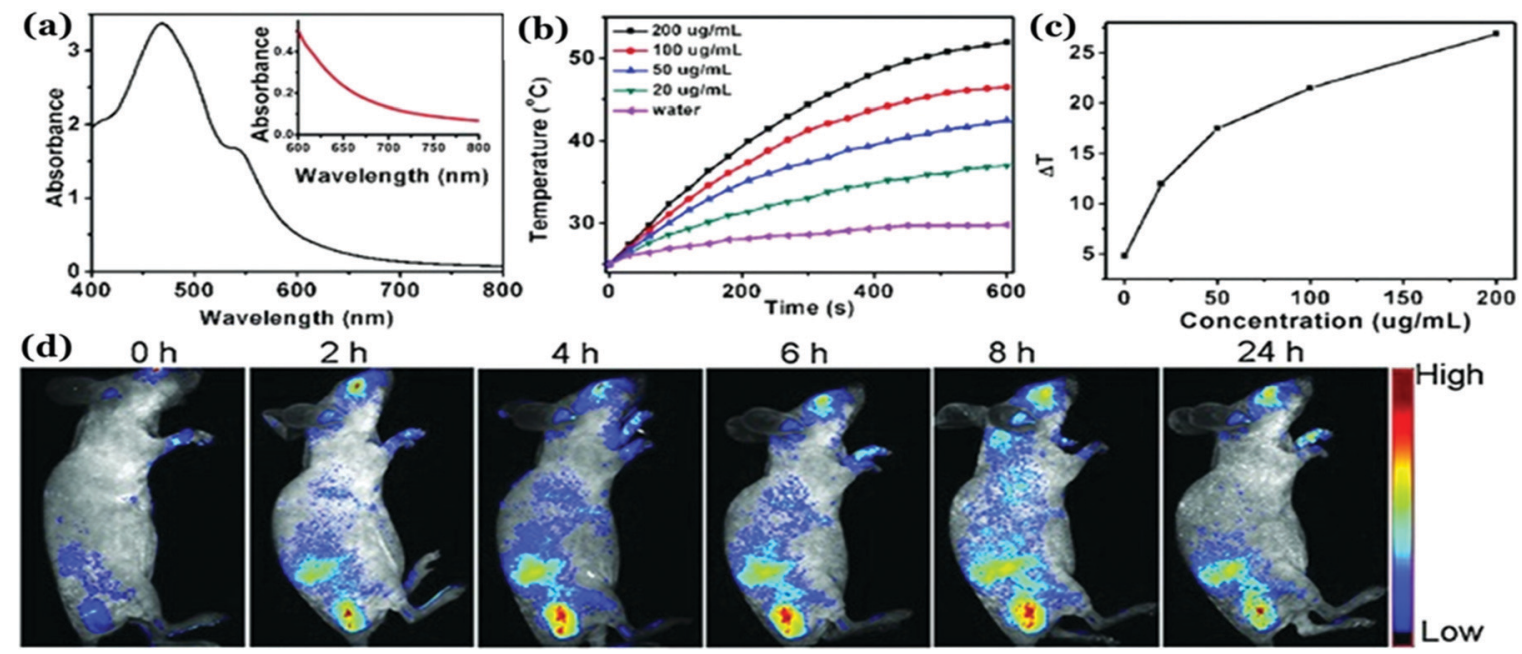

$2 \mathrm{~h}$

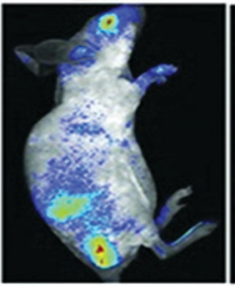

$4 \mathrm{~h}$

$6 \mathrm{~h}$
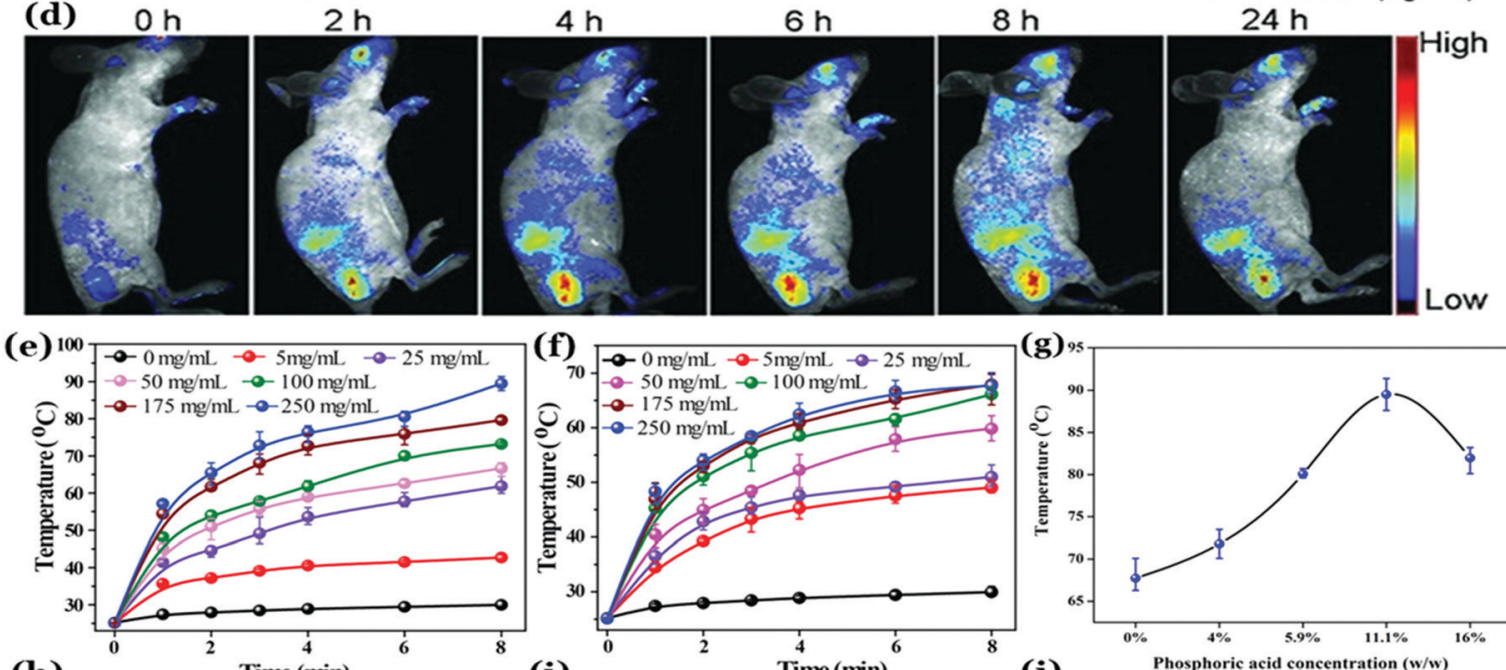

(h)
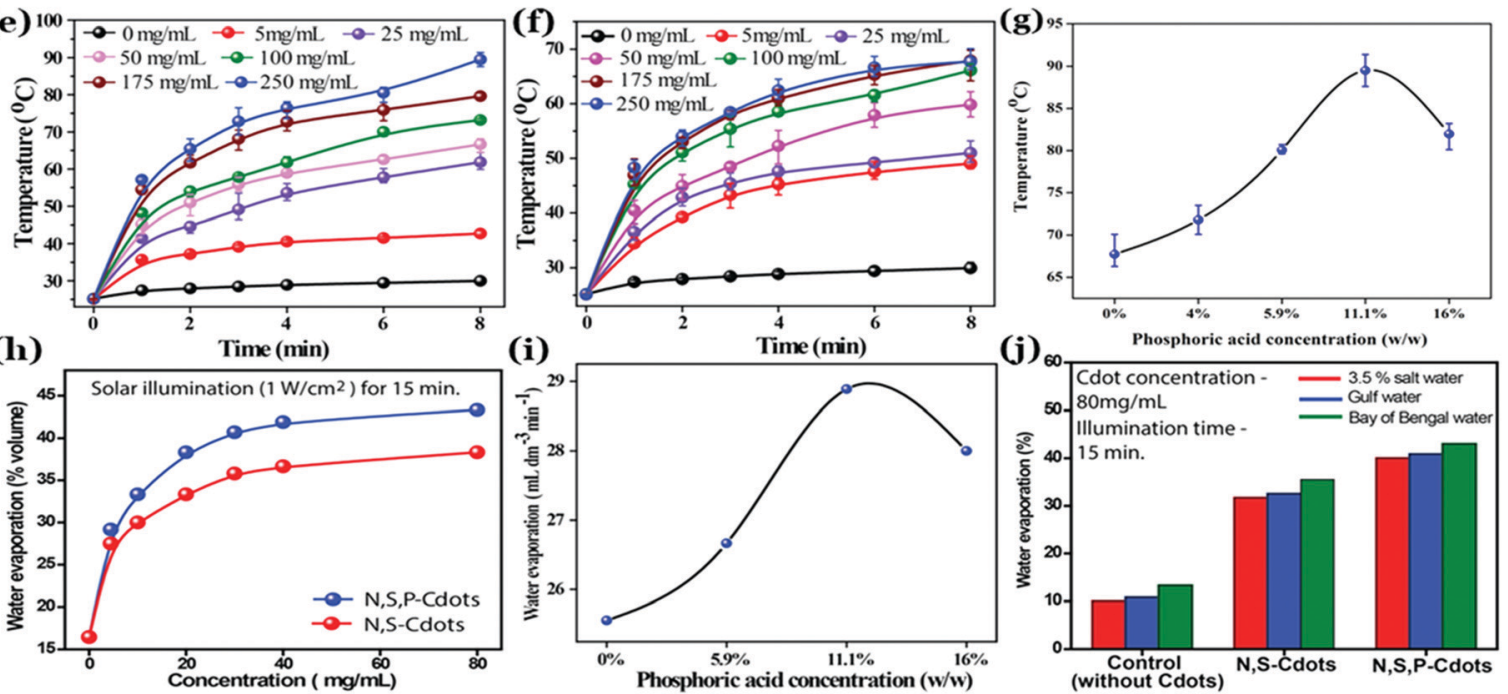

Fig. 16 (a) Absorption spectrum of Cdots and enlarged view of the same between 600 to $750 \mathrm{~nm}$ wavelength in the inset. (b) Temperature increase of Cdot aqueous dispersion due to the Cdot concentration increase following irradiation with a $671 \mathrm{~nm}$ laser source of $2 \mathrm{~W} \mathrm{~cm} \mathrm{~cm}^{-2}$ power density. (c) Plot of temperature difference for different Cdot concentrations over a period of $600 \mathrm{~s}$ laser irradiation. (d) In vivo red fluorescence images captured in real-time following intravenous injection of Cdots in mice at different time periods. (a-d) Reprinted with permission from ref. 251. Copyright 2015 John Wiley and Sons. Concentration dependent temperature increase by (e) non-graphitic highly crystalline Cdots (termed N,S,P-Cdots) and (f) mostly amorphous Cdots (termed N,S-Cdots; slightly graphitic) due to irradiation with a 2 W 808 nm laser source. (g) Photothermal study for N,S,P-Cdot-water dispersion showing maximum photothermal conversion due to maximum crystallinity in N,S,P-Cdots (i.e. $11.1 \%(\mathrm{w} / \mathrm{w})$ input concentration of $\mathrm{H}_{3} \mathrm{PO}_{4}$ ). (h) $\mathrm{Plots}$ corresponding to concentration dependent water evaporation by using the efficient photothermal conversion property of as-prepared crystalline Cdots. (i) Changes in the rate of water evaporation with increasing crystallinity in N,S,P-Cdots. (j) Extent of sea water desalinated using the Cdots in the presence of simulated solar radiation. (e-j) Reprinted with permission from ref. 127. Copyright 2018 Royal Society of Chemistry. 
treatment. ${ }^{253} \mathrm{Li}$ and co-workers reported $38.3 \%$ efficiency for nitrogen and oxygen co-doped graphitic Cdots. ${ }^{255}$ Though most of the reports suggest strong absorbance in the NIR region and the graphitic arrangements resulting in heat generation by Cdots, their limited crystalline nature in the structure is a major drawback, which restricts achievement of excellent photothermal conversion efficiency. It is important mentioning here that the photothermal energy conversion efficiency is not only dependent upon the photon absorption cross-section but also on the extent of crystalline order in the material. A recent study by our group revealed that high degree of crystallinity in the carbon nanostructure assists in efficient phonon transport and reduces phonon scattering losses in the nanostructure resulting in improved photothermal performance. ${ }^{127}$ Detailed studies revealed that doping of phosphorus inside the carbon nanostructure resulted in the generation of a high degree of well-defined polycrystalline domains, which finally assisted in efficient photothermal conversion. The temperature of the local environment could rise up to $89.5^{\circ} \mathrm{C}$ using $2 \mathrm{~W}, 808 \mathrm{~nm}$ IR laser irradiation for $8 \mathrm{~min}$. However, for non-crystalline Cdots the heat generation ability was found to be quite low. This phenomenon was finally implemented for solar thermal energy conversion and sea water desalination. Notably, the maximum solar photothermal evaporation efficiency was found to be $83.6 \%$ in the study for highly crystalline Cdots (Fig. 16e-j). ${ }^{127}$ Here, the efficiency value was significantly higher than the same for Cdots having finite graphitic arrangements. Halas and co-workers demonstrated efficient steam generation by using carbon nanoparticles in the presence of solar illumination. Thermodynamic analysis revealed that $80 \%$ of the absorbed solar radiation assisted in vaporization of water into steam whereas $20 \%$ of the absorbed radiation resulted in heating the fluid. This phenomenon was finally used for $\mathrm{H}_{2} \mathrm{O}$-ethanol distillation. ${ }^{256}$ The concept of bringing crystallinity in carbon nanostructures for efficient photothermal energy conversion is new but effective as it enhances thermal conductivity and reduces phonon scattering losses resulting in intense heat generation. Thus, higher order crystallinity in carbon nanostructures not only assists in efficient solar light assisted desalination and distillation, but also provides new direction in Cdot based PTT for cancer treatment.

\section{Conclusion and future perspectives}

In conclusion, we have highlighted the recent advancements made in Cdot research and their significant promise in energy related applications such as photovoltaics, optoelectronics, photocatalysis, electronic sensing, and building batteries and supercapacitors. Indeed, recent findings indicate that Cdots have the potential to reveal new interesting phenomena, even though several other aspects remain to be explored. The field of Cdots is expanding along with new ideas coming up persistently, demonstrating their facile synthesis methods, new structural characteristics and applicability. Despite extensive research on Cdot properties, the origin of their exceptional photoluminescence is still debatable. We summarize that Cdots can have three different types of emission mechanism. Apart from emission characteristics guided by the carbon core or surface defects, the luminescence of Cdots can be controlled by small organic molecular fluorophores as well, especially when citric acid and a few aromatic amines are used as the precursors. Incomplete carbonization of the precursor molecules often leads to the formation of amorphous carbon as the impurity, requiring efficient separation techniques to be employed following synthesis.

Unlike other allotropes, Cdots are largely amorphous in nature. They can have limited graphitic crystallinity depending upon precursors and synthesis techniques. This review article includes recent reports on structural engineering of Cdots especially for controlling crystallinity in Cdot structures. In fact, a few techniques have already been introduced such as heteroatom doping, self-assembly in order to generate or enhance long-range crystalline order in Cdots. Understanding the core structure and generation of crystalline domains in Cdots are crucial and may pave the way for a better understanding of their electronic transition and transportation properties. Of late, use of Cdots and corresponding composite materials for energy-based applications is worth mentioning. In fact, literature reports suggest that apart from being an emerging class of "nanolights", Cdots are also efficient energy harvesting materials.

By controlling the size, nature of surface functional groups and the extent of crystallinity the HOMO-LUMO energy gap in Cdots can be tuned for efficient utilization of the solar spectrum. Excellent electron donating and accepting abilities make Cdots appropriate for use in organic photovoltaic devices. In fact, Cdot based nanocomposites are regarded as efficient materials for preparing counter electrodes for dye-sensitized solar cells. Large specific surface area and surface-edge defects play pivotal roles for effective charge separation after photoexcitation and electron hole pair generation allowing efficient $\mathrm{CO}_{2}$ reduction and $\mathrm{H}_{2}$ evolution due to water splitting. Heteroatom doping further increases their catalytic activity. Although researchers have developed Cdot based optoelectronic devices, low QY in the solid state, limited structural arrangement and low emission in the longer wavelength region are the major drawbacks remaining to be addressed. Furthermore, better charge transport and/or phonon transport ability of Cdots allow them to be used in developing electronic sensors and energy storage devices and efficient photothermal conversion. In this context, generation of well-ordered crystalline domains in Cdot structures is going to be crucial, as the presence of such an ordered geometry provides conductive networks for efficient electronic transportation and charge separation. Therefore, we believe that the future of Cdots is bright both as luminescent and energy harvesting materials especially for the fabrication of low-cost, environmentally friendly and miniaturized devices. Thus, there is still opportunity to pursue further research on Cdots.

\section{Conflicts of interest}

The authors declare no competing financial interest. 


\section{Acknowledgements}

This work was supported by the Ministry of Electronics and Information Technology, Government of India (Grant 5(9)/ 2012-NANO, Vol. IV). MPS acknowledges the University Grants Commission for the UGC-BRS Research Start-Up-Grant; grant number F.30-377/2017(BSR). AC thanks the Department of Science and Technology (DST) for the J. C. Bose fellowship (JCB/2019/000039). AP thanks Arin Bhakat for assistance.

\section{References}

1 B. C. M. Martindale, G. A. M. Hutton, C. A. Caputo and E. Reisner, J. Am. Chem. Soc., 2015, 137, 6018-6025.

2 C. Hu, Y. Lin, J. W. Connell, H. Cheng, Y. Gogotsi, M. Titirici and L. Dai, Adv. Mater., 2019, 31, 1806128.

3 X. Zhang, P. K. Santra, L. Tian, M. B. Johansson, H. Rensmo and E. M. J. Johansson, ACS Nano, 2017, 11, 8478-8487.

4 T. Leijtens, T. Giovenzana, S. N. Habisreutinger, J. S. Tinkham, N. K. Noel, B. A. Kamino, G. Sadoughi, A. Sellinger and H. J. Snaith, ACS Appl. Mater. Interfaces, 2016, 8, 5981-5989.

5 N. Tetreault, E. Arsenault, L. Heiniger, N. Soheilnia, J. Brillet, T. Moehl, S. Zakeeruddin, G. A. Ozin and M. Gratzel, Nano Lett., 2011, 11, 4579-4584.

6 D. Wu, Z. Guo, X. Yin, Q. Pang, B. Tu, L. Zhang, Y. Wang and Q. Li, Adv. Mater., 2014, 26, 3258-3262.

7 F. Xu, S. Yang, X. Chen, Q. Liu, H. Li, H. Wang, B. Wei and D. Jiang, Chem. Sci., 2019, 10, 6001-6006.

8 S. D. Pritzl, F. Pschunder, F. Ehrat, S. Bhattacharyya, T. Lohmüller, M. A. Huergo and J. Feldmann, Nano Lett., 2019, 19, 3886-3891.

9 J. Tang, B. Kong, H. Wu, M. Xu, Y. Wang, Y. Wang, D. Zhao and G. Zheng, Adv. Mater., 2013, 25, 6569-6574.

10 M. P. Sk, U. Goswami, S. S. Ghosh and A. Chattopadhyay, J. Mater. Chem. B, 2015, 3, 5673-5677.

11 K. Jiang, S. Sun, L. Zhang, Y. Lu, A. Wu, C. Cai and H. Lin, Angew. Chem., Int. Ed., 2015, 54, 5360-5363.

12 S. K. Bhunia, A. Saha, A. Ranjan Maity, S. C. Ray and N. R. Jana, Sci. Rep., 2013, 3, 1473.

13 D. Chao, W. Lyu, Y. Liu, L. Zhou, Q. Zhang, R. Denga and H. Zhang, J. Mater. Chem. C, 2018, 6, 7527-7532.

14 A. Pal, K. Ahmad, D. Dutta and A. Chattopadhyay, ChemPhysChem, 2019, 20, 1018-1027.

15 G. Zuo, A. Xie, J. Li, T. Su, X. Pan and W. Dong, J. Phys. Chem. C, 2017, 121, 26558-26565.

16 M. P. Sk and A. Chattopadhyay, RSC Adv., 2014, 4, 31994-31999.

17 R. Liu, H. Huang, H. Li, Y. Liu, J. Zhong, Y. Li, S. Zhang and Z. Kang, ACS Catal., 2014, 4, 328-336.

18 M. P. Sk, C. K. Jana and A. Chattopadhyay, Chem. Commun., 2013, 49, 8235-8237.

19 S. Bhattacharyya, F. Ehrat, P. Urban, R. Teves, R. Wyrwich, M. Döblinger, J. Feldmann, A. S. Urban and J. K. Stolarczyk, Nat. Commun., 2017, 8, 1401.
20 K. A. S. Fernando, S. Sahu, Y. Liu, W. K. Lewis, E. A. Guliants, A. Jafariyan, P. Wang, C. E. Bunker and Y. Sun, ACS Appl. Mater. Interfaces, 2015, 7, 8363-8376.

21 R. Genc, M. O. Alas, E. Harputlu, S. Repp, N. Kremer, M. Castellano, S. G. Colak, K. Ocakoglu and E. Erdem, Sci. Rep., 2017, 7, 11222.

22 Y. Dang, S. Ren, G. Liu, J. Cai, Y. Zhang and J. Qiu, Nanomaterials, 2016, 6, 212.

23 J. Briscoe, A. Marinovic, M. Sevilla, S. Dunn and M. Titirici, Angew. Chem., Int. Ed., 2015, 54, 4463-4468.

24 H. Wang, J. Shen, Y. Li, Z. Wei, G. Cao, Z. Gai, K. Hong, P. Banerjee and S. Zhou, Biomater. Sci., 2014, 2, 915-923.

25 X. Xu, R. Ray, Y. Gu, H. J. Ploehn, L. Gearheart, K. Raker and W. A. Scrivens, J. Am. Chem. Soc., 2004, 126, 12736-12737.

26 S. N. Baker and G. A. Baker, Angew. Chem., Int. Ed., 2010, 49, 6726-6744.

27 S. Y. Lim, W. Shen and Z. Gao, Chem. Soc. Rev., 2015, 44, 362-381.

28 V. Georgakilas, J. A. Perman, J. Tucek and R. Zboril, Chem. Rev., 2015, 115, 4744-4822.

29 Y. Wang and A. Hu, J. Mater. Chem. C, 2014, 2, 6921-6939.

30 J. Zhang and S.-H. Yu, Mater. Today, 2016, 19, 382-393.

31 X. Shi, H. Meng, Y. Sun, L. Qu, Y. Lin, Z. Li and D. Du, Small, 2019, 15, 1901507.

32 C. Hu, M. Li, J. Qiu and Y. Sun, Chem. Soc. Rev., 2019, 48, 2315-2337.

33 J. Gao, M. Zhu, H. Huang, Y. Liu and Z. Kang, Inorg. Chem. Front., 2017, 4, 1963-1986.

34 R. Wang, K. Lu, Z. Tang and Y. Xu, J. Mater. Chem. A, 2017, 5, 3717-3734.

35 Y. Sun, B. Zhou, Y. Lin, W. Wang, K. A. S. Fernando, P. Pathak, Mo. J. Meziani, B. A. Harruff, X. Wang, H. Wang, P. G. Luo, H. Yang, M. E. Kose, B. Chen, L. M. Veca and S. Xie, J. Am. Chem. Soc., 2006, 128, 7756-7757.

36 H. Liu, T. Ye and C. Mao, Angew. Chem., Int. Ed., 2007, 46, 6473-6475.

37 X. Li, H. Wang, Y. Shimizu, A. Pyatenko, K. Kawaguchi and N. Koshizaki, Chem. Commun., 2011, 47, 932-934.

38 S. Hu, J. Liu, J. Yang, Y. Wang and S. Cao, J. Nanopart. Res., 2011, 13, 7247-7252.

39 N. Tarasenka, A. Stupak, N. Tarasenko, S. Chakrabarti and D. Mariotti, ChemPhysChem, 2017, 18, 1074-1083.

40 H. Li, X. He, Y. Liu, H. Yu, Z. Kang and S. Lee, Mater. Res. Bull., 2011, 46, 147-151.

41 Z. Qiao, Y. Wang, Y. Gao, H. Li, T. Dai, Y. Liu and Q. Huo, Chem. Commun., 2010, 46, 8812-8814.

42 J. Zhou, C. Booker, R. Li, X. Zhou, T. Sham, X. Sun and Z. Ding, J. Am. Chem. Soc., 2007, 129, 744-745.

43 H. Li, X. He, Z. Kang, H. Huang, Y. Liu, J. Liu, S. Lian, C. H. A. Tsang, X. Yang and S. Lee, Angew. Chem., Int. Ed., 2010, 49, 4430-4434.

44 Y. Long, C. Zhou, Z. Zhang, Z. Tian, L. Bao, Y. Lin and D. Pang, J. Mater. Chem., 2012, 22, 5917-5920.

45 J. Deng, Q. Lu, N. Mi, H. Li, Me. Liu, M. Xu, L. Tan, Q. Xie, Y. Zhang and S. Yao, Chem. - Eur. J., 2014, 20, 4993-4999. 
46 H. Zhu, X. Wang, Y. Li, Z. Wang, F. Yang and X. Yang, Chem. Commun., 2009, 5118-5120.

47 A. Jaiswal, S. S. Ghosh and A. Chattopadhyay, Chem. Commun., 2012, 48, 407-409.

48 K. Ahmad, A. Pal, U. N. Pan, A. Chattopadhyay and A. Paul, J. Mater. Chem. C, 2018, 6, 6691-6697.

49 D. Qu, M. Zheng, P. Du, Y. Zhou, L. Zhang, D. Li, H. Tan, Z. Zhao, Z. Xie and Z. Sun, Nanoscale, 2013, 5, 12272-12277.

50 H. Liu, Z. Li, Y. Sun, X. Geng, Y. Hu, H. Meng, J. Ge and L. Qu, Sci. Rep., 2018, 8, 1086.

51 J. Bian, C. Huang, L. Wang, T. Hung, W. A. Daoud and R. Zhang, ACS Appl. Mater. Interfaces, 2014, 6, 4883-4890.

52 P. Hsu and H. Chang, Chem. Commun., 2012, 48, 3984-3986.

53 M. K. Barman, B. Jana, S. Bhattacharyya and A. Patra, J. Phys. Chem. C, 2014, 118, 20034-20041.

54 M. P. Sk, A. Jaiswal, A. Paul, S. S. Ghosh and A. Chattopadhyay, Sci. Rep., 2012, 2, 383.

55 B. Chen, F. Li, S. Li, W. Weng, H. Guo, T. Guo, X. Zhang, Y. Chen, T. Huang, X. Hong, S. You, Y. Lin, K. Zeng and S. Chen, Nanoscale, 2013, 5, 1967-1971.

56 J. Wang, C.-F. Wang and S. Chen, Angew. Chem. Int. Ed., 2012, 51, 9297-9301.

57 H. Jiang, F. Chen, M. G. Lagally and F. S. Denes, Langmuir, 2010, 26(3), 1991-1995.

58 S. Y. Park, C. Y. Lee, H. An, H. Kim, Y. Lee, E. C. Park, H. Chun, H. Y. Yang, S. Choi, H. S. Kim, K. S. Kang, H. G. Park, J. Kim, Y. Choi, J. Lee and H. U. Lee, Nanoscale, 2017, 9, 9210-9217.

59 S. Y. Park, H. U. Lee, E. S. Park, S. C. Lee, J. Lee, S. W. Jeong, C. H. Kim, Y. Lee, Y. S. Huh and J. Lee, ACS Appl. Mater. Interfaces, 2014, 6, 3365-3370.

60 M. Han, S. Zhu, S. Lu, Y. Song, T. Feng, S. Tao, J. Liu and B. Yang, Nano Today, 2018, 19, 201-218.

61 X. Ren, F. Zhang, B. Guo, N. Gao and X. Zhang, Nanomaterials, 2019, 9, 495.

62 D. Tan, S. Zhou, Y. Shimotsuma, K. Miura and J. Qiu, Opt. Mater. Express, 2014, 4, 213-219.

63 B. Han, M. Yu, T. Pen, Y. Li, X. Hu, R. Xiang, X. Hou and G. He, New J. Chem., 2017, 41, 5267-5270.

64 D. Sun, R. Ban, P. Zhang, G. Wu, J. Zhang and J. Zhu, Carbon, 2013, 64, 424-434.

65 L. Tian, D. Ghosh, W. Chen, S. Pradhan, X. Chang and S. Chen, Chem. Mater., 2009, 21, 2803-2809.

66 J. Jiang, Y. He, S. Li and H. Cui, Chem. Commun., 2012, 48, 9634-9636.

67 D. Wang, L. Zhu, C. Mccleese, C. Burda, J. Chen and L. Dai, RSC Adv., 2016, 6, 41516-41521.

68 S. S. Monte-Filho, S. I. E. Andrade, M. B. Lima and M. C. U. Araujo, J. Pharm. Anal., 2019, 9, 209-216.

69 N. Papaioannou, A. Marinovic, N. Yoshizawa, A. E. Goode, M. Fay, A. Khlobystov, M. Titirici and A. Sapelkin, Sci. Rep., 2018, 8, 6559.

70 Y. Yang, J. Cui, M. Zheng, C. Hu, S. Tan, Y. Xiao, Q. Yang and Y. Liu, Chem. Commun., 2012, 48, 380-382.
71 S. Pei, J. Zhang, M. Gao, D. Wu, Y. Yang and R. Liu, J. Colloid Interface Sci., 2015, 439, 129-133.

72 L. Wang and H. S. Zhou, Anal. Chem., 2014, 86, 8902-8905.

73 J. Lia, L. Zhang, P. Li, Y. Zhang and C. Dong, Sens. Actuators, B, 2018, 258, 580-588.

74 S. Sahu, B. Behera, T. K. Maiti and S. Mohapatra, Chem. Commun., 2012, 48, 8835-8837.

75 A. Prasannan and T. Imae, Ind. Eng. Chem. Res., 2013, 52, 15673-15678.

76 C. Lai, Y. Hsiao, Y. Peng and P. Chou, J. Mater. Chem., 2012, 22, 14403-14409.

77 X. Liu, J. Pang, F. Xu and X. Zhang, Sci. Rep., 2016, 6, 31100 .

78 A. B. Siddique, A. K. Pramanick, S. Chatterjee and M. Ray, Sci. Rep., 2018, 8, 9770.

79 S. Zhu, Q. Meng, L. Wang, J. Zhang, Y. Song, H. Jin, K. Zhang, H. Sun, H. Wang and B. Yang, Angew. Chem., Int. Ed., 2013, 52, 3953-3957.

80 M. L. Liu, L. Yang, R. S. Li, B. B. Chen, H. Liu and C. Z. Huang, Green Chem., 2017, 19, 3611-3617.

81 K. Holá, M. Sudolská, S. Kalytchuk, D. Nachtigallová, A. L. Rogach, M. Otyepka and R. Zbořil, ACS Nano, 2017, 11, 12402-12410.

82 H. Ding, S. Yu, J. Wei and H. Xiong, ACS Nano, 2016, 10, 484-491.

83 W. Kwon and S. Rhee, Chem. Commun., 2012, 48, 5256-5258.

84 S. Hu, K. Niu, J. Sun, J. Yang, N. Zhao and X. Du, J. Mater. Chem., 2009, 19, 484-488.

85 M. Righetto, A. Privitera, I. Fortunati, D. Mosconi, M. Zerbetto, M. L. Curri, M. Corricelli, A. Moretto, S. Agnoli, L. Franco, R. Bozio and C. Ferrante, J. Phys. Chem. Lett., 2017, 8, 2236-2242.

86 Y. Wang, Q. Zhuang and Y. Ni, Chem. - Eur. J., 2015, 21, 13004-13011.

87 K. Hola, A. B. Bourlinos, O. Kozak, K. Berka, K. M. Siskova, M. Havrdova, J. Tucek, K. Safarova, M. Otyepka, E. P. Giannelis and R. Zboril, Carbon, 2014, 70, 279-286.

88 W. Kwon, G. Lee, S. Do, T. Joo and S. Rhee, Small, 2014, 10, 506-513.

89 Y. Park, J. Yoo, B. Lim, W. Kwon and S.-W. Rhee, J. Mater. Chem. A, 2016, 4, 11582-11603.

90 J. T. Margraf, V. Strauss, D. M. Guldi and T. Clark, J. Phys. Chem. B, 2015, 119, 7258-7265.

91 X. Gong, Q. Zhang, Y. Gao, S. Shuang, M. M. F. Choi and C. Dong, ACS Appl. Mater. Interfaces, 2016, 8, 11288-11297.

92 V. Borse, M. Thakur, S. Sengupta and R. Srivastava, Sens. Actuators, B, 2017, 248, 481-492.

93 A. B. Bourlinos, G. Trivizas, M. A. Karakassides, M. Baikousi, A. Kouloumpis, D. Gournis, A. Bakandritsos, K. Hola, O. Kozak, R. Zboril, I. Papagiannouli, P. Aloukos and S. Couris, Carbon, 2015, 83, 173-179.

94 L. Lin and S. Zhang, Chem. Commun., 2012, 48, 10177-10179. 95 J. Schneider, C. J. Reckmeier, Y. Xiong, M. v. Seckendorff, A. S. Susha, P. Kasák and A. L. Rogach, J. Phys. Chem. C, 2017, 121, 2014-2022. 
96 Y. Zhang, X. Liu, Y. Fan, X. Guo, L. Zhou, Y. Lv and J. Lin, Nanoscale, 2016, 8, 15281-15287.

97 Y. Xie, J. Zheng, Y. Wang, J. Wang, Y. Yang, X. Liu and Y. Chen, Nanotechnology, 2019, 30, 085406.

98 F. Yuan, Z. Wang, X. Li, Y. Li, Z. Tan, L. Fan and S. Yang, Adv. Mater., 2017, 29, 1604436.

99 L. Bao, C. Liu, Z. Zhang and D. Pang, Adv. Mater., 2015, 27, 1663-1667.

100 S. Hu, A. Trinchi, P. Atkin and I. Cole, Angew. Chem., Int. Ed., 2015, 54, 2970-2974.

101 X. Jia, J. Li and E. Wang, Nanoscale, 2012, 4, 5572-5575.

102 C. Wang, X. Wu, X. Li, W. Wang, L. Wang, M. Gu and Q. Li, J. Mater. Chem., 2012, 22, 15522-15525.

103 Z. Lin, W. Xue, H. Chen and J. Lin, Chem. Commun., 2012, 48, 1051-1053.

104 L. Zhao, F. Di, D. Wang, L. Guo, Y. Yang, B. Wan and H. Zhang, Nanoscale, 2013, 5, 2655-2658.

105 Y. Xu, M. Wu, X. Feng, X. Yin, X. He and Y. Zhang, Chem. Eur. J., 2013, 19, 6282-6288.

106 G. Eda, Y. Lin, C. Mattevi, H. Yamaguchi, H. Chen, I. Chen, C. Chen and M. Chhowalla, Adv. Mater., 2010, 22, 505-509.

107 M. A. Sk, A. Ananthanarayanan, L. Huang, K. H. Lim and P. Chen, J. Mater. Chem. C, 2014, 2, 6954-6960.

108 M. X. Gao, C. F. Liu, Z. L. Wu, Q. L. Zeng, X. X. Yang, W. B. Wu, Y. F. Lia and C. Z. Huang, Chem. Commun., 2013, 49, 8015-8017.

109 L. Guo, J. Ge, W. Liu, G. Niu, Q. Jia, H. Wang and P. Wang, Nanoscale, 2016, 8, 729-734.

110 M. J. Krysmann, A. Kelarakis, P. Dallas and E. P. Giannelis, J. Am. Chem. Soc., 2012, 134, 747-750.

111 Y. Song, S. Zhu, S. Zhang, Y. Fu, L. Wang, X. Zhao and B. Yang, J. Mater. Chem. C, 2015, 3, 5976-5984.

112 T. Zhang, J. Zhu, Y. Zhai, H. Wang, X. Bai, B. Dong, H. Wang and H. Song, Nanoscale, 2017, 9, 13042-13051.

113 W. Wang, B. Wang, H. Embrechts, C. Damm, A. Cadranel, V. Strauss, M. Distaso, V. Hinterberger, D. M. Guldi and W. Peukert, RSC Adv., 2017, 7, 24771-24780.

114 X. Wang, L. Cao, F. Lu, M. J. Meziani, H. Li, G. Qi, B. Zhou, B. A. Harruff, F. Kermarrec and Y. Sun, Chem. Commun., 2009, 3774-3776.

115 H. Zhang, H. Huang, H. Ming, H. Li, L. Zhang, Y. Liu and Z. Kang, J. Mater. Chem., 2012, 22, 10501-10506.

116 P. Yu, X. Wen, Y. Toh, Y. Lee, K. Huang, S. Huang, S. Shrestha, G. Conibeer and J. Tang, J. Mater. Chem. C, 2014, 2, 2894-2901.

117 X. Zhang, H. Ming, R. Liu, X. Han, Z. Kang, Y. Liu and Y. Zhang, Mater. Res. Bull., 2013, 48, 790-794.

118 L. Bhattacharjee, R. Manoharan, K. Mohanta and R. R. Bhattacharjee, J. Mater. Chem. A, 2015, 3, 1580-1586.

119 A. Pal, M. P. Sk and A. Chattopadhyay, ACS Appl. Mater. Interfaces, 2016, 8, 5758-5762.

120 H. Wu, Y. Chen, X. Dai, P. Li, J. F. Stoddart and Y. Liu, J. Am. Chem. Soc., 2019, 141, 6583-6591.

121 V. Strauss, J. T. Margraf, C. Dolle, B. Butz, T. J. Nacken, J. Walter, W. Bauer, W. Peukert, E. Spiecker, T. Clark and D. M. Guldi, J. Am. Chem. Soc., 2014, 136, 17308-17316.
122 A. B. Siddique, V. P. Singh, S. Chatterjee, A. K. Pramanik and M. Ray, Mater. Today: Proc., 2018, 5, 10077-10083.

123 W. Wang, C. Damm, J. Walter, T. J. Nacken and W. Peukert, Phys. Chem. Chem. Phys., 2016, 18, 466-475.

124 B. J. Moon, Y. Oh, D. H. Shin, S. J. Kim, S. H. Lee, T. Kim, M. Park and S. Bae, Chem. Mater., 2016, 28, 1481-1488.

125 B. Shi, D. Nachtigallova, A. J. A. Aquino, F. B. C. Machado and H. Lischka, Phys. Chem. Chem. Phys., 2019, 21, 9077-9088.

126 X. Sun, C. Brückner and Y. Lei, Nanoscale, 2015, 7, 17278-17282.

127 A. Pal, G. Natu, K. Ahmad and A. Chattopadhyay, J. Mater. Chem. A, 2018, 6, 4111-4118.

128 A. Pal, A. Bhakat and A. Chattopadhyay, J. Phys. Chem. C, 2019, 123, 19421-19428.

129 A. J. Nozik, M. C. Beard, J. M. Luther, M. Law, R. J. Ellingson and J. C. Johnson, Chem. Rev., 2010, 110, 6873-6890.

130 Y. Meng, Y. Zhang, W. Sun, M. Wang, B. He, H. Chen and Q. Tang, Electrochim. Acta, 2017, 257, 259-266.

131 P. Gao, K. Ding, Y. Wang, K. Ruan, S. Diao, Q. Zhang, B. Sun and J. Jie, J. Phys. Chem. C, 2014, 118, 5164-5171.

132 V. Gupta, N. Chaudhary, R. Srivastava, G. D. Sharma, R. Bhardwaj and S. Chand, J. Am. Chem. Soc., 2011, 133, 9960-9963.

133 Z. Ding, Z. Hao, B. Meng, Z. Xie, J. Liu and L. Dai, Nano Energy, 2015, 15, 186-192.

134 L. Zhang, Z. C. Ding, T. Tong and J. Liu, Nanoscale, 2017, 9, 3524-3529.

135 C. X. Guo, H. B. Yang, Z. M. Sheng, Z. S. Lu, Q. L. Song and C. M. Li, Angew. Chem., Int. Ed., 2010, 49, 3014-3017.

136 C. Lee, K. Lai, C. Lin, C. Li, K. Ho, C. Wu, S. Lau and J. He, Nano Energy, 2017, 36, 260-267.

137 Y. Li, Y. Hu, Y. Zhao, G. Shi, L. Deng, Y. Hou and L. Qu, Adv. Mater., 2011, 23, 776-780.

138 H. Choi1, S. Ko, Y. Choi, P. Joo, T. Kim, B. R. Lee, J. Jung, H. J. Choi, M. Cha, J. Jeong, I. Hwang, M. H. Song, B. Kim and J. Y. Kim, Nat. Photonics, 2013, 7, 732-738.

139 L. Yan, Y. Yang, C. Ma, X. Liu, H. Wang and B. Xu, Carbon, 2016, 109, 598-607.

140 Y. Wang, L. Yan, G. Ji, C. Wang, H. Gu, Q. Luo, Q. Chen, L. Chen, Y. Yang, C. Ma and X. Liu, ACS Appl. Mater. Interfaces, 2019, 11, 2243-2253.

141 X. Zhang, C. Liu, Z. Li, J. Guo, L. Shen, W. Guo, L. Zhang, S. Ruan and Y. Long, Chem. Eng. J., 2017, 315, 621-629.

142 M. Semeniuk, Z. Yi, V. Poursorkhabi, J. Tjong, S. Jaffer, Z. Lu and M. Sain, ACS Nano, 2019, 13(6), 6224-6255.

143 K. Lee, S. Cho, M. Kim, J. Kim, J. Ryu, K. Shin and J. Jang, J. Mater. Chem. A, 2015, 3, 19018-19026.

144 W. Zhu, Y. Zhao, J. Duan, Y. Duan, Q. Tang and B. He, Chem. Commun., 2017, 53, 9894-9897.

145 A. Kojima, K. Teshima, Y. Shirai and T. Miyasaka, J. Am. Chem. Soc., 2009, 131, 6050-6051.

146 J. Im, C. Lee, J. Lee, S. Park and N. Park, Nanoscale, 2011, 3, 4088-4093.

147 M. M. Lee, J. Teuscher, T. Miyasaka, T. N. Murakami and H. J. Snaith, Science, 2012, 338, 643-647. 
148 M. Liu, M. B. Johnston and H. J. Snaith, Nature, 2013, 501, 395-398.

149 J. M. Ball, M. M. Lee, A. Hey and H. J. Snaith, Energy Environ. Sci., 2013, 6, 1739-1743.

150 J. Tong, Z. Song, D. H. Kim, X. Chen, C. Chen, A. F. Palmstrom, P. F. Ndione, M. O. Reese, S. P. Dunfield, O. G. Reid, J. Liu, F. Zhang, S. P. Harvey, Z. Li, S. T. Christensen, G. Teeter, D. Zhao, M. M. Al-Jassim, M. F. A. M. van Hest, M. C. Beard, S. E. Shaheen, J. J. Berry, Y. Yan and K. Zhu, Science, 2019, 364, 475-479.

151 Y. Wang, J. Zhang, S. Chen, H. Zhang, L. Li and Z. Fu, J. Mater. Sci., 2018, 53, 9180-9190.

152 H. Li, W. Shi, W. Huang, E. Yao, J. Han, Z. Chen, S. Liu, Y. Shen, M. Wang and Y. Yang, Nano Lett., 2017, 17, 2328-2335.

153 C. Xie, B. Nie, L. Zeng, F. Liang, M. Wang, L. Luo, M. Feng, Y. Yu, C. Wu, Y. Wu and S. Yu, ACS Nano, 2014, 8(4), 4015-4022.

154 Z. Jiang, Y. Liu, T. Jing, B. Huang, X. Zhang, X. Qin, Y. Dai and M. Whangbo, J. Phys. Chem. C, 2016, 120, 2058-2063.

155 J. Liu, Y. Zhang, L. Lu, G. Wu and W. Chen, Chem. Commun., 2012, 48, 8826-8828.

156 Y. Yang, T. Zhang, L. Le, X. Ruan, P. Fang, C. Pan, R. Xiong, J. Shi and J. Wei, Sci. Rep., 2014, 4, 7045.

157 I. Tsuji, H. Kato and A. Kudo, Angew. Chem., Int. Ed., 2005, 44, 3565-3568.

158 C. A. Jaramillo-Páeza, J. A. Navíoa, M. C. Hidalgo and M. Macías, Catal. Today, 2018, 313, 12-19.

159 J. Liu, Y. Liu, N. Liu, Y. Han, X. Zhang, H. Huang, Y. Lifshitz, S. Lee, J. Zhong and Z. Kang, Science, 2015, 347, 970-974.

160 X. Wu, J. Zhao, S. Guo, L. Wang, W. Shi, H. Huang, Y. Liu and Z. Kang, Nanoscale, 2016, 8, 17314-17321.

161 J. Ke, X. Li, Q. Zhao, B. Liu, S. Liu and S. Wang, J. Colloid Interface Sci., 2017, 496, 425-433.

162 H. Yu, H. Zhang, H. Huang, Y. Liu, H. Li, H. Ming and Z. Kang, New J. Chem., 2012, 36, 1031-1035.

163 H. Zhang, H. Ming, S. Lian, H. Huang, H. Li, L. Zhang, Y. Liu, Z. Kang and S. Lee, Dalton Trans., 2011, 40, 10822-10825.

164 N. Chai, H. Wang, C. Hu, Q. Wang and H. Zhang, J. Mater. Chem. A, 2015, 3, 16613-16620.

165 J. Liu, H. Zhang, D. Tang, X. Zhang, L. Yan, Y. Han, H. Huang, Y. Liu and Z. Kang, ChemCatChem, 2014, 6, 2634-2641.

166 Y. Sun, Z. Zhang, A. Xie, C. Xiao, S. Li, F. Huang and Y. Shen, Nanoscale, 2015, 7, 13974-13980.

167 P. Zhang, T. Wang, X. Chang, L. Zhang and J. Gong, Angew. Chem., Int. Ed., 2016, 55, 5851-5855.

168 W. Li, Y. Liu, M. Wu, X. Feng, S. A. T. Redfern, Y. Shang, X. Yong, T. Feng, K. Wu, Z. Liu, B. Li, Z. Chen, J. S. Tse, S. Lu and B. Yang, Adv. Mater., 2018, 30, 1800676.

169 D. Tang, J. Liu, X. Wu, R. Liu, X. Han, Y. Han, H. Huang, Y. Liu and Z. Kang, ACS Appl. Mater. Interfaces, 2014, 6, 7918-7925.

170 D. Chen, S. Dai, X. Su, Y. Xin, S. Zou, X. Wang, Z. Kang and M. Shen, Chem. Commun., 2015, 51, 15340-15343.
171 S. Sahu, Y. Liu, P. Wang, C. E. Bunker, K. A. S. Fernando, W. K. Lewis, E. A. Guliants, F. Yang, J. Wang and Y. Sun, Langmuir, 2014, 30, 8631-8636.

172 L. Cao, S. Sahu, P. Anilkumar, C. E. Bunker, J. Xu, K. A. S. Fernando, P. Wang, E. A. Guliants, K. N. Tackett and Y. Sun, J. Am. Chem. Soc., 2011, 133, 4754-4757.

173 H. Li, R. Liu, S. Lian, Y. Liu, H. Huang and Z. Kang, Nanoscale, 2013, 5, 3289-3297.

174 H. Li, R. Liu, W. Kong, J. Liu, Y. Liu, L. Zhou, X. Zhang, S. Lee and Z. Kang, Nanoscale, 2014, 6, 867-873.

175 S. Hu, R. Tian, L. Wu, Q. Zhao, J. Yang, J. Liu and S. Cao, Chem. - Asian J., 2013, 8, 1035-1041.

176 S. Cailotto, R. Mazzaro, F. Enrichi, A. Vomiero, M. Selva, E. Cattaruzza, D. Cristofori, E. Amadio and A. Perosa, ACS Appl. Mater. Interfaces, 2018, 10, 40560-40567.

177 V. Ramar, S. Moothattu and K. Balasubramanian, Sol. Energy, 2018, 169, 120-127.

178 X. Guo, C. Wang, Z. Yu, L. Chen and S. Chen, Chem. Commun., 2012, 48, 2692-2694.

179 Q. Chen, C. Wang and S. Chen, J. Mater. Sci., 2013, 48, 2352-2357.

180 M. Sun, S. Qu, Z. Hao, W. Ji, P. Jing, H. Zhang, L. Zhang, J. Zhao and D. Shen, Nanoscale, 2014, 6, 13076-13081.

181 S. Do, W. Kwon and S. Rhee, J. Mater. Chem. C, 2014, 2, 4221-4226.

182 H. Tetsuka, A. Nagoya and R. Asahi, J. Mater. Chem. C, 2015, 3, 3536-3541.

183 C. Shen, J. Wang, Y. Cao and Y. Lu, J. Mater. Chem. C, 2015, 3, 6668-6675.

184 Z. Fan, Y. Li, X. Li, L. Fan, S. Zhou, D. Fang and S. Yang, Carbon, 2014, 70, 149-156.

185 F. Wang, Y. Chen, C. Liu and D. Ma, Chem. Commun., 2011, 47, 3502-3504.

186 F. Yuan, T. Yuan, L. Sui, Z. Wang, Z. Xi, Y. Li, X. Li, L. Fan, Z. Tan, A. Chen, M. Jin and S. Yang, Nat. Commun., 2018, 9, 2249.

187 J. Xu, Y. Miao, J. Zheng, H. Wang, Y. Yang and X. Liu, Nanoscale, 2018, 10, 11211-11221.

188 F. Béguin, V. Presser, A. Balducci and E. Frackowiak, Adv. Mater., 2014, 26, 2219-2251.

189 P. Simon, Y. Gogotsi and B. Dunn, Science, 2014, 343, 1210-1211.

190 X. Wang, X. Lu, B. Liu, D. Chen, Y. Tong and G. Shen, Adv. Mater., 2014, 26, 4763-4782.

191 J. Liu, M. Zheng, X. Shi, H. Zeng and H. Xia, Adv. Funct. Mater., 2016, 26, 919-930.

192 M. Jing, C. Wang, H. Hou, Z. Wu, Y. Zhu, Y. Yang, X. Jia, Y. Zhang and X. Ji, J. Power Sources, 2015, 298, 241-248.

193 B. Y. Guan, A. Kushima, L. Yu, S. Li, J. Li and X. W. Lou, Adv. Mater., 2017, 29, 1605902.

194 H. Yin, S. Zhao, Ji. Wan, H. Tang, L. Chang, L. He, H. Zhao, Y. Gao and Z. Tang, Adv. Mater., 2013, 25, 6270-6276.

195 S. Zhang, L. Sui, H. Dong, W. He, L. Dong and L. Yu, ACS Appl. Mater. Interfaces, 2018, 10, 12983-12991.

196 L. Li, Z. A. Hu, N. An, Y. Y. Yang, Z. M. Li and H. Y. Wu, J. Phys. Chem. C, 2014, 118, 22865-22872. 
197 Z. Zhao, S. Hao, P. Hao, Y. Sang, A. Manivannan, N. Wu and H. Liu, J. Mater. Chem. A, 2015, 3, 15049-15056.

198 Z. Song, D. Zhu, L. Li, T. Chen, H. Duan, Z. Wang, Y. Lv, W. Xiong, M. Liu and L. Gan, J. Mater. Chem. A, 2019, 7, 1177-1186.

199 H. Peng, B. Yao, X. Wei, T. Liu, T. Kou, P. Xiao, Y. Zhang and Y. Li, Adv. Energy Mater., 2019, 1803665.

200 Y. Tan, Y. Liu, Z. Tang, Z. Wang, L. Kong, L. Kang, Z. Liu and F. Ran, Sci. Rep., 2018, 8, 2915.

201 Z. Peng, Y. Hu, J. Wang, S. Liu, C. Li, Q. Jiang, J. Lu, X. Zeng, P. Peng and F. Li, Adv. Energy Mater., 2019, 1802928.

202 V. Palomares, P. Serras, I. Villaluenga, K. B. Hueso, J. Carretero-Gonzalez and T. Rojo, Energy Environ. Sci., 2012, 5, 5884-5901.

203 M. Jing, J. Wang, H. Hou, Y. Yang, Y. Zhang, C. Pan, J. Chen, Y. Zhu and X. Ji, J. Mater. Chem. A, 2015, 3, 16824-16830.

204 J. Wang, X. Zhang, W. He, Y. Yue, Y. Wang and C. Zhang, RSC Adv., 2017, 7, 2658-2666.

205 M. S. Balogun, Y. Luo, F. Lyu, F. Wang, H. Yang, H. Li, C. Liang, M. Huang, Y. Huang and Y. Tong, ACS Appl. Mater. Interfaces, 2016, 8, 9733-9744.

206 H. Hou, C. E. Banks, M. Jing, Y. Zhang and X. Ji, Adv. Mater., 2015, 27, 7861-7866.

207 Y. Yang, X. Ji, M. Jing, H. Hou, Y. Zhu, L. Fang, X. Yang, Q. Chena and C. E. Banks, J. Mater. Chem. A, 2015, 3, 5648-5655.

208 H. J. Becker, US Pat., 2800616 A, 1957.

209 Y. Zhang, B. Lin, J. Wang, J. Tian, Y. Sun, X. Zhang and H. Yang, J. Mater. Chem. A, 2016, 4, 10282-10293.

$210 \mathrm{X} . \mathrm{Xu}, \mathrm{S}$. Li, H. Zhang, Y. Shen, S. M. Zakeeruddin, M. Graetzel, Yi. Cheng and M. Wang, ACS Nano, 2015, 9(2), 1782-1787.

211 X. Du, C. Wang, M. Chen, Y. Jiao and J. Wang, J. Phys. Chem. C, 2009, 113, 2643-2646.

212 R. Liu and S. B. Lee, J. Am. Chem. Soc., 2008, 130, 2942-2943.

213 D. Sheberla, J. C. Bachman, J. S. Elias, C. Sun, Y. ShaoHorn and M. Dinca, Nat. Mater., 2017, 16, 220-225.

214 Y. Zhu, X. Ji, C. Pan, Q. Sun, W. Song, L. Fang, Q. Chen and C. E. Banks, Energy Environ. Sci., 2013, 6, 3665-3675.

215 Y. Zhu, Z. Wu, M. Jing, H. Hou, Y. Yang, Y. Zhang, X. Yang, W. Song, X. Jia and X. Ji, J. Mater. Chem. A, 2015, 3, 866-877.

216 B. Unnikrishnan, C. Wu, I. P. Chen, H. Chang, C. Lin and C. Huang, ACS Sustainable Chem. Eng., 2016, 4, 3008-3016.

217 Y. Wei, X. Zhang, X. Wu, D. Tang, K. Caia and Q. Zhang, RSC Adv., 2016, 6, 39317-39322.

218 J. Xu, Y. Xue, J. Cao, G. Wang, Y. Li, W. Wang and Z. Chen, RSC Adv., 2016, 6, 5541-5546.

219 B. De, J. Balamurugan, N. H. Kim and J. H. Lee, ACS Appl. Mater. Interfaces, 2017, 9, 2459-2468.

220 V. B. Kumar, A. Borenstein, B. Markovsky, D. Aurbach, A. Gedanken, M. Talianker and Z. Porat, J. Phys. Chem. C, 2016, 120, 13406-13413.
221 L. Lv, Y. Fan, Q. Chen, Y. Zhao, Y. Hu, Z. Zhang, N. Chen and L. Qu, Nanotechnology, 2014, 25, 235401.

222 B. Devadas and T. Imae, ACS Sustainable Chem. Eng., 2018, 6, 127-134.

223 X. Zhang, J. Wang, J. Liu, J. Wu, H. Chen and H. Bi, Carbon, 2017, 115, 134-146.

224 J. Wei, C. Ding, P. Zhang, H. Ding, X. Niu, Y. Ma, C. Li, Y. Wang and H. Xiong, Adv. Mater., 2019, 31, 1806197.

225 Q. Li, H. Cheng, X. Wu, C. Wang, G. Wu and S. Chen, J. Mater. Chem. A, 2018, 6, 14112-14119.

226 G. Chen, S. Wu, L. Hui, Y. Zhao, J. Ye, Z. Tan, W. Zeng, Z. Tao, L. Yang and Y. Zhu, Sci. Rep., 2016, 6, 19028.

227 F. Arshad, A. Pal, M. A. Rahman, M. Ali, J. A. Khan and M. P. Sk, New J. Chem., 2018, 42, 19837-19843.

228 W. Wei, C. Xu, J. Ren, B. Xu and X. Qu, Chem. Commun., 2012, 48, 1284-1286.

229 J. He, H. Zhang, J. Zou, Y. Liu, J. Zhuang, Y. Xiao and B. Lei, Biosens. Bioelectron., 2016, 79, 531-535.

230 M. L. Liu, B. B. Chen, C. M. Li and C. Z. Huang, Green Chem., 2019, 21, 449-471.

231 K. Qu, J. Wang, J. Ren and X. Qu, Chem. - Eur. J., 2013, 19, 7243-7249.

232 W. Wang, J. Xia, J. Feng, M. He, M. Chen and J. Wang, J. Mater. Chem. B, 2016, 4, 7130-7137.

233 B. B. Chen, Z. X. Liu, W. C. Deng, L. Zhan, M. Li Liu and C. Z. Huang, Green Chem., 2016, 18, 5127-5132.

234 N. Dhenadhayalan and K. Lin, Sci. Rep., 2015, 5, 10012.

235 S. Bhattacharya, R. Sarkar, B. Chakraborty, A. Porgador and R. Jelinek, ACS Sens., 2017, 2, 1215-1224.

236 F. Arshad and M. P. Sk, New J. Chem., 2019, 43, 13240-13248.

237 Y. Wang, C. Zhang, X. Chen, B. Yang, L. Yang, C. Jiang and Z. Zhang, Nanoscale, 2016, 8, 5977-5984.

238 J. Chen, J. Wei, P. Zhang, X. Niu, W. Zhao, Z. Zhu, H. Ding and H. Xiong, ACS Appl. Mater. Interfaces, 2017, 9, 18429-18433.

239 Y. Wang, Z. Wang, Y. Rui and M. Li, Biosens. Bioelectron., 2015, 64, 57-62.

240 S. Lv, Y. Li, K. Zhang, Z. Lin and D. Tang, ACS Appl. Mater. Interfaces, 2017, 9, 38336-38343.

241 Q. Huang, S. Hu, H. Zhang, J. Chen, Y. He, F. Li, W. Weng, J. Ni, X. Bao and Y. Lin, Analyst, 2013, 138, 5417-5423.

242 T. García-Mendiola, I. Bravo, J. M. López-Moreno, F. Pariente, R. Wannemacher, K. Weber, J. Popp and E. Lorenzo, Sens. Actuators, B, 2018, 256, 226-233.

243 H. Li, L. Chen, H. Wu, H. He and Y. Jin, Langmuir, 2014, 30, 15016-15021.

244 L. Zhang, Y. Han, J. Zhu, Y. Zhai and S. Dong, Anal. Chem., 2015, 87, 2033-2036.

245 X. Bao, Y. Yuan, J. Chen, B. Zhang, D. Li, D. Zhou, P. Jing, G. Xu, Y. Wang, K. Holá, D. Shen, C. Wu, L. Song, C. Liu, R. Zbořil and S. Qu, Light: Sci. Appl., 2018, 7, 91.

246 U. N. Pan, R. Khandelia, P. Sanpui, S. Das, A. Paul and A. Chattopadhyay, ACS Appl. Mater. Interfaces, 2017, 9, 19495-19501.

247 X. Cheng, R. Sun, L. Yin, Z. Chai, H. Shi and M. Gao, Adv. Mater., 2017, 29, 1604894. 
248 S. Kang, S. H. Bhang, S. Hwang, J. Yoon, J. Song, H. Jang, S. Kim and B. Kim, ACS Nano, 2015, 9(10), 9678-9690.

249 A. Espinosa, R. D. Corato, J. Kolosnjaj-Tabi, P. Flaud, T. Pellegrino and C. Wilhelm, ACS Nano, 2016, 10, 2436-2446.

250 A. Espinosa, A. Curcio, S. Cabana, G. Radtke, M. Bugnet, J. Kolosnjaj-Tabi, C. Péchoux, C. Alvarez-Lorenzo, G. A. Botton, A. K. A. Silva, A. Abou-Hassan and C. Wilhelm, ACS Nano, 2018, 12, 6523-6535.

251 J. Ge, Q. Jia, W. Liu, L. Guo, Q. Liu, M. Lan, H. Zhang, X. Meng and P. Wang, Adv. Mater., 2015, 27, 4169-4177.
252 M. Zheng, Y. Li, S. Liu, W. Wang, Z. Xie and X. Jing, ACS Appl. Mater. Interfaces, 2016, 8, 23533-23541.

253 M. Lan, L. Guo, S. Zhao, Z. Zhang, Q. Jia, L. Yan, J. Xia, H. Zhang, P. Wang and W. Zhang, Adv. Ther., 2018, 1, 1800077.

254 F. A. Permatasari, H. Fukazawa, T. Ogi, F. Iskandar and K. Okuyama, ACS Appl. Nano Mater., 2018, 1, 2368-2375.

255 B. Geng, D. Yang, D. Pan, L. Wang, F. Zheng, W. Shen, C. Zhang and X. Li, Carbon, 2018, 134, 153-162.

256 O. Neumann, A. S. Urban, J. Day, S. Lal, P. Nordlander and N. J. Halas, ACS Nano, 2013, 7(1), 42-49. 